\title{
Probing Catalyst Function - Electronic Modulation of Chiral Polyborate Anionic Catalysts.
}

Wynter, E. G. Osminski, ${ }^{\dagger}$ Zhenjie Lu, ${ }^{\dagger}$ Wenjun Zhao, ${ }^{\dagger}$ Aliakbar Mohammadlou, ${ }^{\dagger}$ Xiaopeng Yin, ${ }^{\dagger}$ Emily C. Matthews, ${ }^{\dagger}$ Virginia M. Canestraight, ${ }^{\dagger}$ Richard J. Staples, ${ }^{\dagger}$ Connor J. Allen, ${ }^{\dagger}$ Jennifer S. Hirschi* ${ }^{\dagger}{ }^{\dagger}$ and William D. Wulff* ${ }^{\dagger}$

${ }^{\dagger}$ Department of Chemistry, Michigan State University, East Lansing, MI 48824

Department of Chemistry, Binghamton University, Binghamton, New York 139802

wulff@chemistry.msu.edu

\section{Supporting Information}

Table of contents

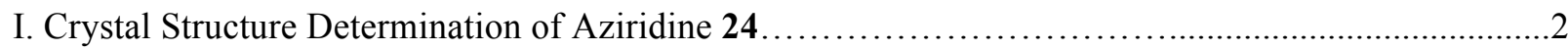

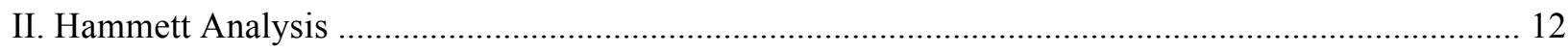

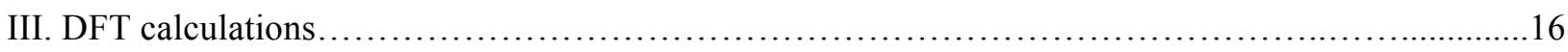

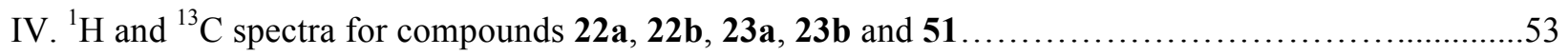


I. Crystal Structure Determination of aziridine 24.

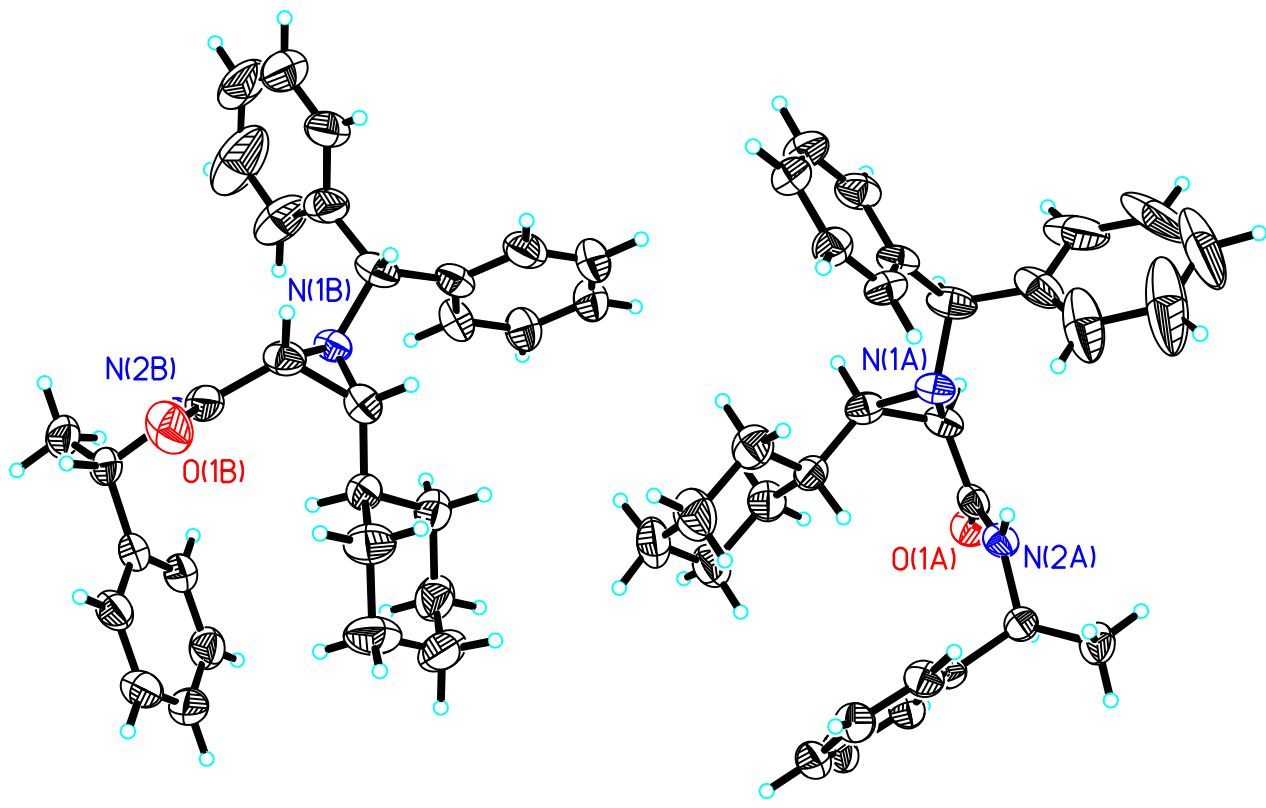


A colorless block crystal with dimensions $0.38 \times 0.29 \times 0.17 \mathrm{~mm}$ was mounted on a Nylon loop using very small amount of paratone oil.

Data were collected using a Bruker CCD (charge coupled device) based diffractometer equipped with an Oxford Cryostream low-temperature apparatus operating at $173 \mathrm{~K}$. Data were measured using omega and phi scans of $1.0^{\circ}$ per frame for $20 \mathrm{~s}$. The total number of images was based on results from the program $\operatorname{COSMO}^{1}$ where redundancy was expected to be 4.0 and completeness to $0.83 \AA$ to $100 \%$. Cell parameters were retrieved using APEX II software ${ }^{2}$ and refined using SAINT on all observed reflections. Data reduction was performed using the SAINT software ${ }^{3}$ which corrects for Lp. Scaling and absorption corrections were applied using SADABS ${ }^{4}$ multi-scan technique, supplied by George Sheldrick. The structures are solved by the direct method using the SHELXS-97 program and refined by least squares method on $\mathrm{F}^{2}$, SHELXL- 97, which are incorporated in SHELXTL-PC V $6.10 .^{5}$

The structure was solved in the space group $\mathrm{P} 2{ }_{1} 2_{1} 2_{1}(\# 19)$. All non-hydrogen atoms are refined anisotropically. Hydrogens were calculated by geometrical methods and refined as a riding model. The Flack ${ }^{6}$ parameter is used to determine chirality of the crystal studied, the value should be near zero, a value of one is the other enantiomer and a value of 0.5 is racemic. The Flack parameter was refined to $-0.1(3)$, confirming the absolute stereochemistryAll drawings are done at $50 \%$ ellipsoids.

\section{References}

1. COSMO V1.61, Software for the CCD Detector Systems for Determining Data Collection Parameters. Bruker Analytical X-ray Systems, Madison, WI (2009).

2. APEX2 V2010.11-3. Software for the CCD Detector System; Bruker Analytical X-ray Systems, Madison, WI (2010).

3. SAINT V 7.68A Software for the Integration of CCD Detector System Bruker Analytical X-ray Systems, Madison, WI (2010). 
4. SADABS V2008/2 Program for absorption corrections using Bruker-AXS CCD based on the method of Robert Blessing; Blessing, R.H. Acta Cryst. A51, 1995, 33-38.

5. Sheldrick, G.M. "A short history of SHELX". Acta Cryst. A64, 2008, 112-122.

6. Flack, H. D. Acta Cryst. A39, 1983, 876-881.

${ }^{\mathrm{a}}$ Obtained with graphite monochromated $\mathrm{Cu} \mathrm{K} \alpha(\lambda=1.54178 \AA \AA)$ radiation.

${ }^{\mathrm{b}} R 1=\Sigma|| \mathrm{F}_{\mathrm{o}}|-| \mathrm{F}_{\mathrm{c}} /\left|\mathrm{F}_{\mathrm{o}}\right| \quad{ }^{\mathrm{c}} W R_{2}=\left\{\mid\left[W\left(\mathrm{~F}_{\mathrm{o}}{ }^{2}-F_{\mathrm{c}}{ }^{2}\right)^{2} \cdot\left\{\Sigma\left[\mathrm{W}\left(\mathrm{F}_{\mathrm{o}}{ }^{2}\right)^{2}\right]\right\}^{1 / 2}\right.\right.$.

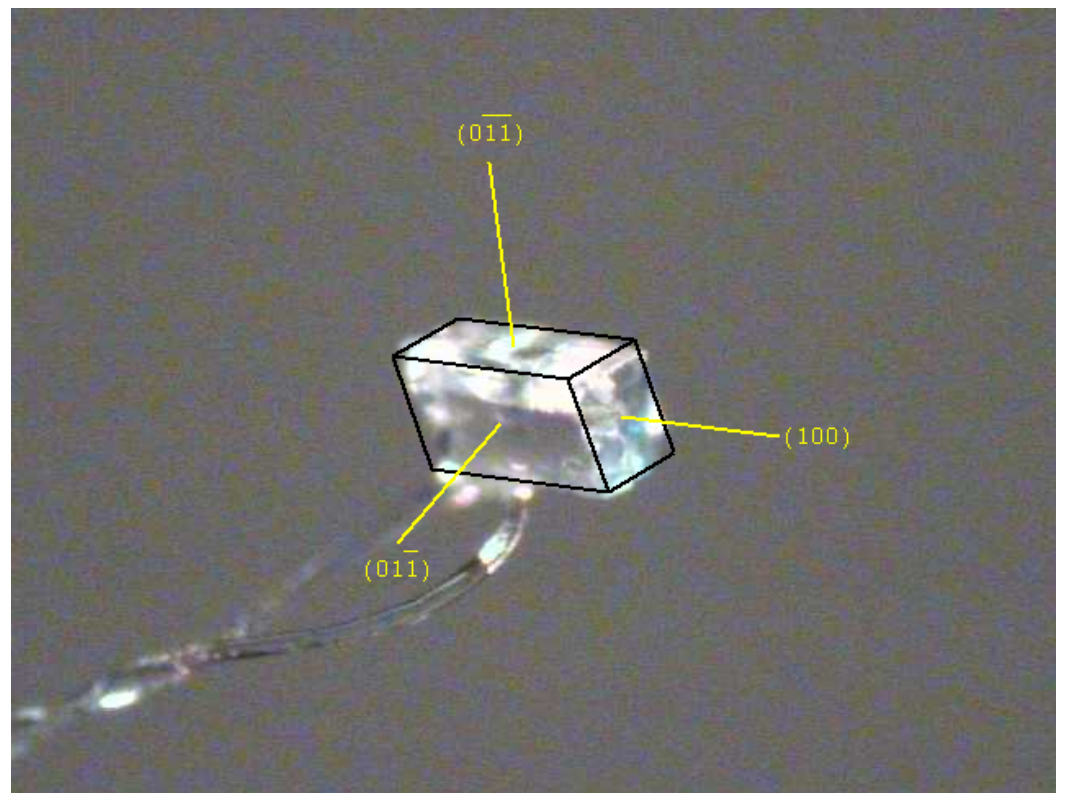

The following are $50 \%$ thermal ellipsoidal drawings of the molecule in the asymmetric cell with various amount of labeling. 


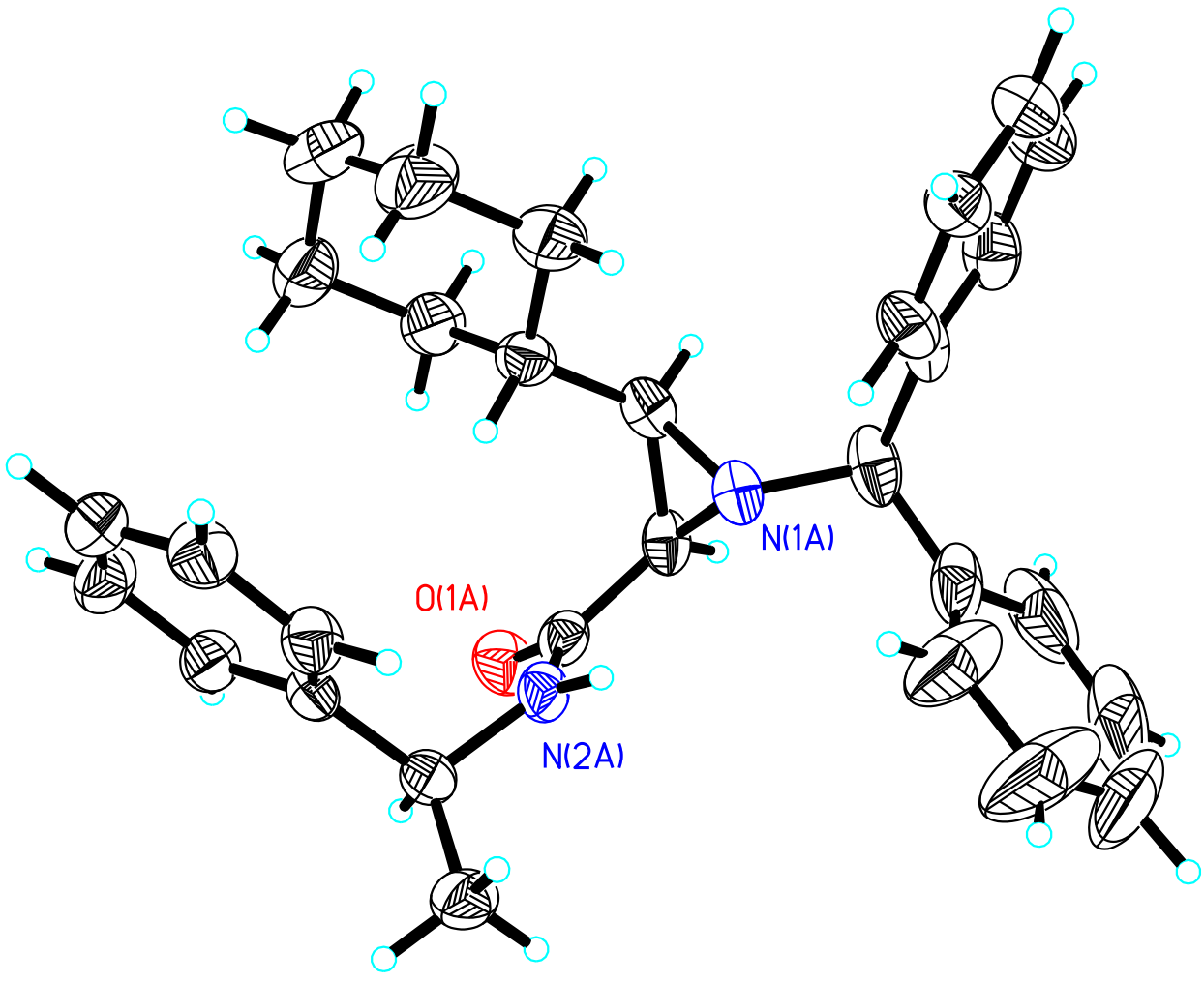




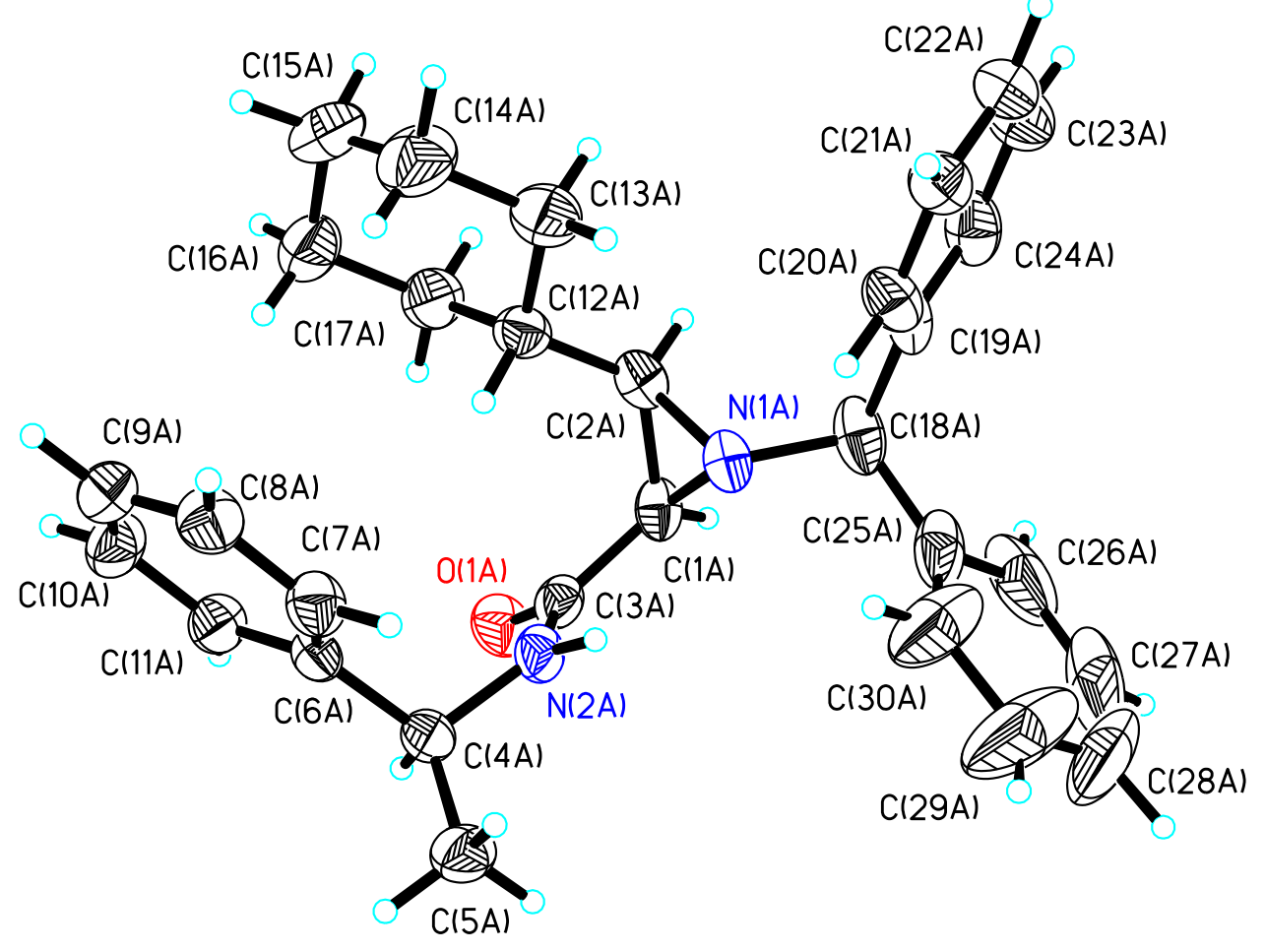




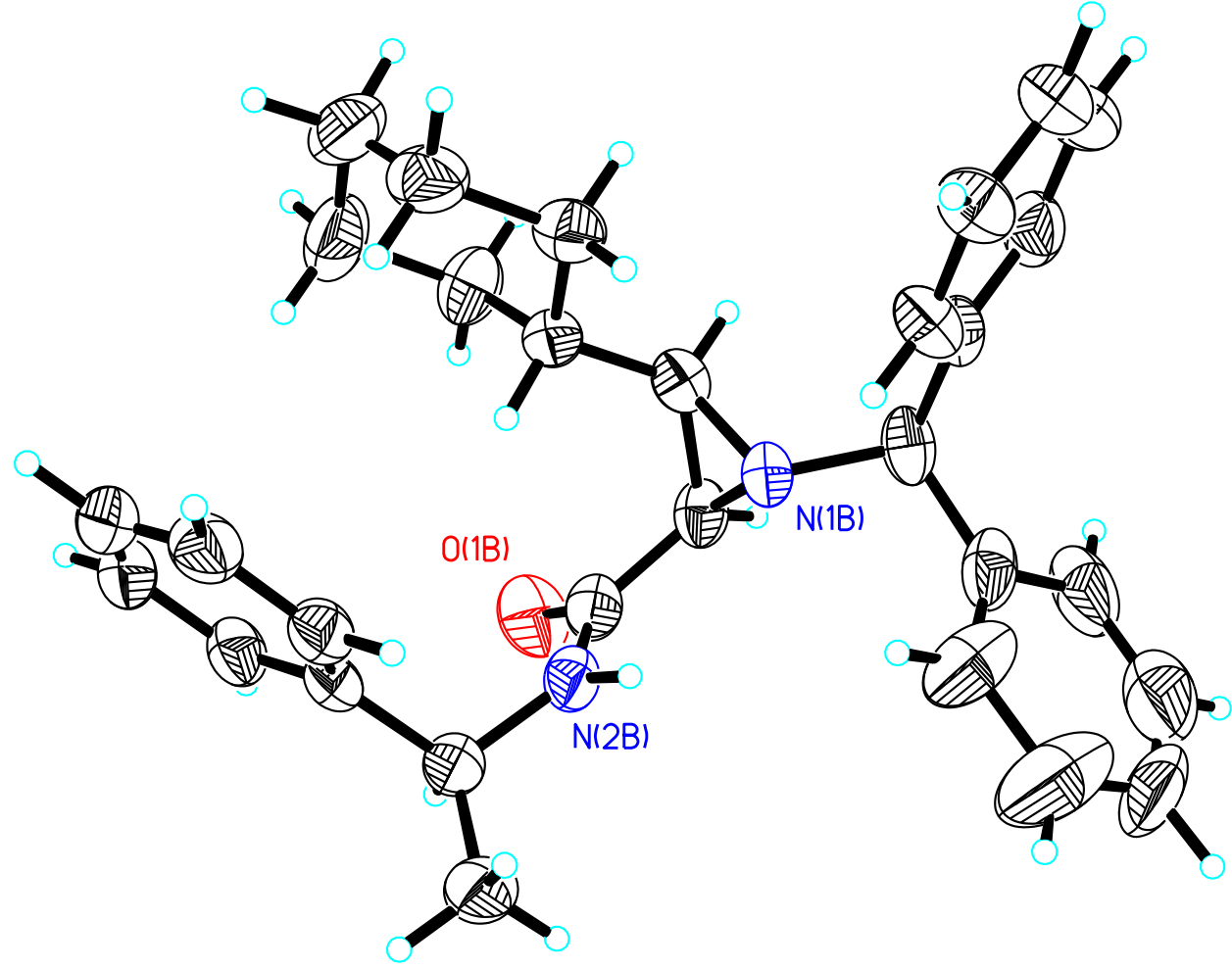




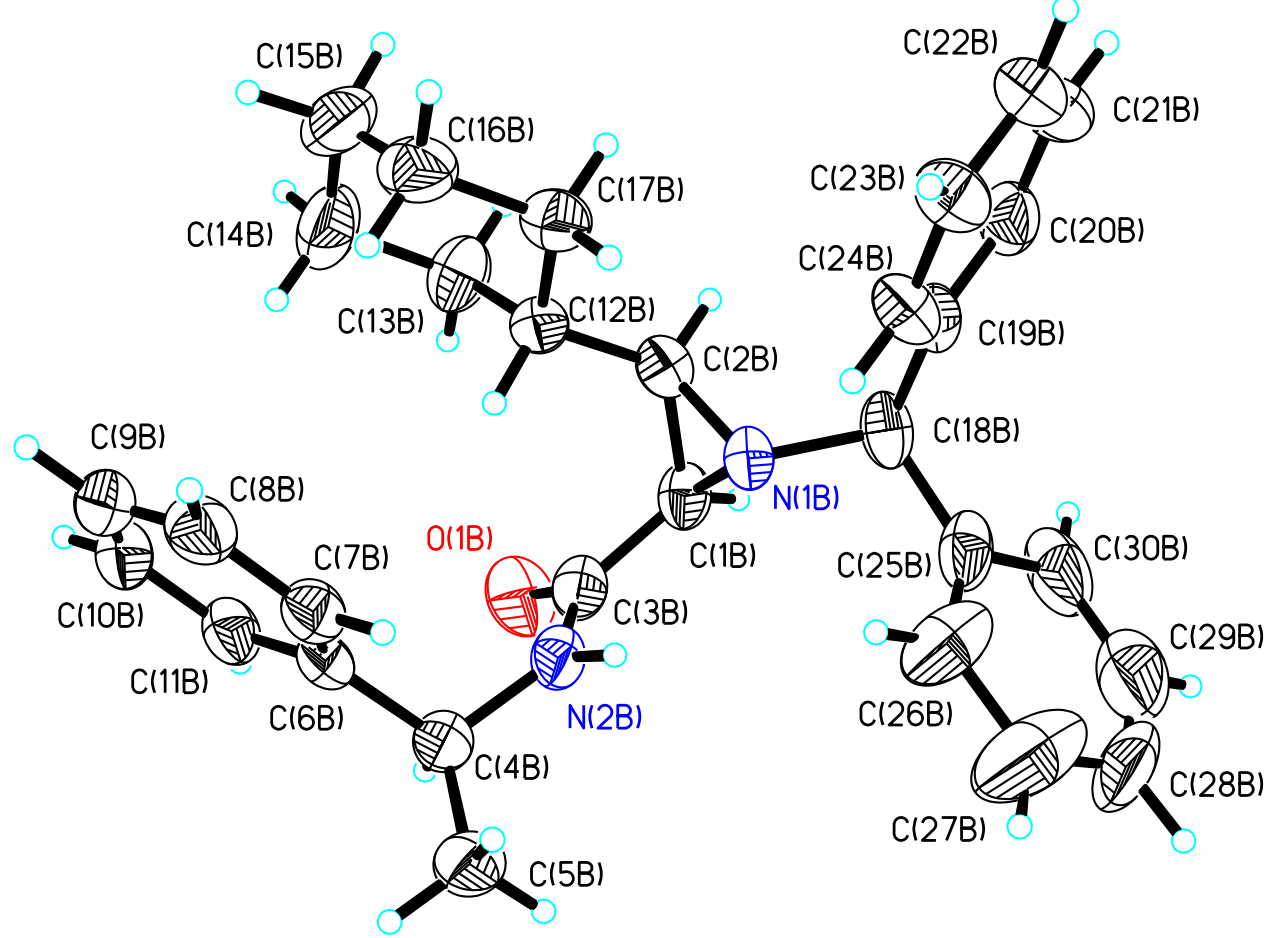




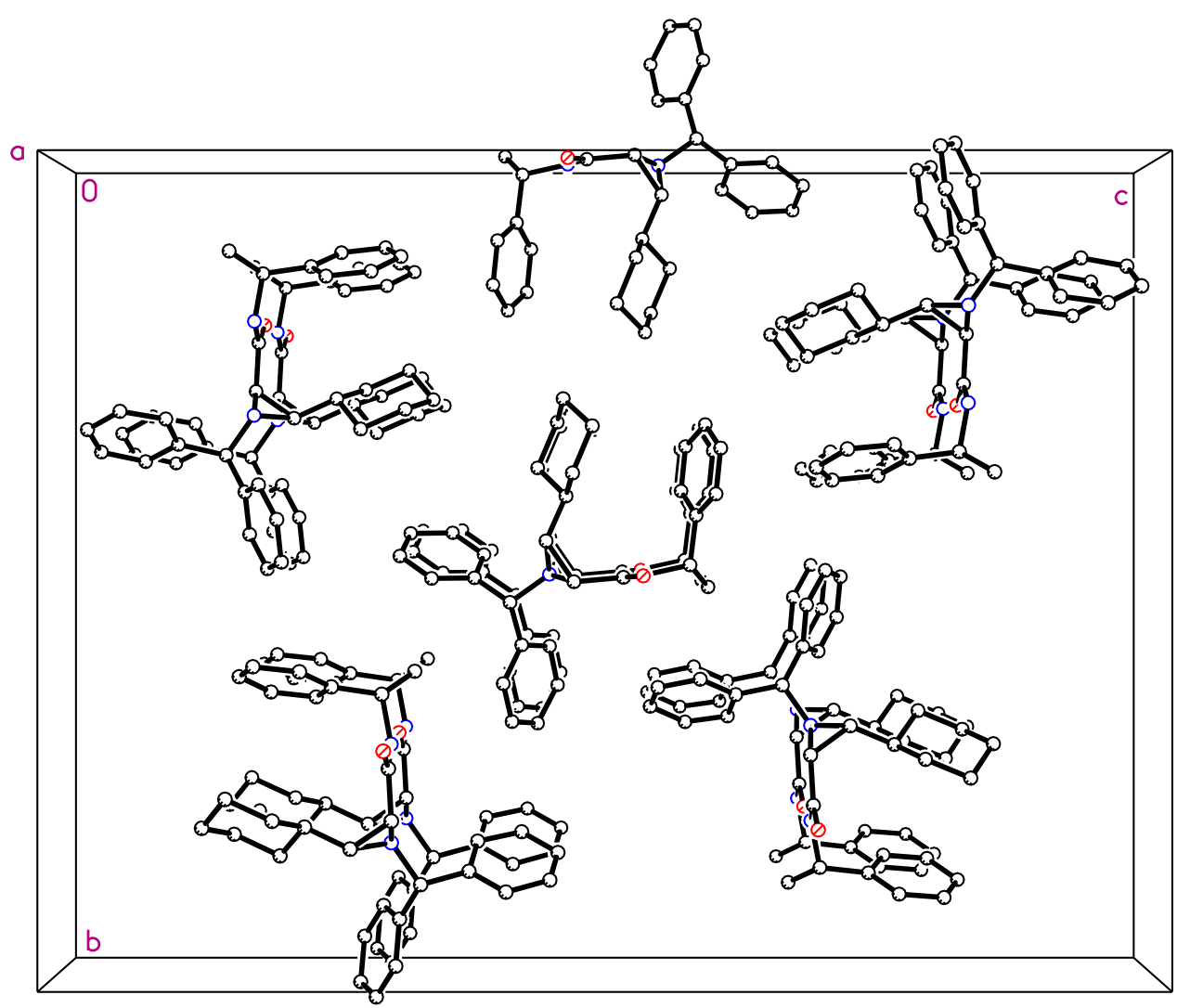

This is a drawing of the packing along the a-axis. 
Table SI-1. Crystal data and structure refinement for 24.

Identification code

Empirical formula

Formula weight

Temperature

Wavelength

Crystal system

Space group

Unit cell dimensions

Volume

Z

Density (calculated)

Absorption coefficient

$\mathrm{F}(000)$

Crystal size

Theta range for data collection

Index ranges

Reflections collected

Independent reflections

Completeness to theta $=67.88^{\circ}$

Absorption correction

Max. and min. transmission

Refinement method
24

C30 H34 N2 O

438.59

172(2) K

$1.54178 \AA$

Orthorhombic

P 212121

$\mathrm{a}=6.09190(10) \AA$

$\alpha=90^{\circ}$.

$\mathrm{b}=24.9451(2) \AA$

$\beta=90^{\circ}$.

$c=33.6623(3) \AA$

$\gamma=90^{\circ}$

5115.43(10) $\AA^{3}$

8

$1.139 \mathrm{Mg} / \mathrm{m}^{3}$

$0.527 \mathrm{~mm}^{-1}$

1888

$0.38 \times 0.29 \times 0.17 \mathrm{~mm}^{3}$

2.20 to $67.88^{\circ}$.

$-7<=\mathrm{h}<=7,-29<=\mathrm{k}<=29,-39<=\mathrm{l}<=40$

33702

$9133[\mathrm{R}($ int $)=0.0235]$

$99.3 \%$

Semi-empirical from equivalents

0.9167 and 0.8241

Full-matrix least-squares on $\mathrm{F}^{2}$ 
Data / restraints / parameters

Goodness-of-fit on $\mathrm{F}^{2}$

Final $\mathrm{R}$ indices $[\mathrm{I}>2 \operatorname{sigma}(\mathrm{I})]$

$\mathrm{R}$ indices (all data)

Absolute structure parameter

Largest diff. peak and hole
9133 / 0 / 598

0.983

$\mathrm{R} 1=0.0527, \mathrm{wR} 2=0.1427$

$\mathrm{R} 1=0.0544, \mathrm{wR} 2=0.1448$

$-0.1(3)$

0.430 and -0.353 e. $\AA^{-3}$ 


\section{Hammett Analysis.}

All of the data points collected for the Hammett analysis represent reactions that went to $>95 \%$ completion. While some data points represent the average for reactions run more than once, most of the data points are from reactions with a single run.

A. Various BOROX Catalysts in the Aziridination of the Phenyl Substituted Imine 22a.
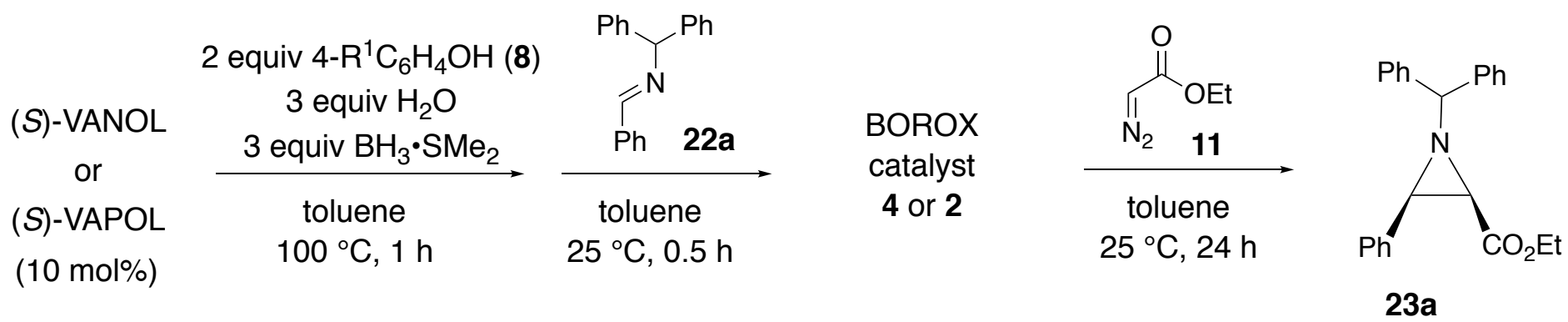

With the VANOL Ligand
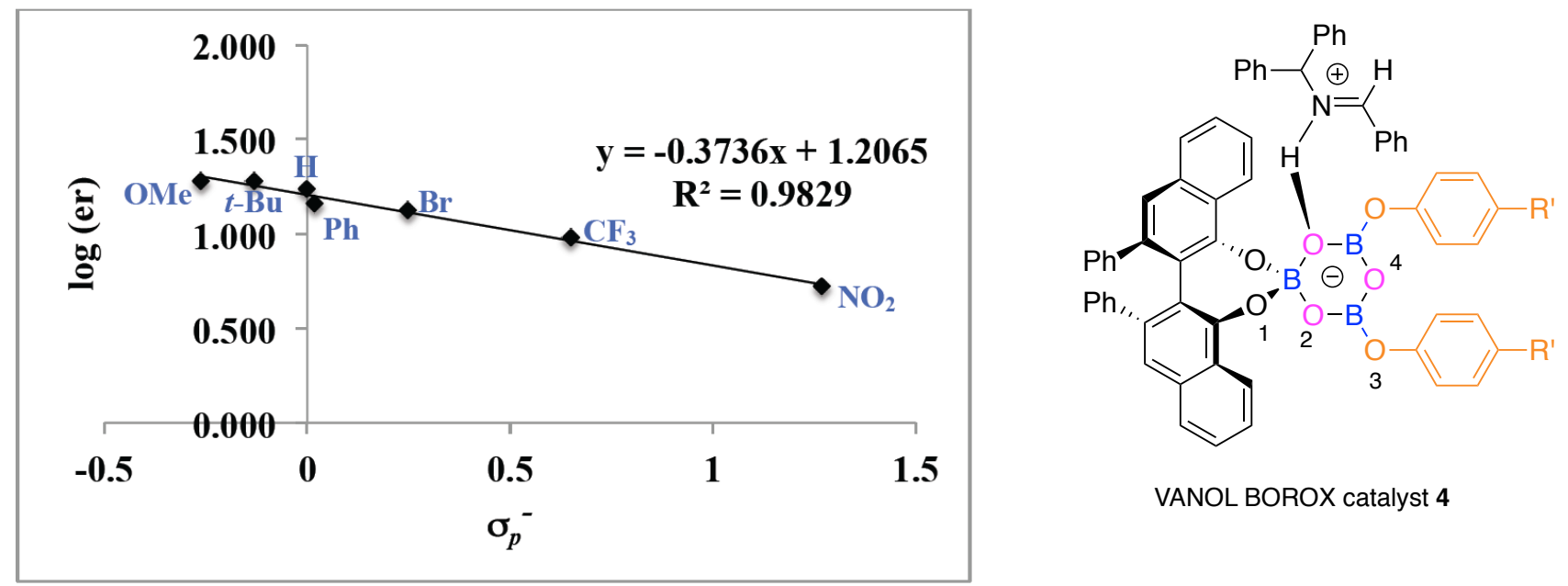

VANOL BOROX catalyst 4 


\begin{tabular}{ccccc}
\hline $\mathrm{R}^{1}$ & \% ee & er & log er & $\sigma_{\mathrm{p}}{ }^{-}$ \\
\hline $\mathrm{MeO}$ & 90 & $95: 5$ & 1.279 & -0.26 \\
$\mathrm{Me}_{3} \mathrm{C}$ & 90 & $95: 5$ & 1.279 & -0.13 \\
$\mathrm{H}$ & 89 & $94.5: 5.5$ & 1.235 & 0 \\
$\mathrm{C}_{6} \mathrm{H}_{5}$ & 87 & $93.5: 6.5$ & 1.158 & 0.02 \\
$\mathrm{Br}$ & 86 & $93: 7$ & 1.123 & 0.25 \\
$\mathrm{CF}_{3}$ & 81 & $90.5: 9.5$ & 0.979 & 0.65 \\
$\mathrm{NO}_{2}$ & 68 & $84: 16$ & 0.720 & 1.27 \\
\hline
\end{tabular}

With the VAPOL Ligand
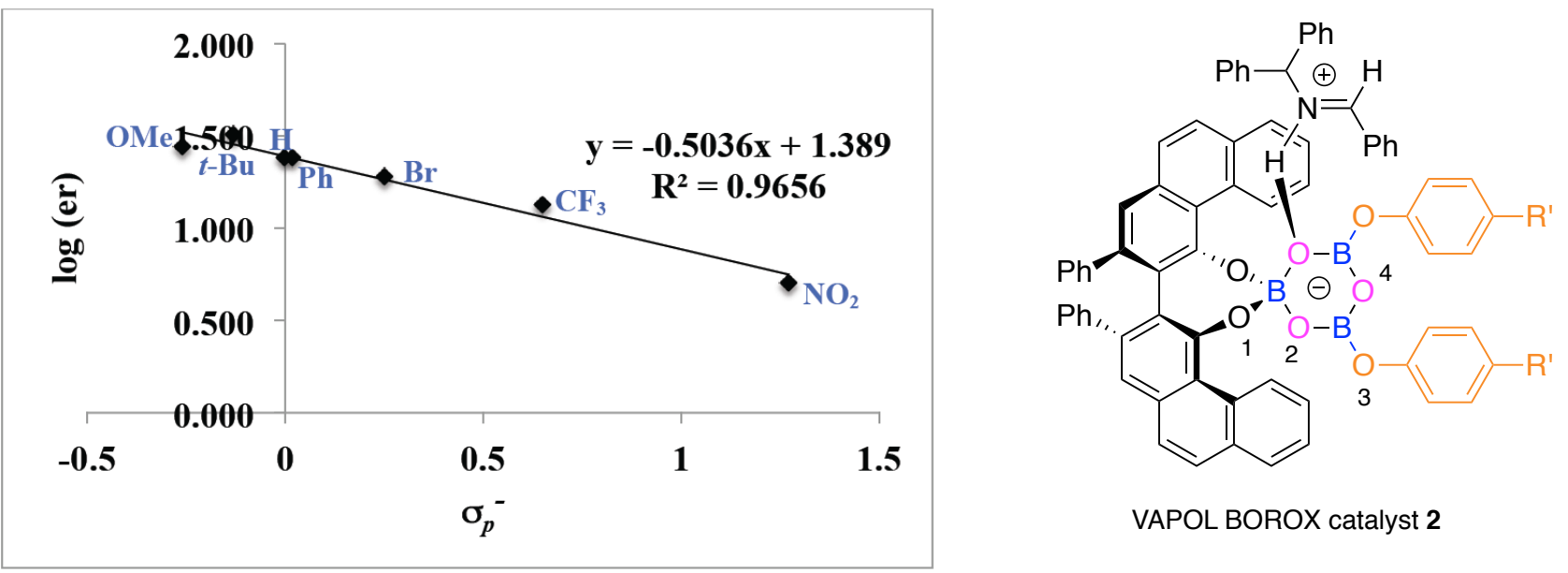

VAPOL BOROX catalyst 2

\begin{tabular}{ccccc}
\hline $\mathrm{R}^{3}$ & $\%$ ee & er & log er & $\sigma_{\mathrm{p}}{ }^{-}$ \\
\hline $\mathrm{MeO}$ & 93 & $96.5: 3.5$ & 1.440 & -0.26 \\
$\mathrm{Me}_{3} \mathrm{C}$ & 94 & $97: 3$ & 1.510 & -0.13 \\
$\mathrm{H}$ & 92 & $96: 4$ & 1.380 & 0 \\
$\mathrm{C}_{6} \mathrm{H}_{5}$ & 92 & $96: 4$ & 1.380 & 0.02 \\
$\mathrm{Br}$ & 90 & $95: 5$ & 1.279 & 0.25 \\
$\mathrm{CF}_{3}$ & 86 & $93: 7$ & 1.123 & 0.65 \\
$\mathrm{NO}_{2}$ & 67 & $83.5: 16.5$ & 0.704 & 1.27 \\
\hline
\end{tabular}


B. Various BOROX Catalysts in the Aziridination of the Cyclohexyl Substituted Imine $\mathbf{2 2 b .}$
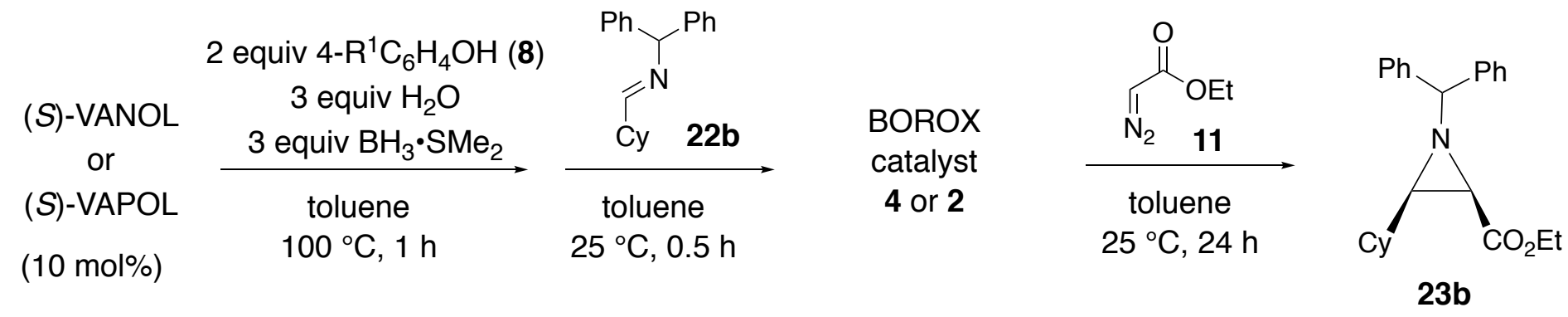

With the VANOL Ligand
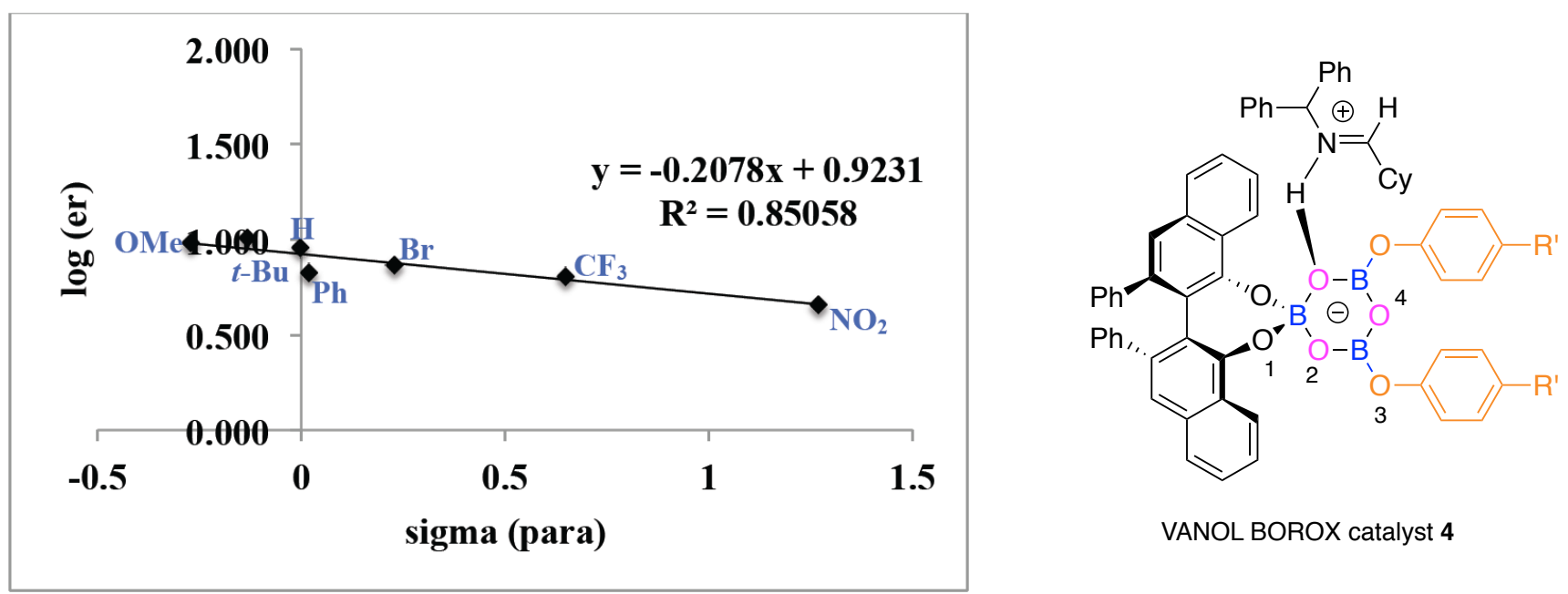

VANOL BOROX catalyst 4

\begin{tabular}{ccccc}
\hline $\mathrm{R}^{1}$ & $\%$ ee & er & log er & $\sigma_{\mathrm{p}}^{-}$ \\
\hline $\mathrm{MeO}$ & 81 & $90.5: 9.5$ & 0.979 & -0.26 \\
$\mathrm{Me}_{3} \mathrm{C}$ & 82 & $91: 9$ & 1.005 & -0.13 \\
$\mathrm{H}$ & 79 & $89.5: 10.5$ & 0.931 & 0 \\
$\mathrm{C}_{6} \mathrm{H}_{5}$ & 74 & $87: 13$ & 0.826 & 0.02 \\
$\mathrm{Br}$ & 80 & $90: 10$ & 0.954 & 0.25 \\
$\mathrm{CF}_{3}$ & 73 & $86.5: 13.5$ & 0.807 & 0.65 \\
$\mathrm{NO}_{2}$ & 64 & $82: 18$ & 0.659 & 1.27 \\
\hline
\end{tabular}


With the VAPOL Ligand
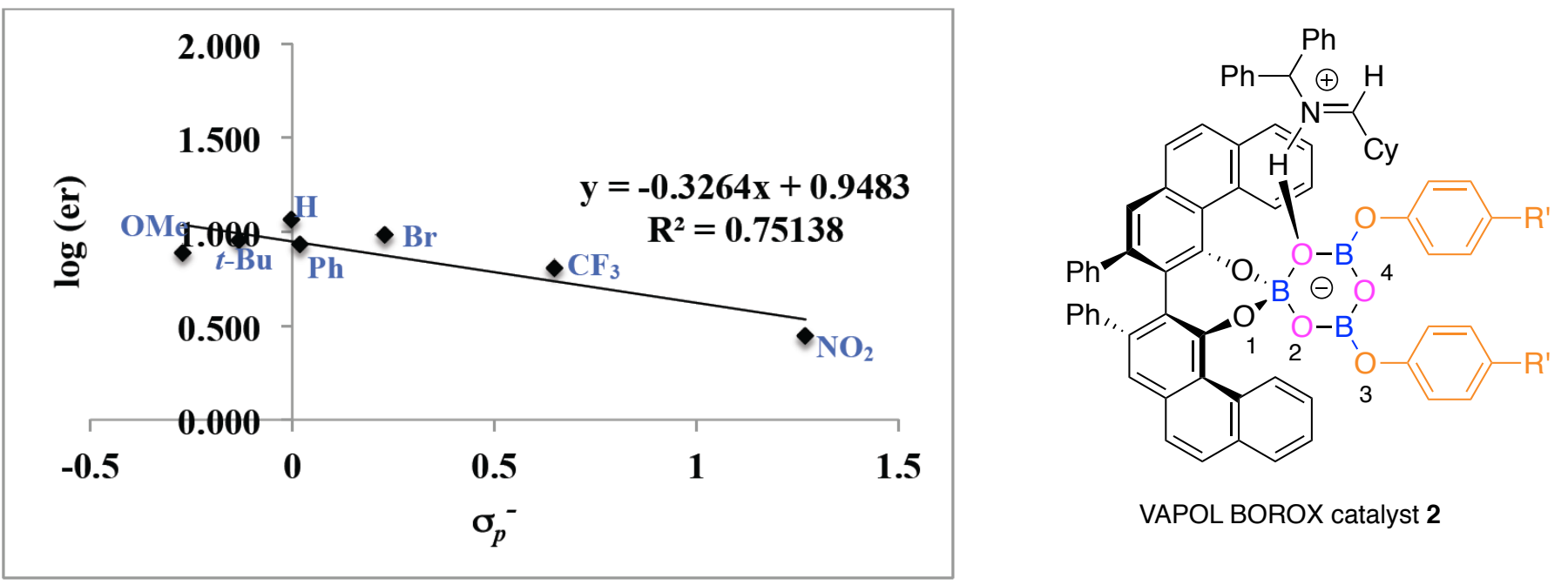

\begin{tabular}{ccccc}
\hline $\mathrm{R}^{3}$ & $\%$ ee & er & log er & $\sigma_{\mathrm{p}}{ }^{-}$ \\
\hline $\mathrm{MeO}$ & 77 & $88.5: 11.5$ & 0.886 & -0.26 \\
$\mathrm{Me}_{3} \mathrm{C}$ & 80 & $90: 10$ & 0.954 & -0.13 \\
$\mathrm{H}$ & 74 & $87: 13$ & 0.826 & 0 \\
$\mathrm{C}_{6} \mathrm{H}_{5}$ & 79 & $89.5: 10.5$ & 0.931 & 0.02 \\
$\mathrm{Br}$ & 76 & $88: 12$ & 0.865 & 0.25 \\
$\mathrm{CF}_{3}$ & 73 & $86.5: 13.5$ & 0.807 & 0.65 \\
$\mathrm{NO}_{2}$ & 67 & $83.5: 16.5$ & 0.704 & 1.27 \\
\hline
\end{tabular}




\section{DFT Calculations}

\section{Catalyst Structures Used for ADCH Charge Analysis:}

Optimizations of catalyst-borox complexes were carried out at the M06-2X/6-31G* level of theory as implemented by the Gaussian 16 software package with bulk solvent effects accounted for by the self-consistent reaction field polarizable continuum model (PCM) as implemented for toluene.

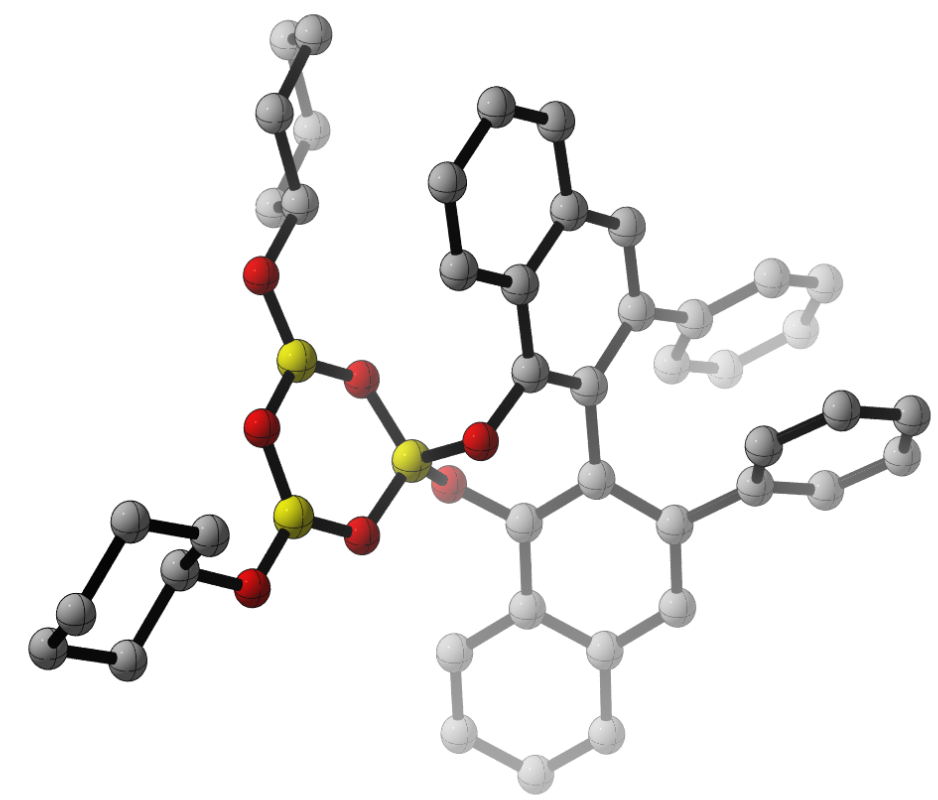

Entry 1

Filename: cat1

RRHO Thermochemistry $(T=298)$

Zero-point correction $(\mathrm{RRHO})=$

Thermal correction to Energy $(\mathrm{RRHO})=$

Thermal correction to Enthalpy (RRHO)=

0.794775 (Hartree/Particle)

0.837485

0.838429

Thermal correction to Gibbs Free Energy $(\mathrm{RRHO})=$

Sum of electronic and zero-point Energies $(\mathrm{RRHO})=$

Sum of electronic and thermal Energies $(\mathrm{RRHO})=$

0.718671

Sum of electronic and thermal Enthalpies (RRHO)=

$-2302.086172$

$-2302.043462$

Sum of electronic and thermal Free Energies $(\mathrm{RRHO})=\quad-2302.162276$

Quasi-RRHO Thermochemistry ( $T=298$, v0 $=100 \mathrm{~cm}-1)$

Zero-point correction (QRRHO)=

0.794775 (Hartree/Particle) 
Thermal correction to Energy $(\mathrm{QRRHO})=\quad 0.837485$

Thermal correction to Enthalpy (QRRHO)= 0.838429

Thermal correction to Gibbs Free Energy $(\mathrm{QRRHO})=\quad 0.727905$

Sum of electronic and zero-point Energies (QRRHO)=

$-2302.086172$

Sum of electronic and thermal Energies $(\mathrm{QRRHO})=\quad-2302.043462$

Sum of electronic and thermal Enthalpies $(\mathrm{QRRHO})=\quad-2302.042518$

Sum of electronic and thermal Free Energies (QRRHO)= $\quad-2302.153042$

Quasi-harmonic Thermochemistry $(T=298$, cutoff $=100 \mathrm{~cm}-1)$

Zero-point correction $(\mathrm{QHO})=$

Thermal correction to Energy $(\mathrm{QHO})=$

0.794775 (Hartree/Particle)

Thermal correction to Enthalpy $(\mathrm{QHO})=$

0.837673

0.838429

Thermal correction to Gibbs Free Energy $(\mathrm{QHO})=$

0.729412

Sum of electronic and zero-point Energies (QHO)=

$-2302.086172$

Sum of electronic and thermal Energies $(\mathrm{QHO})=$

$-2302.043274$

Sum of electronic and thermal Enthalpies $(\mathrm{QHO})=$

$-2302.042518$

Sum of electronic and thermal Free Energies $(\mathrm{QHO})=$

$-2302.151535$

$\begin{array}{lrrr}\text { C } & 4.2345540 & 0.4294230 & -1.4977680 \\ \text { C } & 4.0414770 & 1.1147040 & -0.2918330 \\ \text { C } & 5.1570700 & 1.6623690 & 0.3521640 \\ \text { C } & 6.4322490 & 1.5243470 & -0.1860390 \\ \text { C } & 6.6146640 & 0.8333340 & -1.3814020 \\ \text { C } & 5.5101840 & 0.2892690 & -2.0355200 \\ \text { C } & 2.6848510 & 1.2977990 & 0.2918090 \\ \text { C } & 1.7239110 & 0.2335620 & 0.3514290 \\ \text { C } & 0.4491890 & 0.5090440 & 0.8274830 \\ \text { C } & 0.0959380 & 1.8076230 & 1.3065830 \\ \text { C } & 1.0605310 & 2.8452850 & 1.2580860 \\ \text { C } & 2.3470490 & 2.5568680 & 0.7400270 \\ \text { C } & 0.6835190 & 4.1448530 & 1.6916230 \\ \text { C } & -0.5834460 & 4.3880950 & 2.1627390 \\ \text { C } & -1.5354670 & 3.3405520 & 2.2298800 \\ \text { C } & -1.2031860 & 2.0774480 & 1.8058700 \\ \text { C } & 1.9975870 & -1.1687090 & -0.0724380 \\ \text { C } & 1.0999560 & -1.7723810 & -0.9494080 \\ \text { C } & 1.3295110 & -3.0985480 & -1.4387000 \\ \text { C } & 2.4897250 & -3.8012230 & -1.0267020 \\ \text { C } & 3.3686140 & -3.1856410 & -0.1028980 \\ \text { C } & 3.1349290 & -1.9177270 & 0.3818020 \\ \text { C } & 0.4160180 & -3.7170720 & -2.3285630 \\ \text { C } & 0.6556430 & -4.9847770 & -2.7990880 \\ \text { C } & 1.8174870 & -5.6881380 & -2.3977660 \\ \text { C } & 2.7099470 & -5.1110730 & -1.5294420 \\ \text { O } & 0.0297430 & -1.1234590 & -1.4290570 \\ \text { B } & -1.0550540 & -0.7095790 & -0.5223630 \\ \text { O } & -0.4955310 & -0.4456540 & 0.8345540 \\ \text { C } & 4.0788190 & -1.3882150 & 1.4039560 \\ \text { C } & 3.6221220 & -0.7580360 & 2.5682040 \\ \text { C } & 4.5223640 & -0.2881580 & 3.5194640 \\ \text { C } & 5.8944030 & -0.4455080 & 3.3291220 \\ \text { C } & 6.3588970 & -1.0815940 & 2.1805080\end{array}$

$\begin{array}{rrrr}\text { C } & 5.4584960 & -1.5469740 & 1.2280170 \\ \mathrm{O} & -2.0573240 & -1.7520770 & -0.3733550 \\ \mathrm{~B} & -3.3381750 & -1.4025860 & -0.1434480 \\ \mathrm{O} & -3.7773970 & -0.0855470 & -0.2435990 \\ \mathrm{~B} & -2.8871420 & 0.8558490 & -0.7466630 \\ \mathrm{O} & -3.3749540 & 2.1303430 & -0.8997160 \\ \mathrm{O} & -4.2493350 & -2.3783220 & 0.1865560 \\ \mathrm{O} & -1.6197930 & 0.5259120 & -1.0647090 \\ \mathrm{H} & -0.0498490 & -5.4562710 & -3.4767570 \\ \mathrm{H} & -1.9193960 & 1.2622710 & 1.8429910 \\ \mathrm{H} & -0.8602160 & 5.3869970 & 2.4883730 \\ \mathrm{H} & 1.4179570 & 4.9453220 & 1.6404150 \\ \mathrm{H} & 3.0677350 & 3.3661270 & 0.6522730 \\ \mathrm{H} & 5.0209590 & 2.1624080 & 1.3072800 \\ \mathrm{H} & 7.2866500 & 1.9415920 & 0.3395300 \\ \mathrm{H} & 7.6098190 & 0.7177490 & -1.8010230 \\ \mathrm{H} & 5.6406250 & -0.2448270 & -2.9722530 \\ \mathrm{H} & 3.3773170 & 0.0060870 & -2.0124790 \\ \mathrm{H} & 5.8231490 & -2.0010060 & 0.3105730 \\ \mathrm{H} & 7.4262260 & -1.1988600 & 2.0149510 \\ \mathrm{H} & 6.5951930 & -0.0741800 & 4.0713190 \\ \mathrm{H} & 4.1491090 & 0.1982530 & 4.4160330 \\ \mathrm{H} & 2.5543150 & -0.6372170 & 2.7233850 \\ \mathrm{H} & 4.2189420 & -3.7524650 & 0.2677440 \\ \mathrm{H} & 3.5993470 & -5.6489630 & -1.2096140 \\ \mathrm{H} & 1.9964270 & -6.6909260 & -2.7753290 \\ \mathrm{H} & -0.4715880 & -3.1637180 & -2.6133110 \\ \mathrm{H} & -2.5327880 & 3.5430240 & 2.6091360 \\ \mathrm{C} & -5.5902720 & -2.0347100 & 0.4899680 \\ \mathrm{C} & -6.4499480 & -3.2795090 & 0.2973010 \\ \mathrm{C} & -5.6997630 & -1.5147490 & 1.9223430 \\ \mathrm{H} & -5.9363210 & -1.2463080 & -0.1946460 \\ \mathrm{C} & -7.9095280 & -3.0077820 & 0.6701420 \\ \mathrm{H} & -6.0422620 & -4.0759360 & 0.9350270\end{array}$




$\begin{array}{lrrrrrrr}\mathrm{H} & -6.3631810 & -3.6214490 & -0.7398750 & \mathrm{H} & -1.5010710 & 2.9922610 & -0.7928080 \\ \mathrm{C} & -7.1570420 & -1.2376190 & 2.3005740 & \mathrm{C} & -1.3157600 & 4.2774950 & -3.2247700 \\ \mathrm{H} & -5.2751880 & -2.2760740 & 2.5914150 & \mathrm{H} & -3.2181460 & 3.2361450 & -3.2969160 \\ \mathrm{H} & -5.0900380 & -0.6106130 & 2.0221280 & \mathrm{H} & -1.8216780 & 2.1640150 & -3.0823280 \\ \mathrm{C} & -8.0236170 & -2.4846090 & 2.1048320 & \mathrm{C} & -2.1111010 & 5.6392670 & -1.2542570 \\ \mathrm{H} & -8.5070720 & -3.9175520 & 0.5444990 & \mathrm{H} & -4.0274810 & 4.6218870 & -1.3049810 \\ \mathrm{H} & -8.3258630 & -2.2583870 & -0.0177090 & \mathrm{H} & -3.1830010 & 4.4762080 & 0.2429200 \\ \mathrm{H} & -7.2187030 & -0.8859230 & 3.3363400 & \mathrm{C} & -1.8633400 & 5.6320000 & -2.7658170 \\ \mathrm{H} & -7.5460780 & -0.4272720 & 1.6683250 & \mathrm{H} & -1.1752400 & 4.2688110 & -4.3114760 \\ \mathrm{H} & -9.0695890 & -2.2664940 & 2.3492660 & \mathrm{H} & -0.3254000 & 4.1190250 & -2.7760740 \\ \mathrm{H} & -7.6888950 & -3.2673320 & 2.7999350 & \mathrm{H} & -2.5323970 & 6.6009110 & -0.9393910 \\ \mathrm{C} & -2.4679950 & 3.1539420 & -1.2914660 & \mathrm{H} & -1.1518690 & 5.5239830 & -0.7296910 \\ \mathrm{C} & -2.2420830 & 3.1343380 & -2.8008260 & \mathrm{H} & -1.1732320 & 6.4372140 & -3.0435020 \\ \mathrm{C} & -3.0382910 & 4.4932740 & -0.8428320 & \mathrm{H} & -2.8118370 & 5.8306820 & -3.2850600\end{array}$

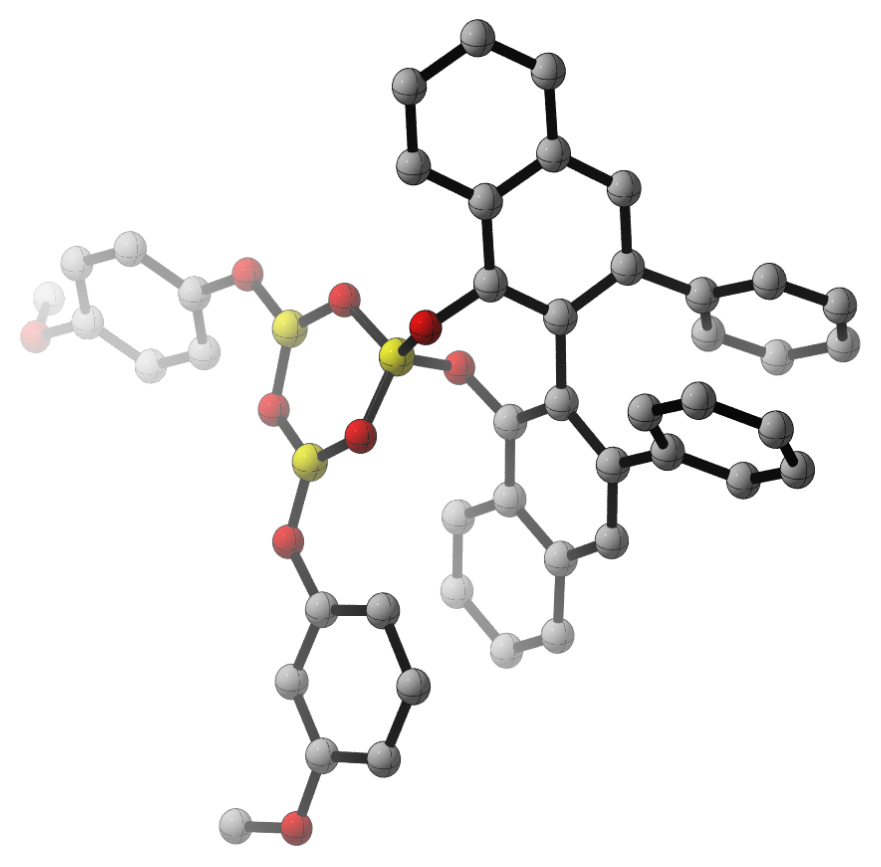

Entry 2

File Name: cat2

RRHO Thermochemistry $(T=298)$

Zero-point correction $(\mathrm{RRHO})=$

Thermal correction to Energy $(\mathrm{RRHO})=$

0.717966 (Hartree/Particle)

Thermal correction to Enthalpy $(\mathrm{RRHO})=$

0.763649

0.764593

Thermal correction to Gibbs Free Energy $(\mathrm{RRHO})=0.635302$

Sum of electronic and zero-point Energies $(\mathrm{RRHO})=\quad-2523.887478$ 
Sum of electronic and thermal Energies $(\mathrm{RRHO})=$

Sum of electronic and thermal Enthalpies $(\mathrm{RRHO})=$

Sum of electronic and thermal Free Energies $(\mathrm{RRHO})=$
$-2523.841795$

$-2523.840851$

$-2523.970142$

Quasi-RRHO Thermochemistry ( $T=298, v 0=100 \mathrm{~cm}-1)$

Zero-point correction $(\mathrm{QRRHO})=$

Thermal correction to Energy $(\mathrm{QRRHO})=$

Thermal correction to Enthalpy (QRRHO)=

Thermal correction to Gibbs Free Energy $(\mathrm{QRRHO})=$

Sum of electronic and zero-point Energies $(\mathrm{QRRHO})=$

Sum of electronic and thermal Energies (QRRHO)=

Sum of electronic and thermal Enthalpies $(\mathrm{QRRHO})=$

Sum of electronic and thermal Free Energies $(\mathrm{QRRHO})=$
0.717966 (Hartree/Particle)

0.763649

0.764593

0.646839

$-2523.887478$

$-2523.841795$

$-2523.840851$

$-2523.958605$

Quasi-harmonic Thermochemistry $(T=298$, cutoff $=100 \mathrm{~cm}-1)$

Zero-point correction $(\mathrm{QHO})=$

Thermal correction to Energy $(\mathrm{QHO})=$

Thermal correction to Enthalpy $(\mathrm{QHO})=$

Thermal correction to Gibbs Free Energy $(\mathrm{QHO})=$

Sum of electronic and zero-point Energies $(\mathrm{QHO})=$

Sum of electronic and thermal Energies $(\mathrm{QHO})=$

Sum of electronic and thermal Enthalpies $(\mathrm{QHO})=$

Sum of electronic and thermal Free Energies $(\mathrm{QHO})=$

$\begin{array}{lrrr}\text { C } & 4.6530830 & 0.5025010 & -1.4921550 \\ \text { C } & 4.3815870 & 1.1998340 & -0.3084300 \\ \text { C } & 5.4376280 & 1.8457750 & 0.3445780 \\ \text { C } & 6.7316740 & 1.7898370 & -0.1621060 \\ \text { C } & 6.9933690 & 1.0835210 & -1.3335630 \\ \text { C } & 5.9481760 & 0.4428140 & -1.9974980 \\ \text { C } & 3.0031500 & 1.2901550 & 0.2439920 \\ \text { C } & 2.1253900 & 0.1554020 & 0.3055750 \\ \text { C } & 0.8218900 & 0.3439310 & 0.7420110 \\ \text { C } & 0.3564440 & 1.6184050 & 1.1868850 \\ \text { C } & 1.2426030 & 2.7236940 & 1.1494550 \\ \text { C } & 2.5593280 & 2.5252060 & 0.6641940 \\ \text { C } & 0.7589520 & 3.9979190 & 1.5471000 \\ \text { C } & -0.5403210 & 4.1581980 & 1.9596890 \\ \text { C } & -1.4178600 & 3.0481610 & 2.0040410 \\ \text { C } & -0.9772200 & 1.8029290 & 1.6293310 \\ \text { C } & 2.5249430 & -1.2331050 & -0.0628050 \\ \text { C } & 1.7094630 & -1.9416960 & -0.9395830 \\ \text { C } & 2.0571560 & -3.2624670 & -1.3681360 \\ \text { C } & 3.2587330 & -3.8500200 & -0.8989160 \\ \text { C } & 4.0578040 & -3.1282010 & 0.0201660 \\ \text { C } & 3.7064940 & -1.8679380 & 0.4503620 \\ \text { C } & 1.2199810 & -3.9899650 & -2.2506900\end{array}$

0.717966 (Hartree/Particle)

0.763859

0.764593

0.649624

$-2523.887478$

$-2523.841585$

$-2523.840851$

$-2523.955820$
$-5.2509060$

$-5.8380820$

$-5.1551850$

$-1.3997990$

$-1.0131840$

$-0.6795540$

$-1.2280010$

$-0.5999000$

$-0.0309720$

$-0.0831800$

$-0.7140470$

$-1.2793200$

$-2.0937890$

$-1.7782470$

$-0.4872370$

0.4764220

1.7236160

2.9168410

3.0725410

4.3652280

5.4816010

5.3069470

4.0299390
$-2.6624920$

$-2.2064980$

$-1.3425310$

$-1.4800050$

$-0.6325020$

0.7347770

1.4786510

2.6065530

3.5681460

3.4235200

2.3102990

1.3478750

$-0.4987300$

$-0.3173520$

$-0.4438860$

$-0.9387550$

$-1.0945020$

$-1.3188760$

$-1.5735500$

$-1.7461090$

$-1.6877070$

$-1.4430900$

$-1.2589360$ 


$\begin{array}{rrrrrrrr}\mathrm{O} & -3.6683550 & -2.8052080 & -0.0111990 & \mathrm{H} & 8.0041530 & 1.0318560 & -1.7277920 \\ \mathrm{C} & -4.9898900 & -2.6417130 & 0.2796460 & \mathrm{H} & 6.1400630 & -0.1028400 & -2.9167610 \\ \mathrm{C} & -5.8873530 & -3.5663340 & -0.2556450 & \mathrm{H} & 3.8416950 & 0.0058320 & -2.0155900 \\ \mathrm{C} & -7.2402050 & -3.4922270 & 0.0585230 & \mathrm{H} & 6.3935880 & -1.7298980 & 0.4568930 \\ \mathrm{C} & -7.6999060 & -2.4878220 & 0.9052390 & \mathrm{H} & 7.8727650 & -0.7496550 & 2.1793460 \\ \mathrm{C} & -6.8065550 & -1.5653090 & 1.4439300 & \mathrm{H} & 6.8898050 & 0.3656490 & 4.1733730 \\ \mathrm{C} & -5.4524260 & -1.6398140 & 1.1363250 & \mathrm{H} & 4.4199270 & 0.4516400 & 4.4364280 \\ \mathrm{O} & -1.1308900 & 0.1823800 & -1.2405250 & \mathrm{H} & 2.9511450 & -0.5595230 & 2.7257450 \\ \mathrm{H} & 0.9281690 & -5.8070640 & -3.3340760 & \mathrm{H} & 4.9417020 & -3.6079610 & 0.4328610 \\ \mathrm{H} & -1.6347450 & 0.9391240 & 1.6597860 & \mathrm{H} & 4.5218190 & -5.6036680 & -0.9801360 \\ \mathrm{H} & -5.5045970 & -4.3384420 & -0.9150030 & \mathrm{H} & 3.0521330 & -6.8361770 & -2.5386610 \\ \mathrm{H} & -7.9507820 & -4.2017640 & -0.3548820 & \mathrm{H} & 0.2939410 & -3.5260630 & -2.5722620 \\ \mathrm{H} & -7.1843580 & -0.7857970 & 2.0991090 & \mathrm{H} & -2.4458220 & 3.1880600 & 2.3249690 \\ \mathrm{H} & -4.7517390 & -0.9199730 & 1.5429140 & \mathrm{O} & -3.4361750 & 6.4470440 & -1.3986830 \\ \mathrm{H} & -4.2565340 & 3.8517370 & -1.0583930 & \mathrm{O} & -9.0457550 & -2.4006770 & 1.2003910 \\ \mathrm{H} & -0.9367990 & 6.4870200 & -1.8235370 & \mathrm{C} & -4.8195800 & 6.3002310 & -1.1671590 \\ \mathrm{H} & 0.5637970 & 4.4960310 & -1.9252000 & \mathrm{H} & -5.0152400 & 5.8317870 & -0.1950740 \\ \mathrm{H} & -0.3447270 & 2.2107750 & -1.6131940 & \mathrm{H} & -5.2386590 & 7.3068430 & -1.1748090 \\ \mathrm{H} & -0.9033460 & 5.1414910 & 2.2440530 & \mathrm{H} & -5.2948460 & 5.7012260 & -1.9531680 \\ \mathrm{H} & 1.4345330 & 4.8490990 & 1.5037780 & \mathrm{C} & -9.3917090 & -3.0927280 & 2.3876130 \\ \mathrm{H} & 3.2177260 & 3.3862680 & 0.5776330 & \mathrm{H} & -10.4657550 & -2.9622590 & 2.5316430 \\ \mathrm{H} & 5.2424080 & 2.3560370 & 1.2838360 & \mathrm{H} & -9.1607130 & -4.1619380 & 2.3009450 \\ \mathrm{H} & 7.5400960 & 2.2826980 & 0.3705970 & \mathrm{H} & -8.8544040 & -2.6853940 & 3.2532100\end{array}$

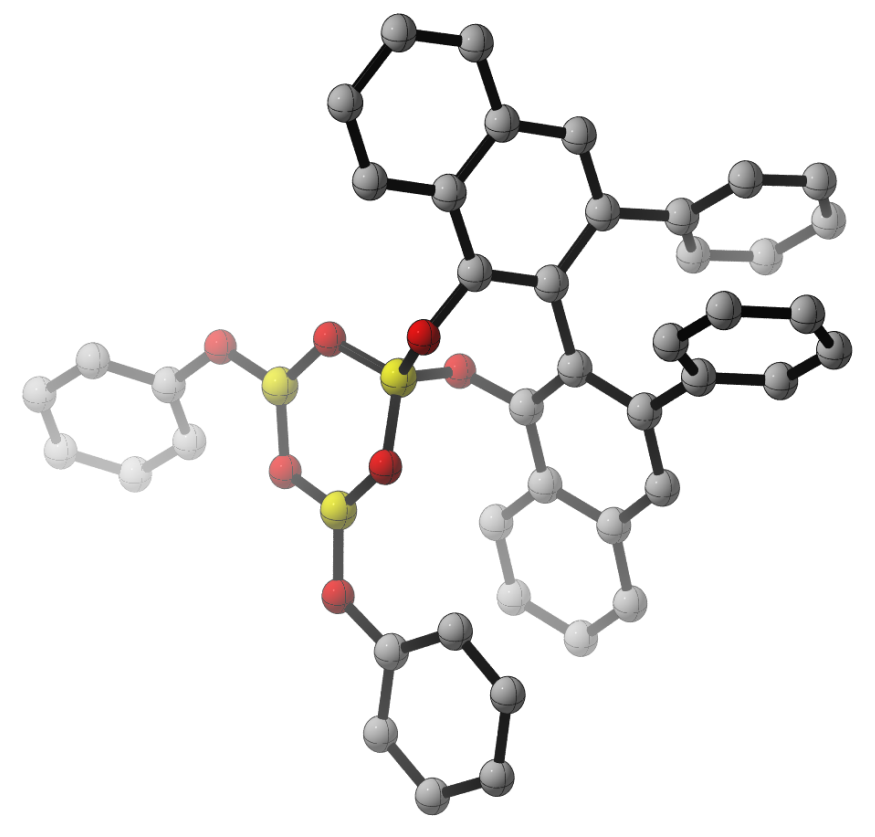

Entry 3 
File Name: cat3

RRHO Thermochemistry $(T=298)$

Zero-point correction $(\mathrm{RRHO})=$

Thermal correction to Energy $(\mathrm{RRHO})=$

0.652350 (Hartree/Particle)

0.692509

Thermal correction to Enthalpy $(\mathrm{RRHO})=$

0.693452

Thermal correction to Gibbs Free Energy $(\mathrm{RRHO})=0.578328$

Sum of electronic and zero-point Energies (RRHO)= $\quad-2295.001058$

Sum of electronic and thermal Energies $(\mathrm{RRHO})=$

$-2294.960899$

Sum of electronic and thermal Enthalpies $(\mathrm{RRHO})=$

$-2294.959956$

Sum of electronic and thermal Free Energies $(\mathrm{RRHO})=\quad-2295.075080$

Quasi-RRHO Thermochemistry ( $T=298$, v0 $=100 \mathrm{~cm}-1)$

$\begin{array}{lc}\text { Zero-point correction }(\mathrm{QRRHO})= & 0.652350 \text { (Hartree/Particle) } \\ \text { Thermal correction to Energy }(\mathrm{QRRHO})= & 0.692509 \\ \text { Thermal correction to Enthalpy (QRRHO)= } & 0.693452 \\ \text { Thermal correction to Gibbs Free Energy (QRRHO)= } & 0.587252 \\ \text { Sum of electronic and zero-point Energies (QRRHO)= } & -2295.001058 \\ \text { Sum of electronic and thermal Energies (QRRHO)= } & -2294.960899 \\ \text { Sum of electronic and thermal Enthalpies (QRRHO)= } & -2294.959956 \\ \text { Sum of electronic and thermal Free Energies (QRRHO)= } & -2295.066156\end{array}$

Quasi-harmonic Thermochemistry $(T=298$, cutoff $=100 \mathrm{~cm}-1)$

Zero-point correction $(\mathrm{QHO})=$

Thermal correction to Energy $(\mathrm{QHO})=$

0.652350 (Hartree/Particle)

Thermal correction to Enthalpy $(\mathrm{QHO})=$

0.692679

0.693452

Thermal correction to Gibbs Free Energy $(\mathrm{QHO})=0.588896$

Sum of electronic and zero-point Energies $(\mathrm{QHO})=\quad-2295.001058$

Sum of electronic and thermal Energies $(\mathrm{QHO})=\quad-2294.960729$

Sum of electronic and thermal Enthalpies $(\mathrm{QHO})=\quad-2294.959956$

Sum of electronic and thermal Free Energies $(\mathrm{QHO})=\quad-2295.064512$

$\begin{array}{lrrr}\text { C } & 3.9963290 & 0.3586370 & -1.7026090 \\ \text { C } & 3.8120770 & 1.1937600 & -0.5936640 \\ \text { C } & 4.9207860 & 1.8749630 & -0.0777470 \\ \text { C } & 6.1812990 & 1.7215890 & -0.6456960 \\ \text { C } & 6.3557820 & 0.8801050 & -1.7417130 \\ \text { C } & 5.2575440 & 0.2020990 & -2.2689880 \\ \text { C } & 2.4696260 & 1.3930720 & 0.0162200 \\ \text { C } & 1.5622950 & 0.3051650 & 0.2527030 \\ \text { C } & 0.2909770 & 0.5883710 & 0.7315310 \\ \text { C } & -0.1123250 & 1.9224650 & 1.0448350 \\ \text { C } & 0.8045660 & 2.9830360 & 0.8355810 \\ \text { C } & 2.0867470 & 2.6829860 & 0.3128190 \\ \text { C } & 0.3830830 & 4.3124010 & 1.1018720 \\ \text { C } & -0.8886540 & 4.5685510 & 1.5497030\end{array}$

$\begin{array}{lr}\text { C } & -1.7976560 \\ \text { C } & -1.4157540 \\ \text { C } & 1.8982280 \\ \text { C } & 1.0160760 \\ \text { C } & 1.3108500 \\ \text { C } & 2.5204200 \\ \text { C } & 3.3843330 \\ \text { C } & 3.0894680 \\ \text { C } & 0.4138510 \\ \text { C } & 0.7166520 \\ \text { C } & 1.9274760 \\ \text { C } & 2.8054210 \\ \text { O } & -0.1056500 \\ \text { B } & -1.1721420\end{array}$

3.5046830

1.7650370

2.2082060

1.5225260

$-1.1282000$

0.0160400

$-1.8952430$

$-0.7386660$

$-3.2593540$

$-1.0583290$

$-3.8353950$

$-0.5958330$

$-3.0533460$

0.2081940

$-1.7464630$

0.5270070

$-4.0396090$

$-1.8298940$

$-5.3428720$

$-2.1389840$

$-5.9213610$

$-1.6858530$

$-5.1859290$

$-0.9297150$

$-1.3798080$

$-1.2647510$

$-0.8697010$

$-0.3984240$ 


\begin{tabular}{|c|c|c|c|c|c|c|c|}
\hline 0 & -0.6115150 & -0.3993680 & 0.8911390 & $\mathrm{H}$ & -6.2142570 & -4.0529420 & $-0.241288 c$ \\
\hline $\mathrm{C}$ & 4.0283460 & -1.0334110 & 1.4355370 & $\mathrm{H}$ & -8.6348780 & -3.8021000 & 0.303096 \\
\hline $\mathrm{C}$ & 3.5680690 & -0.2775130 & 2.5205880 & $\mathrm{H}$ & -9.4010640 & -1.8762530 & 1.678140 \\
\hline $\mathrm{C}$ & 4.4659900 & 0.3614910 & 3.3700150 & $\mathrm{H}$ & -7.7384670 & -0.2235470 & $2.50106 \varepsilon$ \\
\hline $\mathrm{C}$ & 5.8389280 & 0.2537050 & 3.1539770 & $\mathrm{H}$ & -5.3347930 & -0.4864030 & 1.94580 \\
\hline C & 6.3073820 & -0.5043130 & 2.0836410 & $\mathrm{H}$ & -4.8086750 & 4.0475380 & -1.24450 \\
\hline C & 5.4092370 & -1.1403780 & 1.2334590 & $\mathrm{H}$ & -3.8476340 & 6.2458530 & -1.91649 \\
\hline $\mathrm{O}$ & -2.1575980 & -1.8997760 & -0.0882440 & $\mathrm{H}$ & -1.4275420 & 6.4153030 & -2.48264 \\
\hline $\mathrm{B}$ & -3.4353110 & -1.5399940 & 0.0972110 & $\mathrm{H}$ & -0.0082830 & 4.3745860 & -2.37653 \\
\hline $\mathrm{O}$ & -3.9016850 & -0.2590060 & -0.1483370 & $\mathrm{H}$ & -0.9782210 & 2.1987930 & -1.73367 \\
\hline$B$ & -3.0363650 & 0.6237820 & -0.7812420 & $\mathrm{H}$ & -1.2063400 & 5.5909870 & 1.732160 \\
\hline $\mathrm{O}$ & -3.5953750 & 1.8609490 & -1.0477250 & $\mathrm{H}$ & 1.0840290 & 5.1255720 & 0.927819 \\
\hline $\mathrm{C}$ & -2.9586000 & 2.9989220 & -1.4519480 & $\mathrm{H}$ & 2.7650890 & 3.5042910 & 0.095240 \\
\hline $\mathrm{C}$ & -1.6020450 & 3.0818090 & -1.7805990 & $\mathrm{H}$ & 4.7937990 & 2.4945390 & 0.806062 \\
\hline $\mathrm{C}$ & -1.0679820 & 4.3161550 & -2.1427780 & $\mathrm{H}$ & 7.0317800 & 2.2453520 & $-0.21844 \mathrm{C}$ \\
\hline $\mathrm{C}$ & -1.8593950 & 5.4607410 & -2.1980150 & $\mathrm{H}$ & 7.3399940 & 0.7520790 & -2.182932 \\
\hline C & -3.2127130 & 5.3650030 & -1.8798570 & $\mathrm{H}$ & 5.3813120 & -0.4497310 & -3.12892 \\
\hline $\mathrm{C}$ & -3.7596170 & 4.1426670 & -1.5069220 & $\mathrm{H}$ & 3.1429340 & -0.1672310 & -2.120156 \\
\hline $\mathrm{O}$ & -4.3281400 & -2.5106830 & 0.5312450 & $\mathrm{H}$ & 5.7739020 & -1.6914720 & 0.370872 \\
\hline $\mathrm{C}$ & -5.6420840 & -2.2858340 & 0.8153460 & $\mathrm{H}$ & 7.3746290 & -0.5854730 & 1.897407 \\
\hline $\mathrm{C}$ & -6.5708450 & -3.2199390 & 0.3559180 & $\mathrm{H}$ & 6.5373730 & 0.7576040 & 3.815829 \\
\hline $\mathrm{C}$ & -7.9187090 & -3.0709190 & 0.6666890 & $\mathrm{H}$ & 4.0903770 & 0.9434640 & 4.206628 \\
\hline $\mathrm{C}$ & -8.3491430 & -1.9930100 & 1.4372710 & $\mathrm{H}$ & 2.4997380 & -0.1928090 & $2.69553 \mathrm{~S}$ \\
\hline C & -7.4152210 & -1.0666950 & 1.8975670 & $\mathrm{H}$ & 4.2766480 & -3.5170070 & 0.621122 \\
\hline $\mathrm{C}$ & -6.0643480 & -1.2074880 & 1.5953930 & $\mathrm{H}$ & 3.7326340 & -5.6263840 & -0.570915 \\
\hline $\mathrm{O}$ & -1.7863900 & 0.2670320 & -1.1034930 & $\mathrm{H}$ & 2.1562090 & -6.9537490 & -1.934662 \\
\hline $\mathrm{H}$ & 0.0247670 & -5.9382440 & -2.7271560 & $\mathrm{H}$ & -0.5127840 & -3.5776010 & $-2.15275 c$ \\
\hline $\mathrm{H}$ & -2.0993470 & 1.3802300 & 1.6850210 & $\mathrm{H}$ & -2.8035050 & 3.7197330 & 2.112939 \\
\hline
\end{tabular}




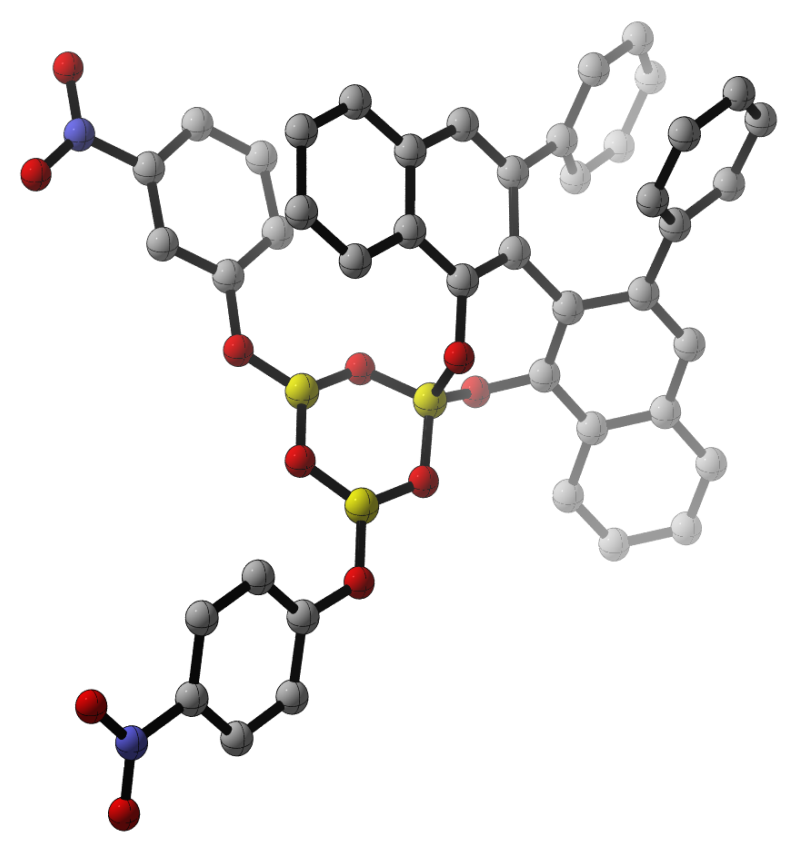

Entry 4

File Name: cat4

RRHO Thermochemistry $(T=298)$

Zero-point correction $(\mathrm{RRHO})=$

0.657709 (Hartree/Particle)

Thermal correction to Energy $(\mathrm{RRHO})=$

0.703162

Thermal correction to Enthalpy $(\mathrm{RRHO})=$

0.704106

Thermal correction to Gibbs Free Energy $(\mathrm{RRHO})=$

Sum of electronic and zero-point Energies $(\mathrm{RRHO})=$

0.575303

Sum of electronic and thermal Energies $(\mathrm{RRHO})=$

$-2703.865917$

Sum of electronic and thermal Enthalpies $(\mathrm{RRHO})=$

$-2703.820464$

Sum of electronic and thermal Free Energies $(\mathrm{RRHO})=$

$-2703.819520$

$-2703.948323$

Quasi-RRHO Thermochemistry $(T=298, v 0=100 \mathrm{~cm}-1)$

Zero-point correction (QRRHO)=

Thermal correction to Energy $(\mathrm{QRRHO})=$

0.657709 (Hartree/Particle)

Thermal correction to Enthalpy $(\mathrm{QRRHO})=$

0.703162

0.704106

Thermal correction to Gibbs Free Energy (QRRHO)=

Sum of electronic and zero-point Energies $(Q R R H O)=$

Sum of electronic and thermal Energies (QRRHO)=

0.586792

Sum of electronic and thermal Enthalpies (QRRHO)=

$-2703.865917$

$-2703.820464$

$-2703.819520$

Sum of electronic and thermal Free Energies $(\mathrm{QRRHO})=$

$-2703.936834$

Quasi-harmonic Thermochemistry $(T=298$, cutoff $=100 \mathrm{~cm}-1)$

Zero-point correction $(\mathrm{QHO})=$

Thermal correction to Energy $(\mathrm{QHO})=$

Thermal correction to Enthalpy $(\mathrm{QHO})=$
0.657709 (Hartree/Particle)

0.703377

0.704106 
Thermal correction to Gibbs Free Energy (QHO)= Sum of electronic and zero-point Energies $(\mathrm{QHO})=$ Sum of electronic and thermal Energies $(\mathrm{QHO})=$ Sum of electronic and thermal Enthalpies $(\mathrm{QHO})=$ Sum of electronic and thermal Free Energies (QHO)=

$\begin{array}{lrrr}\text { C } & 4.7525880 & 0.3110220 & -1.5033490 \\ \text { C } & 4.4993260 & 1.0320530 & -0.3298820 \\ \text { C } & 5.5639880 & 1.6940860 & 0.2920510 \\ \text { C } & 6.8494230 & 1.6310440 & -0.2352750 \\ \text { C } & 7.0933930 & 0.9009630 & -1.3959110 \\ \text { C } & 6.0392860 & 0.2436520 & -2.0286650 \\ \text { C } & 3.1298890 & 1.1308020 & 0.2428040 \\ \text { C } & 2.2531000 & -0.0036740 & 0.3336620 \\ \text { C } & 0.9552580 & 0.1935140 & 0.7802620 \\ \text { C } & 0.4954810 & 1.4734200 & 1.2143690 \\ \text { C } & 1.3819490 & 2.5775120 & 1.1518910 \\ \text { C } & 2.6913720 & 2.3721560 & 0.6505230 \\ \text { C } & 0.9007550 & 3.8592560 & 1.5285360 \\ \text { C } & -0.3975010 & 4.0289280 & 1.9411680 \\ \text { C } & -1.2734610 & 2.9186370 & 2.0180980 \\ \text { C } & -0.8341800 & 1.6658400 & 1.6670790 \\ \text { C } & 2.6507670 & -1.3983970 & -0.0146000 \\ \text { C } & 1.8334150 & -2.1254490 & -0.8733940 \\ \text { C } & 2.1865980 & -3.4494000 & -1.2872300 \\ \text { C } & 3.3915320 & -4.0251330 & -0.8126290 \\ \text { C } & 4.1910240 & -3.2861490 & 0.0924470 \\ \text { C } & 3.8389650 & -2.0196010 & 0.5015720 \\ \text { C } & 1.3540420 & -4.1908650 & -2.1625680 \\ \text { C } & 1.7153030 & -5.4550590 & -2.5589150 \\ \text { C } & 2.9221070 & -6.0321170 & -2.0937230 \\ \text { C } & 3.7376850 & -5.3348030 & -1.2383190 \\ \text { O } & 0.7209400 & -1.6018320 & -1.4165410 \\ \text { B } & -0.3906890 & -1.1612320 & -0.5803800 \\ \text { O } & 0.0721360 & -0.8266270 & 0.7834590 \\ \text { C } & 4.7126470 & -1.3557450 & 1.5076740 \\ \text { C } & 4.1804820 & -0.7090480 & 2.6297840 \\ \text { C } & 5.0182820 & -0.1142570 & 3.5682550 \\ \text { C } & 6.4021970 & -0.1598780 & 3.4060070 \\ \text { C } & 6.9421510 & -0.8101170 & 2.2990120 \\ \text { C } & 6.1038310 & -1.4006780 & 1.3592980 \\ \text { O } & -1.4174460 & -2.1953730 & -0.4451250 \\ \text { B } & -2.6913420 & -1.8218620 & -0.3014260 \\ \text { O } & -3.1257590 & -0.5146650 & -0.4336460 \\ \text { B } & -2.2039700 & 0.4052960 & -0.9161910 \\ \text { O } & -2.7158310 & 1.6865350 & -1.0682690 \\ \text { C } & -2.0129370 & 2.8313290 & -1.2697710 \\ \text { C } & -0.6495140 & 2.8922790 & -1.5850590 \\ \text { C } & -0.0346490 & 4.1317950 & -1.7474290 \\ & -0.7428080 & 5.3193850 & -1.6117370\end{array}$

0.589272

$-2703.865917$

$-2703.820249$

$-2703.819520$

$-2703.934354$

C -2.0941580

C $\quad-2.7452740$

5.2223670

$-1.3058310$

4.0109420

$-1.1318900$

$-2.8172700$

$-0.0349050$

$-2.6264700$

0.2483140

$-3.6032370$

$-0.2078500$

$-3.5086660$

0.0874410

$-2.4295330$

0.8459860

$-1.4524560$

1.3152690

$-1.5519640$

1.0189290

0.0518250

$-1.2128610$

$-6.0210560$

$-3.2261970$

1.7234800

$-1.4907010 \quad 0.8024930$

$-0.7930750$

$-4.4282420$

$-0.2579010$

$-7.8906880$

$-4.2513720$

1.9075750

$-7.1375460$

$-0.6311060$

1.3698540

$-4.7070380$

$-0.8016900$

3.9722570

$-0.8844530$

$-0.2779240$

6.2889000

$-1.7333570$

1.0263080

4.1629390

$-1.9744090$

$-0.0789740$

1.9773580

$-1.6795700$

2.2091660

1.5758870

5.0174710

1.4641060

3.3482060

4.7094330

3.2318530

5.3837430

7.6652110

2.2221150

0.5419330

1.2244720

0.2733620

8.0975640

6.2173830

3.9343110

6.5240800

2.1368600

0.8435990

$-0.3208200$

$-0.1982810$

$-1.8057420$

$-2.9392480$

$-2.0035120$

0.4713800

2.1547890

4.1376920

4.4324120

2.7631460

0.5078710

$-0.8703710$

$-2.4130310$

$-2.4955130$

2.3474800

$-1.1488760$

1.1633110

0.7462620

$-1.2906020$

1.8305860

$-0.8835890$ 
Figure SI-1 Catalyst-imine structures used to determine the $\mathrm{H}$-bond distances seen in Table 4.
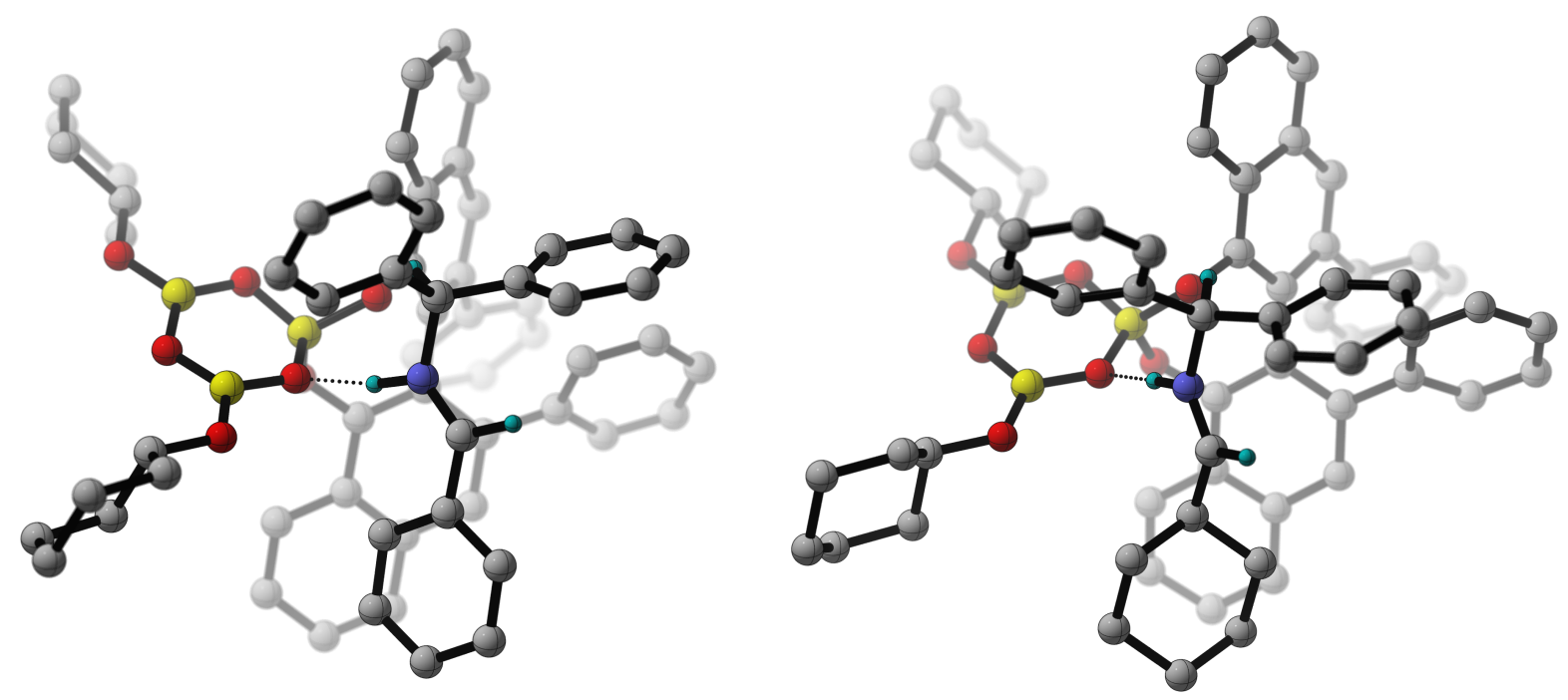

Boroxinate-H-Imine Complex 4a

Boroxinate-H-Imine Complex $4 \mathrm{e}$
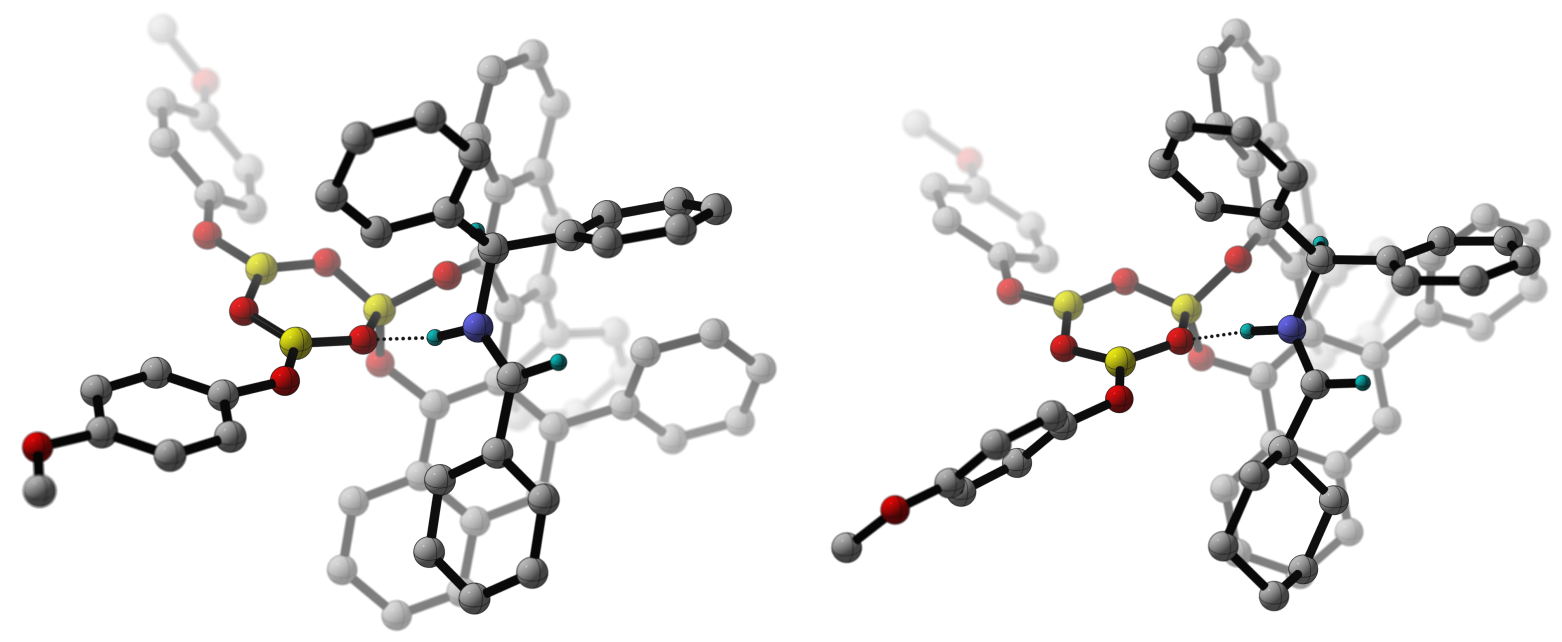

Boroxinate-H-Imine Complex 4b

Boroxinate-H-Imine Complex $4 \mathbf{f}$ 
Figure SI-1 (Cont'd)
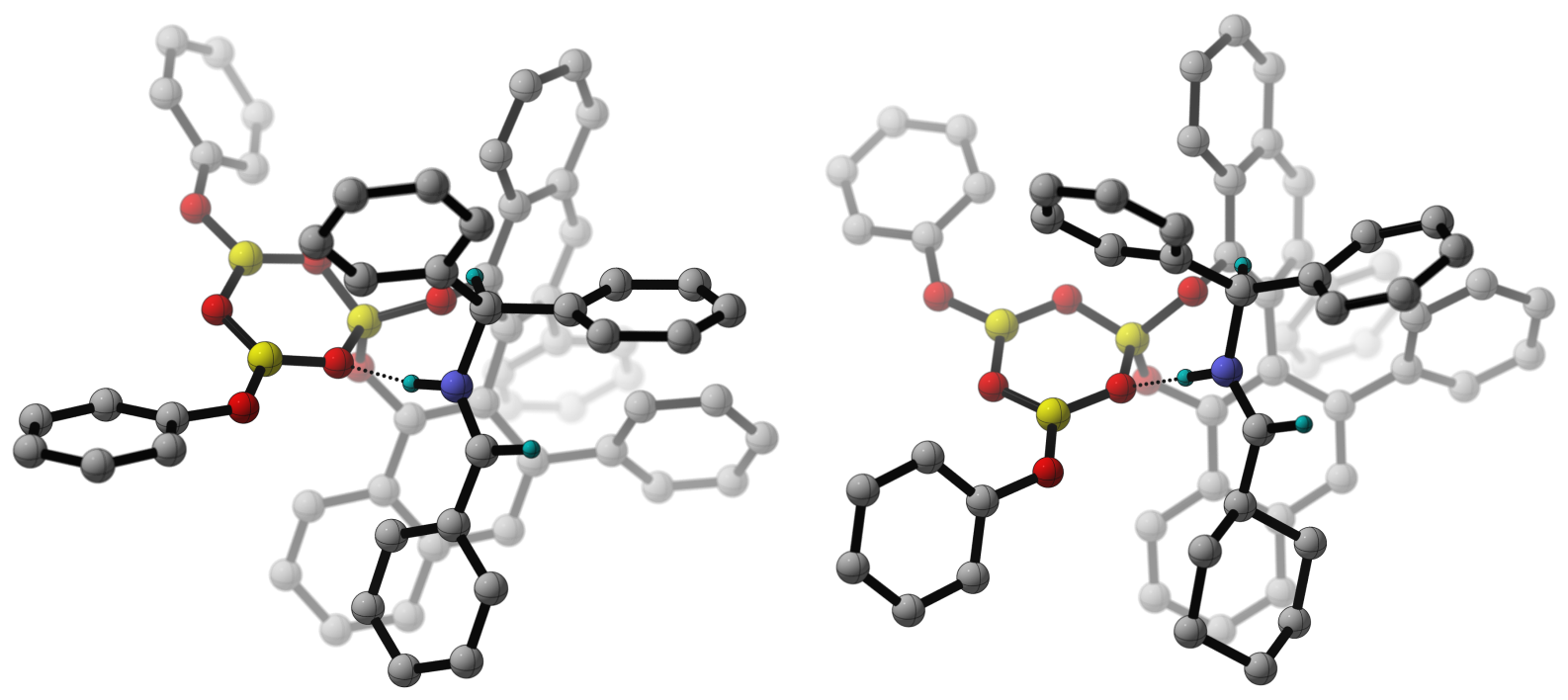

Boroxinate-H-Imine Complex 4c

Boroxinate-H-Imine Complex $\mathbf{4 g}$
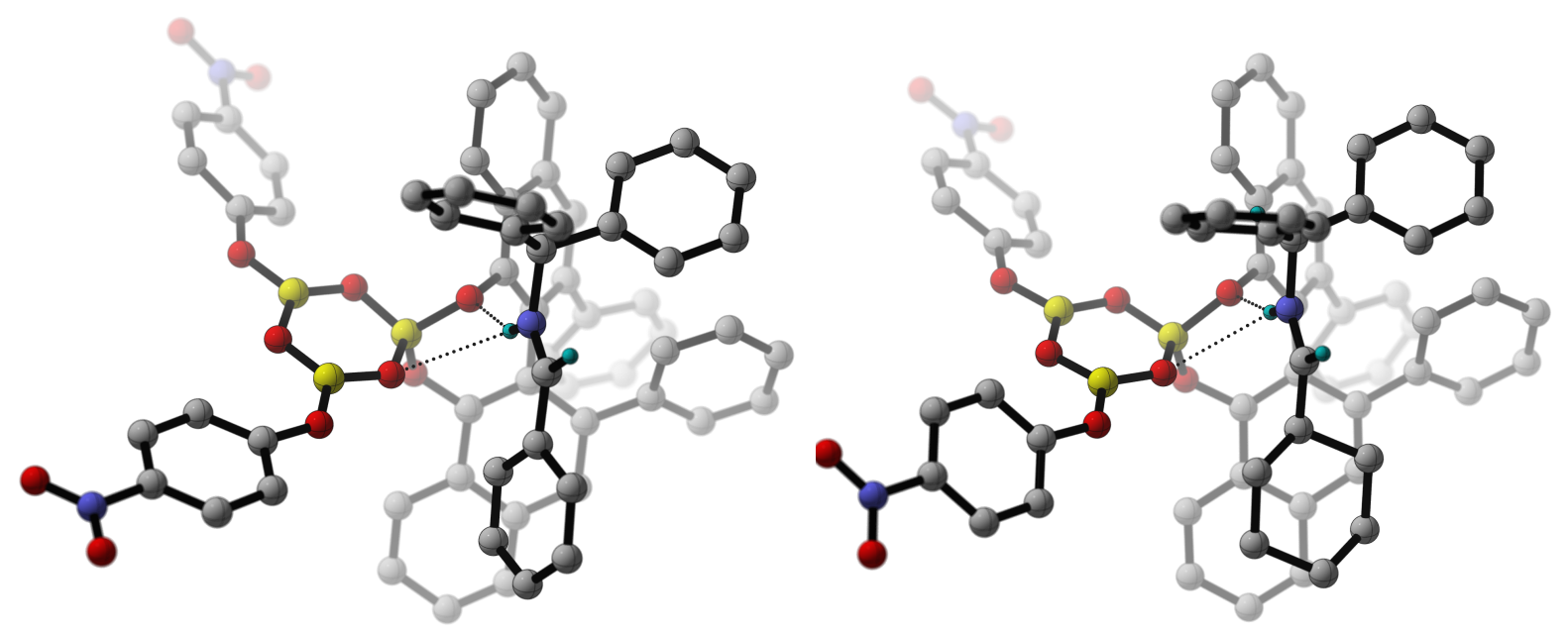

Boroxinate-H-Imine Complex 4d

Boroxinate-H-Imine Complex 4h 


\section{Coordinates Used for Hydrogen Bonding Interactions in the Catalyst-Iminium Complex:}

Optimizations of catalyst-borox-iminium complexes were carried out at the M06-2X/6-31G* level of theory as implemented by the Gaussian 16 software package with bulk solvent effects accounted for with the self-consistent reaction field polarizable continuum model (PCM) as implemented for toluene.

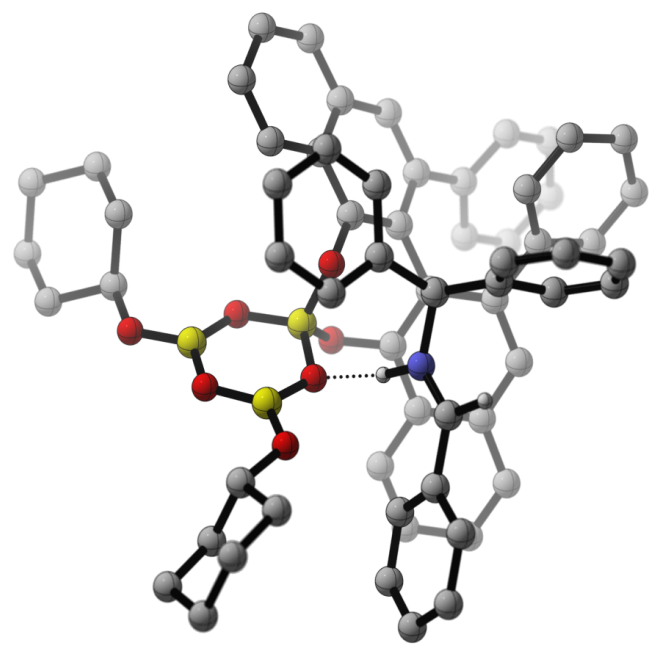

Boroxinate-H-Iminium Complex 4a

File Name: catim4a

RRHO Thermochemistry $(T=298)$

Zero-point correction $(\mathrm{RRHO})=$

Thermal correction to Energy $(\mathrm{RRHO})=$

Thermal correction to Enthalpy $(\mathrm{RRHO})=$

\subsection{8 (Hartree/Particle)}

1.187473

1.188417

Thermal correction to Gibbs Free Energy $(\mathrm{RRHO})=$

Sum of electronic and zero-point Energies $(\mathrm{RRHO})=$

1.026235

Sum of electronic and thermal Energies $(\mathrm{RRHO})=$

$-3129.018780$

Sum of electronic and thermal Enthalpies (RRHO)=

$-3128.957425$

Sum of electronic and thermal Free Energies (RRHO)= $\quad-3129.118663$

Quasi-RRHO Thermochemistry $(T=298, \mathrm{v} 0=100 \mathrm{~cm}-1)$

Zero-point correction $(\mathrm{QRRHO})=$

Thermal correction to Energy $(\mathrm{QRRHO})=$

Thermal correction to Enthalpy $(Q R R H O)=$

Thermal correction to Gibbs Free Energy (QRRHO)=

Sum of electronic and zero-point Energies $(\mathrm{QRRHO})=$

Sum of electronic and thermal Energies $(\mathrm{QRRHO})=$

Sum of electronic and thermal Enthalpies (QRRHO)=

Sum of electronic and thermal Free Energies $(Q R R H O)=$
1.126118 (Hartree/Particle)

1.187473

1.188417

1.042350

$-3129.018780$

$-3128.957425$

$-3128.956481$

$-3129.102548$ 
Quasi-harmonic Thermochemistry $(T=298$, cutoff $=100 \mathrm{~cm}-1)$

Zero-point correction $(\mathrm{QHO})=$

Thermal correction to Energy $(\mathrm{QHO})=$

Thermal correction to Enthalpy $(\mathrm{QHO})=$

Thermal correction to Gibbs Free Energy (QHO)=

Sum of electronic and zero-point Energies (QHO)=

Sum of electronic and thermal Energies $(\mathrm{QHO})=$

Sum of electronic and thermal Enthalpies $(\mathrm{QHO})=$

Sum of electronic and thermal Free Energies (QHO)=
1.126118 (Hartree/Particle)

1.187795

1.188417

1.045486

$-3129.018780$

$-3128.957103$

$-3128.956481$

$-3129.099412$

$\begin{array}{lrrr}\text { C } & 4.0197040 & -1.1760430 & 1.0080360 \\ \text { C } & 3.9513690 & -1.6441280 & -0.3108010 \\ \text { C } & 5.0487520 & -2.3444640 & -0.8256590 \\ \text { C } & 6.1809570 & -2.5703500 & -0.0496200 \\ \text { C } & 6.2356680 & -2.1015340 & 1.2604060 \\ \text { C } & 5.1491640 & -1.4052030 & 1.7866280 \\ \text { C } & 2.7406680 & -1.4573590 & -1.1594990 \\ \text { C } & 2.0246670 & -0.2135690 & -1.2260590 \\ \text { C } & 0.8783850 & -0.1406100 & -2.0084090 \\ \text { C } & 0.4408190 & -1.2464700 & -2.7994460 \\ \text { C } & 1.1517490 & -2.4704580 & -2.7207520 \\ \text { C } & 2.2888990 & -2.5458930 & -1.8780940 \\ \text { C } & 0.6911760 & -3.5752260 & -3.4864850 \\ \text { C } & -0.3953120 & -3.4488050 & -4.3154080 \\ \text { C } & -1.0787320 & -2.2113750 & -4.4175980 \\ \text { C } & -0.6776360 & -1.1346880 & -3.6655690 \\ \text { H } & -1.9240050 & -2.1198870 & -5.0936510 \\ \text { C } & 2.4274360 & 1.0134710 & -0.4821410 \\ \text { C } & 1.4565960 & 1.6297470 & 0.2961480 \\ \text { C } & 1.7665910 & 2.7562110 & 1.1174410 \\ \text { C } & 3.0886100 & 3.2685900 & 1.1089890 \\ \text { C } & 4.0529670 & 2.6620780 & 0.2656840 \\ \text { C } & 3.7488290 & 1.5738330 & -0.5212980 \\ \text { C } & 0.7811850 & 3.3622680 & 1.9351140 \\ \text { C } & 1.1007670 & 4.4466310 & 2.7154370 \\ \text { C } & 2.4184300 & 4.9665520 & 2.7089520 \\ \text { C } & 3.3879510 & 4.3914940 & 1.9253100 \\ \text { H } & -0.2191370 & 2.9406700 & 1.9290500 \\ \text { O } & 0.2029370 & 1.1413460 & 0.3539170 \\ \text { B } & -0.6560550 & 1.2171590 & -0.8472580 \\ \text { O } & 0.1439580 & 0.9884680 & -2.0531510 \\ \text { C } & 4.8105900 & 1.0295730 & -1.4101210 \\ \text { C } & 6.1096620 & 0.8558730 & -0.9183730 \\ \text { C } & 7.1181950 & 0.3506960 & -1.7318530 \\ \text { C } & 6.8441480 & 0.0095640 & -3.0542300 \\ \text { C } & 5.5569350 & 0.1887170 & -3.5581110 \\ \text { C } & 4.5487800 & 0.6972930 & -2.7447570 \\ \text { O } & -1.6265130 & 0.1048860 & -0.6664680 \\ & & & \end{array}$

$\begin{array}{rrrr}\text { B } & -2.9171400 & 0.3451040 & -0.3057560 \\ \text { O } & -3.4554530 & 1.6062740 & -0.3073170 \\ \text { B } & -2.6289780 & 2.6725890 & -0.6705980 \\ \text { O } & -3.2337330 & 3.8943360 & -0.7231350 \\ \text { O } & -3.6459560 & -0.7537730 & 0.0664490 \\ \text { O } & -1.3236740 & 2.4914180 & -0.9633130 \\ \text { N } & -0.7293460 & -2.0423670 & 0.7753840 \\ \mathrm{C} & -1.2798160 & -3.2042220 & 0.7687090 \\ \mathrm{C} & -2.2555000 & -3.6838230 & -0.1891120 \\ \mathrm{C} & -2.9315050 & -4.8708500 & 0.1317590 \\ \mathrm{C} & -3.9061660 & -5.3675140 & -0.7226820 \\ \mathrm{C} & -4.1860660 & -4.6929080 & -1.9113920 \\ \mathrm{C} & -3.4921100 & -3.5291100 & -2.2478750 \\ \mathrm{C} & -2.5318440 & -3.0147370 & -1.3890310 \\ \mathrm{C} & 0.2553600 & -1.5801600 & 1.7831580 \\ \mathrm{C} & 0.8835280 & -2.7483000 & 2.5234760 \\ \mathrm{C} & 0.5133360 & -3.0812390 & 3.8274170 \\ \mathrm{C} & 1.0995450 & -4.1742070 & 4.4642650 \\ \mathrm{C} & 2.0573110 & -4.9385480 & 3.8036840 \\ \mathrm{C} & 2.4246060 & -4.6148650 & 2.4979960 \\ \mathrm{C} & 1.8359670 & -3.5287320 & 1.8602500 \\ \mathrm{C} & -0.3700050 & -0.5341060 & 2.6893960 \\ \mathrm{C} & 0.4895200 & 0.2537510 & 3.4587610 \\ \mathrm{C} & -0.0221910 & 1.2160740 & 4.3196110 \\ \mathrm{C} & -1.4010230 & 1.4108980 & 4.4109740 \\ \mathrm{C} & -2.2584150 & 0.6315840 & 3.6420390 \\ \mathrm{C} & -1.7462670 & -0.3474690 & 2.7886500 \\ \mathrm{H} & 2.6592670 & 5.8261080 & 3.3274850 \\ \mathrm{H} & 4.3995970 & 4.7892150 & 1.9148550 \\ \mathrm{H} & 5.0469600 & 3.0986120 & 0.2117610 \\ \mathrm{H} & 6.3134150 & 1.0779820 & 0.1255360 \\ \mathrm{H} & 8.1152910 & 0.2061430 & -1.3260660 \\ \mathrm{H} & 7.6282700 & -0.3914040 & -3.6895770 \\ \mathrm{H} & 5.3367420 & -0.0622350 & -4.5915040 \\ \mathrm{H} & 3.5499050 & 0.8434730 & -3.1449370 \\ \mathrm{H} & 5.0256660 & -2.6735680 & -1.8606510 \\ \mathrm{H} & 7.0283660 & -3.0987690 & -0.4763270 \\ \mathrm{H} & 7.1196510 & -2.2732480 & 1.8670760\end{array}$




\begin{tabular}{|c|c|c|c|c|c|c|c|}
\hline $\mathrm{H}$ & 5.1788550 & -1.0388220 & 2.8083650 & $\mathrm{H}$ & -4.1313390 & 6.3207000 & -0.7128950 \\
\hline $\mathrm{H}$ & 3.1854090 & -0.6200630 & 1.4269190 & $\mathrm{H}$ & -3.9921570 & 5.7788700 & -2.3879580 \\
\hline $\mathrm{H}$ & 2.8137190 & -3.4948890 & -1.7957170 & C & -0.8620420 & 6.7876730 & -0.2069300 \\
\hline $\mathrm{H}$ & 1.2251920 & -4.5193650 & -3.4143510 & $\mathrm{H}$ & -2.3037220 & 5.6729180 & 0.9674500 \\
\hline $\mathrm{H}$ & -0.7317500 & -4.2975620 & -4.9032740 & $\mathrm{H}$ & -0.9302540 & 4.7039070 & 0.4015060 \\
\hline $\mathrm{H}$ & 1.0224270 & -1.0807770 & 1.1777230 & C & -1.8173680 & 7.8913280 & -0.668300 \\
\hline $\mathrm{H}$ & -1.0266630 & -1.2931590 & 0.0986020 & $\mathrm{H}$ & -3.3611140 & 8.2025360 & -2.166435 \\
\hline $\mathrm{H}$ & -0.9806000 & -3.8839310 & 1.5653430 & $\mathrm{H}$ & -1.9939680 & 7.2380650 & $-2.71567 c$ \\
\hline $\mathrm{H}$ & -2.6967000 & -5.3910610 & 1.0564880 & $\mathrm{H}$ & -0.2845310 & 7.1121350 & $0.66561 \mathrm{c}$ \\
\hline $\mathrm{H}$ & -4.4391990 & -6.2777320 & -0.4703130 & $\mathrm{H}$ & -0.1356370 & 6.5813640 & -1.004810 \\
\hline $\mathrm{H}$ & -4.9415410 & -5.0834720 & -2.5862850 & $\mathrm{H}$ & -1.2591530 & 8.7967760 & -0.931152 \\
\hline $\mathrm{H}$ & -3.6977890 & -3.0194100 & -3.1835080 & $\mathrm{H}$ & -2.4878900 & 8.1576190 & 0.160830 \\
\hline $\mathrm{H}$ & -1.9976920 & -2.1062250 & -1.6511020 & C & -5.0425150 & -0.6849170 & 0.32760 \\
\hline $\mathrm{H}$ & -0.2228110 & -2.4773620 & 4.3495400 & C & -5.8221520 & -0.5353620 & $-0.97608 \varepsilon$ \\
\hline $\mathrm{H}$ & 2.1152810 & -3.2759470 & 0.8402330 & C & -5.4285390 & -1.9715130 & 1.047604 \\
\hline $\mathrm{H}$ & -2.4296970 & -0.9395910 & 2.1868530 & $\mathrm{H}$ & -5.2498430 & 0.1816930 & 0.97294 \\
\hline $\mathrm{H}$ & 1.5655190 & 0.1162790 & 3.3741180 & C & -7.3313260 & -0.5934040 & -0.726317 \\
\hline $\mathrm{H}$ & 0.3414460 & 4.9102880 & 3.3388500 & $\mathrm{H}$ & -5.5205690 & -1.3597130 & -1.637906 \\
\hline $\mathrm{H}$ & -1.1909790 & -0.1805830 & -3.7166450 & $\mathrm{H}$ & -5.5396600 & 0.4045060 & -1.461831 \\
\hline $\mathrm{H}$ & 0.6563010 & 1.8321460 & 4.9012300 & C & -6.9358900 & -2.0334450 & 1.304127 \\
\hline $\mathrm{H}$ & -1.8010780 & 2.1723200 & 5.0731500 & $\mathrm{H}$ & -5.1211230 & -2.8141650 & $0.41072 \varepsilon$ \\
\hline $\mathrm{H}$ & -3.3325330 & 0.7804540 & 3.6997900 & $\mathrm{H}$ & -4.8637680 & -2.0502780 & 1.984813 \\
\hline $\mathrm{H}$ & 3.1757510 & -5.2012860 & 1.9783910 & C & -7.7182370 & -1.8861810 & -0.00361 \\
\hline $\mathrm{H}$ & 2.5196100 & -5.7829700 & 4.3053150 & $\mathrm{H}$ & -7.8714550 & -0.5056320 & -1.674748 \\
\hline $\mathrm{H}$ & 0.8103780 & -4.4220040 & 5.4806780 & $\mathrm{H}$ & -7.6302000 & 0.2669850 & -0.112067 \\
\hline C & -2.4591680 & 5.0399950 & -1.0694120 & $\mathrm{H}$ & -7.1935570 & -2.9734100 & 1.803527 \\
\hline C & -3.4165690 & 6.1334550 & -1.5259040 & $\mathrm{H}$ & -7.2226220 & -1.2224390 & $1.98780 \leqq$ \\
\hline C & -1.6239280 & 5.5015720 & 0.1203710 & $\mathrm{H}$ & -8.7956900 & -1.9070620 & 0.192424 \\
\hline $\mathrm{H}$ & -1.7801470 & 4.7793340 & -1.8935630 & $\mathrm{H}$ & -7.4929290 & -2.7432520 & -0.65412 \\
\hline C & -2.6577960 & 7.4210120 & -1.8592930 & & & & \\
\hline
\end{tabular}




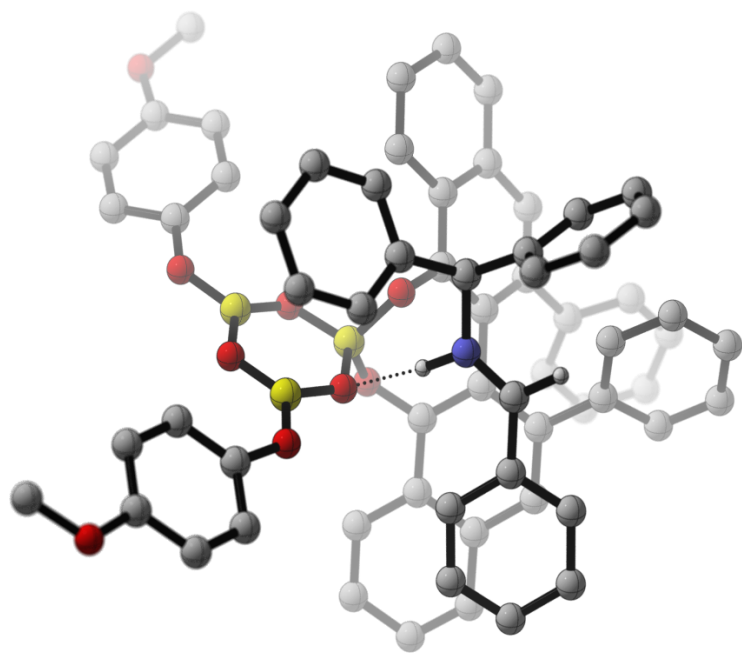

Boronxinate-H-Iminium Complex 4b

File Name: catim4b

RRHO Thermochemistry $(T=298)$

Zero-point correction $(\mathrm{RRHO})=$

Thermal correction to Energy $(\mathrm{RRHO})=$

1.050135 (Hartree/Particle)

Thermal correction to Enthalpy $(\mathrm{RRHO})=$

1.114383

1.115327

Thermal correction to Gibbs Free Energy $(\mathrm{RRHO})=$

Sum of electronic and zero-point Energies $(\mathrm{RRHO})=$

0.943116

Sum of electronic and thermal Energies $(\mathrm{RRHO})=$

$-3350.819070$

$-3350.754822$

Sum of electronic and thermal Enthalpies $(\mathrm{RRHO})=$

$-3350.753878$

Sum of electronic and thermal Free Energies $(\mathrm{RRHO})=\quad-3350.926089$

Quasi-RRHO Thermochemistry ( $T=298$, v0 = $100 \mathrm{~cm}-1)$

Zero-point correction (QRRHO)=

Thermal correction to Energy $(\mathrm{QRRHO})=$

Thermal correction to Enthalpy (QRRHO)=

Thermal correction to Gibbs Free Energy (QRRHO)=

Sum of electronic and zero-point Energies (QRRHO)=

Sum of electronic and thermal Energies (QRRHO)=

Sum of electronic and thermal Enthalpies (QRRHO)=

Sum of electronic and thermal Free Energies (QRRHO)=
1.050135 (Hartree/Particle)

1.114383

1.115327

0.962060

$-3350.819070$

$-3350.754822$

$-3350.753878$

$-3350.907145$

Quasi-harmonic Thermochemistry $(T=298$, cutoff $=100 \mathrm{~cm}-1)$

Zero-point correction $(\mathrm{QHO})=$ 1.050135 (Hartree/Particle)

Thermal correction to Energy $(\mathrm{QHO})=$

1.114733

Thermal correction to Enthalpy $(\mathrm{QHO})=$

1.115327

Thermal correction to Gibbs Free Energy (QHO)=

Sum of electronic and zero-point Energies $(\mathrm{QHO})=$

0.967060

$-3350.819070$ 
Sum of electronic and thermal Energies $(\mathrm{QHO})=$ Sum of electronic and thermal Enthalpies (QHO)= Sum of electronic and thermal Free Energies $(\mathrm{QHO})=$

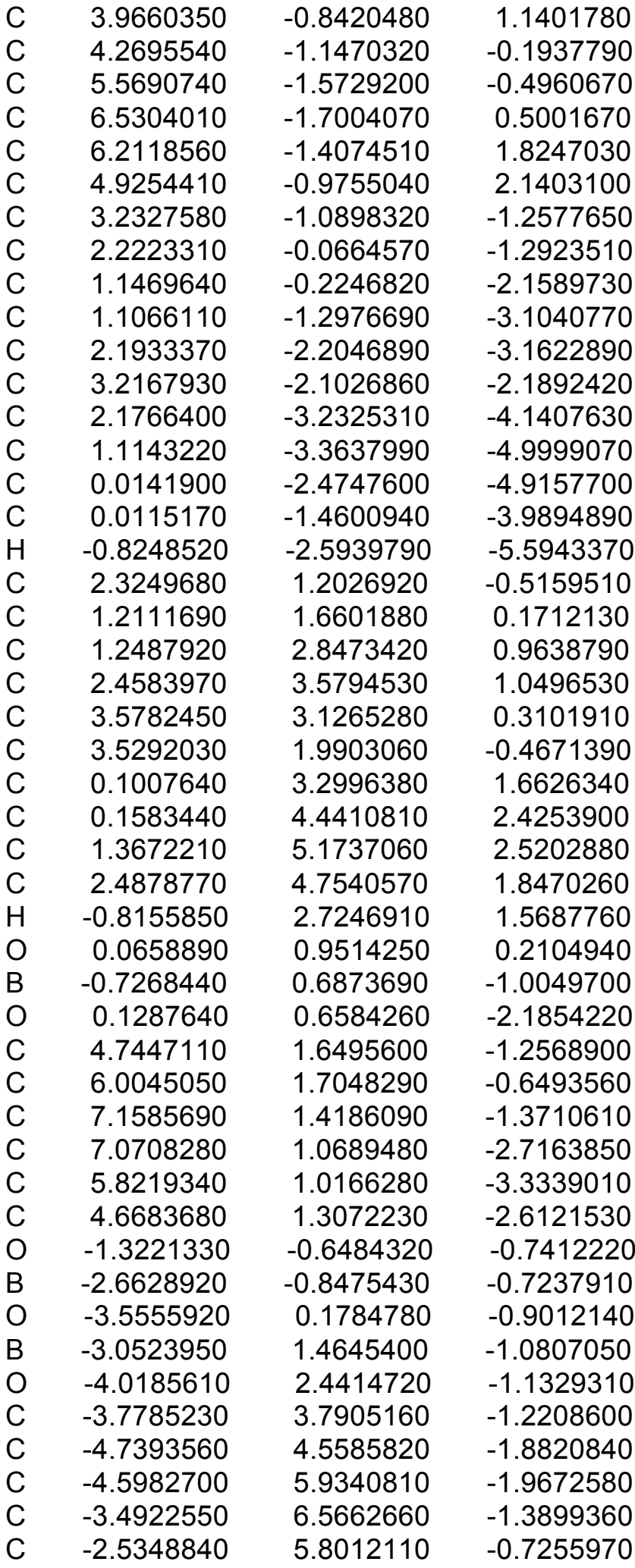

$-3350.754472$

$-3350.753878$ $-3350.902145$

C $\quad-2.6808420$

$-3.0660230$

$-4.3384260$

$-5.4831910$

$-6.7299630$

$-6.8357780$

$-5.6808290$

$-4.4464840$

$-1.7383870$

0.3379440

1.1367950

1.2397460

2.3284430

2.4577250

1.4917960

0.4030690

0.2720720

0.1436410

0.8617280

0.3755340

1.0635380

2.2348020

2.7166150

2.0328220

$-1.3462240$

$-1.7664530$

$-3.1210480$

$-4.0689920$

$-3.6544340$

$-2.2950020$

$-5.4104490$

$-7.6069010$

$-5.7772970$

$-3.5426390$

$-5.5942120$

$-5.3353330$

$-1.6650980$

$-1.9237320$

1.4026170

3.4149610

4.4857700

6.0694230

8.1253180

7.9690730

5.7443750

3.6986920

5.8354880
4.4157770

$-2.1524830$

$-2.6614460$

$-1.9082330$

$-2.5358470$

$-3.9149180$

$-4.6677370$

$-4.0455410$

1.7055380

$-1.4719290$

$-2.4794970$

$-3.4157480$

$-4.2995180$

$-5.2494320$

$-5.3274870$

$-4.4537600$

$-3.4907380$

$-0.5516910$

$-1.0205710$

$-2.0884730$

$-2.5087580$

$-1.8578820$

$-0.7869620$

$-0.3721350$

$-0.3311560$

0.8575070

1.1075560

0.1687760

$-1.0238290$

$-1.2759990$

$-0.8361850$

$-1.9271100$

$-5.7407070$

$-4.6179620$

4.0571070

6.5431600

6.2591990

3.8282070

6.0743700

5.3180070

3.7243690

1.9354770

1.4532520

0.8398020

0.7542840

1.2689090

$-1.7583450$
$-0.6401980$

$-0.5262230$

$-0.4727080$

$-0.2297940$

$-0.1602320$

$-0.3302340$

$-0.5690180$

$-0.6381450$

$-1.1917860$

1.4873150

1.4463350

0.3464560

0.3593880

$-0.6446890$

$-1.6472970$

$-1.6578750$

$-0.6696200$

2.6329560

3.8789870

4.6392510

5.7722730

6.1614010

5.4145170

4.2724280

2.8235900

3.4247150

3.6125280

3.2050080

2.6209890

2.4336540

$-0.0997910$

0.0260780

$-0.6980880$

$-0.8221580$

$-2.3240820$

$-2.4802380$

$-0.2685690$

$-0.1365790$

3.1256720

1.9105790

0.3208710

0.4107230

$-0.8773920$

$-3.2819230$

$-4.3847380$

$-3.1002720$

$-1.5327770$ 


$\begin{array}{rrrrrrrr}\mathrm{H} & 7.5362750 & -2.0153920 & 0.2386940 & \mathrm{H} & -0.7254250 & 4.7885940 & 2.9525500 \\ \mathrm{H} & 6.9618640 & -1.5060830 & 2.6034450 & \mathrm{H} & -0.8126410 & -0.7585780 & -3.9210030 \\ \mathrm{H} & 4.6647170 & -0.7443850 & 3.1692950 & \mathrm{H} & -3.4383550 & 2.0397250 & 4.0691460 \\ \mathrm{H} & 2.9721230 & -0.4785720 & 1.3899940 & \mathrm{H} & -5.1268600 & 0.3691220 & 3.3413500 \\ \mathrm{H} & 3.9711070 & -2.8839820 & -2.1481890 & \mathrm{H} & -4.3840050 & -1.7617780 & 2.3019390 \\ \mathrm{H} & 3.0157520 & -3.9229520 & -4.1850280 & \mathrm{H} & 3.6204160 & -0.2692250 & 5.7206940 \\ \mathrm{H} & 1.1102380 & -4.1534980 & -5.7454920 & \mathrm{H} & 2.7652070 & -2.1822400 & 7.0510080 \\ \mathrm{H} & 0.5808390 & 0.3992240 & 2.3157470 & \mathrm{H} & 0.6810490 & -3.3375960 & 6.3592210 \\ \mathrm{H} & -0.2192810 & -1.2113880 & 0.6487950 & \mathrm{O} & -3.4407610 & 7.9228000 & -1.5273800 \\ \mathrm{H} & 1.7617610 & -2.6446780 & 2.3226350 & \mathrm{O} & -8.0037590 & -4.6149540 & -0.2800150 \\ \mathrm{H} & 3.0732860 & -4.2239490 & 1.1475150 & \mathrm{C} & -2.3226800 & 8.5818080 & -0.9742730 \\ \mathrm{H} & 3.3036430 & -5.9284780 & -0.6443890 & \mathrm{H} & -2.4532910 & 9.6412320 & -1.1948900 \\ \mathrm{H} & 1.5894340 & -6.0704990 & -2.4331370 & \mathrm{H} & -2.2725550 & 8.4401620 & 0.1122810 \\ \mathrm{H} & -0.3306230 & -4.5047190 & -2.4559500 & \mathrm{H} & -1.3877130 & 8.2278670 & -1.4248830 \\ \mathrm{H} & -0.5772810 & -2.8133600 & -0.6878700 & \mathrm{C} & -9.1869240 & -3.8795990 & -0.0519800 \\ \mathrm{H} & -0.5491320 & -2.5802110 & 4.3462750 & \mathrm{H} & -10.0008480 & -4.6042620 & -0.0540410 \\ \mathrm{H} & 2.4022350 & 0.4687480 & 3.6878740 & \mathrm{H} & -9.1577210 & -3.3678300 & 0.9175350 \\ \mathrm{H} & -1.9903550 & -2.2060280 & 1.9601470 & \mathrm{H} & -9.3551700 & -3.1400700 & -0.8439050 \\ \mathrm{H} & -1.0256570 & 1.5919630 & 3.7325910 & & & & \end{array}$

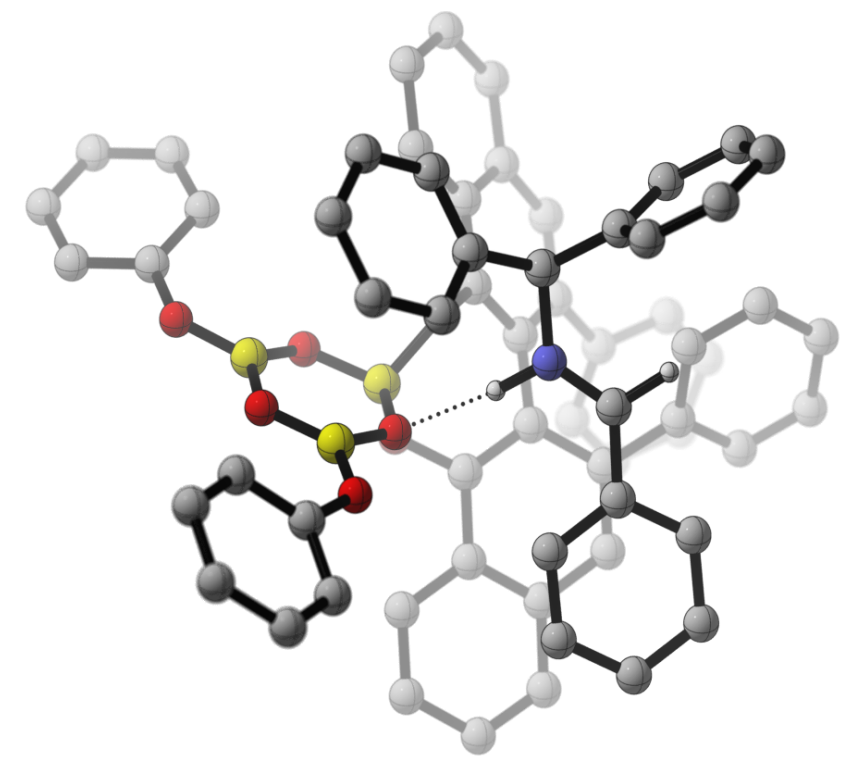

Boroxinate-H-Iminium Complex 4c

File Name: catim4c

RRHO Thermochemistry $(T=298)$

Zero-point correction $(\mathrm{RRHO})=$

Thermal correction to Energy $(\mathrm{RRHO})=$

Thermal correction to Enthalpy $(\mathrm{RRHO})=$

0.983779 (Hartree/Particle)

1.042875

1.043819

Thermal correction to Gibbs Free Energy $(\mathrm{RRHO})=0.883672$

Sum of electronic and zero-point Energies $(\mathrm{RRHO})=\quad-3121.931149$ 
Sum of electronic and thermal Energies $(\mathrm{RRHO})=$

Sum of electronic and thermal Enthalpies $(\mathrm{RRHO})=$

Sum of electronic and thermal Free Energies $(\mathrm{RRHO})=$
$-3121.872053$

$-3121.871109$

$-3122.031256$

Quasi-RRHO Thermochemistry ( $\mathrm{T}=298, \mathrm{v} 0=100 \mathrm{~cm}-1)$

Zero-point correction $(\mathrm{QRRHO})=$

Thermal correction to Energy $(\mathrm{QRRHO})=$

Thermal correction to Enthalpy (QRRHO)=

Thermal correction to Gibbs Free Energy (QRRHO)=

Sum of electronic and zero-point Energies (QRRHO)=

Sum of electronic and thermal Energies $(\mathrm{QRRHO})=$

Sum of electronic and thermal Enthalpies $(\mathrm{QRRHO})=$

Sum of electronic and thermal Free Energies (QRRHO)=
0.983779 (Hartree/Particle)

1.042875

1.043819

0.900819

$-3121.931149$

$-3121.872053$

$-3121.871109$

$-3122.014109$

Quasi-harmonic Thermochemistry $(T=298$, cutoff $=100 \mathrm{~cm}-1)$

Zero-point correction $(\mathrm{QHO})=$

Thermal correction to Energy $(\mathrm{QHO})=$

Thermal correction to Enthalpy $(\mathrm{QHO})=$

Thermal correction to Gibbs Free Energy $(\mathrm{QHO})=$

Sum of electronic and zero-point Energies $(\mathrm{QHO})=$

Sum of electronic and thermal Energies $(\mathrm{QHO})=$

Sum of electronic and thermal Enthalpies $(\mathrm{QHO})=$

Sum of electronic and thermal Free Energies $(\mathrm{QHO})=$

$\begin{array}{lrrr}\mathrm{C} & 3.6317590 & -0.1983350 & 1.2227850 \\ \mathrm{C} & 3.9945210 & -0.6576970 & -0.0508000 \\ \mathrm{C} & 5.3345370 & -0.9979280 & -0.2742720 \\ \mathrm{C} & 6.2784920 & -0.8932470 & 0.7410640 \\ \mathrm{C} & 5.9020230 & -0.4485920 & 2.0068720 \\ \mathrm{C} & 4.5740530 & -0.0995520 & 2.2429280 \\ \mathrm{C} & 2.9824820 & -0.8479800 & -1.1231740 \\ \mathrm{C} & 1.8797020 & 0.0574280 & -1.3069690 \\ \mathrm{C} & 0.8427430 & -0.3226000 & -2.1512510 \\ \mathrm{C} & 0.9277550 & -1.5109900 & -2.9430710 \\ \mathrm{C} & 2.0982790 & -2.3054290 & -2.8680370 \\ \mathrm{C} & 3.0844790 & -1.9741280 & -1.9074060 \\ \mathrm{C} & 2.2029950 & -3.4519170 & -3.6978680 \\ \mathrm{C} & 1.1787750 & -3.8007740 & -4.5424690 \\ \mathrm{C} & -0.0032070 & -3.0209620 & -4.5908930 \\ \mathrm{C} & -0.1253400 & -1.8972910 & -3.8098350 \\ \mathrm{H} & -0.8105640 & -3.3113200 & -5.2560960 \\ \mathrm{C} & 1.8452120 & 1.4252620 & -0.7139060 \\ \mathrm{C} & 0.6802360 & 1.8612050 & -0.1024680 \\ \mathrm{C} & 0.5883110 & 3.1427580 & 0.5200500 \\ \mathrm{C} & 1.7208480 & 3.9937330 & 0.5111720 \\ \mathrm{C} & 2.8927920 & 3.5558550 & -0.1531140 \\ \mathrm{C} & 2.9675840 & 2.3251890 & -0.7674240\end{array}$

0.983779 (Hartree/Particle)

1.043201

1.043819

0.905115

$-3121.931149$

$-3121.871727$

$-3121.871109$

$-3122.009813$
C $\quad-0.6106570$

C $\quad-0.6760140$

C $\quad 0.4555970$

C $\quad 1.6234580$

$\mathrm{H} \quad-1.4676360$

O -0.3915030

B -1.1315900

O -0.2564400

C $\quad 4.2248720$

C $\quad 5.4623210$

C $\quad 6.6514190$

C $\quad 6.6219370$

C $\quad 5.3952790$

C $\quad 4.2064140$

O -1.6094260

B $\quad-2.9246390$

O -3.9023480

B $\quad-3.5156680$

O $\quad-4.5688500$

C $\quad-4.4679390$

C $\quad-5.4887910$

C $\quad-5.4874790$

C $\quad-4.4719710$
3.5726990

4.8072140

5.6594780

5.2628830

2.9061830

1.0542080

0.5538390

0.4425190

1.9979500

2.2567250

1.9854290

1.4468590

1.1905220

1.4661340

$-0.7827570$

$-1.1061450$

$-0.2074170$

1.0851530

1.9440550

3.2841870

3.8437090

5.2085810

6.0226450
1.1436300

1.7431610

1.7437390

1.1405150

1.1224230

0.0275130

$-1.1439180$

$-2.3048800$

$-1.4950780$

$-0.8943050$

$-1.5630920$

$-2.8472900$

$-3.4582730$

$-2.7899820$

$-0.7010940$

$-0.6880280$

$-1.0294810$

$-1.3722770$

$-1.5911930$

$-1.8571140$

$-2.6251640$

$-2.8926220$

$-2.3946310$ 


\begin{tabular}{|c|c|c|c|}
\hline C & -3.4584180 & 5.4553500 & -1.6260600 \\
\hline C & -3.4478980 & 4.0907120 & -1.3503340 \\
\hline $\mathrm{O}$ & -3.2137340 & -2.4076770 & -0.3235650 \\
\hline C & -4.4321320 & -3.0262360 & -0.2485300 \\
\hline C & -5.6450530 & -2.3511130 & -0.0917550 \\
\hline C & -6.8224510 & -3.0898980 & 0.0114330 \\
\hline C & -6.8056750 & -4.4811630 & -0.0357680 \\
\hline C & -5.5890170 & -5.1437720 & -0.1873390 \\
\hline C & -4.4062320 & -4.4212000 & -0.29179 \\
\hline $\mathrm{O}$ & -2.2279700 & 1.4385460 & -1.47825 \\
\hline $\mathrm{N}$ & 0.0756300 & -1.1142010 & 1.646363 \\
\hline C & 0.9721040 & -2.0292990 & 1.7637 \\
\hline C & 1.2060980 & -3.0913260 & 0.8073500 \\
\hline C & 2.3737420 & -3.8508190 & 0.96884 \\
\hline C & 2.6324080 & -4.9096140 & 0.1090210 \\
\hline C & 1.7179270 & -5.2187440 & -0.8971930 \\
\hline C & 0.5516700 & -4.4680930 & -1.055501 \\
\hline C & 0.2903900 & -3.3991240 & -0.2127750 \\
\hline C & -0.2519280 & -0.0698700 & 2.6461710 \\
\hline $\mathrm{C}$ & 0.4752390 & -0.2789850 & 3.9558540 \\
\hline C & 0.0688030 & -1.2604040 & 4.8646540 \\
\hline C & 0.7684970 & -1.4440390 & 6.0525030 \\
\hline C & 1.8714420 & -0.6416800 & 6.3463320 \\
\hline C & 2.2732390 & 0.3442400 & 5.4498890 \\
\hline C & 1.5780720 & 0.5221480 & 4.2545930 \\
\hline C & -1.7623120 & 0.0088090 & 2.7774870 \\
\hline C & -2.3277820 & 1.2164770 & 3.1930990 \\
\hline C & -3.7070330 & 1.3386940 & 3.3182360 \\
\hline C & -4.5346100 & 0.2525070 & 3.0331990 \\
\hline C & -3.9749500 & -0.9572150 & 2.634770 \\
\hline C & -2.5909150 & -1.0806860 & 2.509867 \\
\hline $\mathrm{H}$ & -5.6629360 & -1.2694140 & -0.0579820 \\
\hline $\mathrm{H}$ & -7.7642830 & -2.5625390 & 0.1297750 \\
\hline $\mathrm{H}$ & -7.7301980 & -5.0434940 & 0.044735 \\
\hline $\mathrm{H}$ & -5.5581420 & -6.2284520 & -0.224856 \\
\hline $\mathrm{H}$ & -3.4474800 & -4.9165570 & -0.409171 \\
\hline $\mathrm{H}$ & -6.2722130 & 3.1930670 & -3.000008 \\
\hline $\mathrm{H}$ & -6.2850750 & 5.6356810 & -3.493132 \\
\hline $\mathrm{H}$ & -4.4705650 & 7.0874250 & -2.6045 \\
\hline
\end{tabular}

$\begin{array}{rrrr}\mathrm{H} & -2.6586520 & 6.0754010 & -1.2312340 \\ \mathrm{H} & -2.6487110 & 3.6529690 & -0.7658280 \\ \mathrm{H} & 0.3932710 & 6.6335030 & 2.2193600 \\ \mathrm{H} & 2.4910250 & 5.9176680 & 1.1307510 \\ \mathrm{H} & 3.7386590 & 4.2350600 & -0.2188670 \\ \mathrm{H} & 5.4855670 & 2.6364810 & 0.1238050 \\ \mathrm{H} & 7.6013270 & 2.1803470 & -1.0740500 \\ \mathrm{H} & 7.5481910 & 1.2290630 & -3.3705570 \\ \mathrm{H} & 5.3623070 & 0.7795210 & -4.4628020 \\ \mathrm{H} & 3.2537190 & 1.2672870 & -3.2725100 \\ \mathrm{H} & 5.6428640 & -1.3019170 & -1.2705560 \\ \mathrm{H} & 7.3151000 & -1.1458670 & 0.5385950 \\ \mathrm{H} & 6.6385500 & -0.3659650 & 2.8002040 \\ \mathrm{H} & 4.2674470 & 0.2485750 & 3.2253130 \\ \mathrm{H} & 2.6033700 & 0.1029340 & 1.4065360 \\ \mathrm{H} & 3.9084480 & -2.6656450 & -1.7527250 \\ \mathrm{H} & 3.1055040 & -4.0559150 & -3.6396950 \\ \mathrm{H} & 1.2682950 & -4.6795360 & -5.1743520 \\ \mathrm{H} & 0.0909270 & 0.8696120 & 2.2034580 \\ \mathrm{H} & -0.4766200 & -1.0319370 & 0.7701480 \\ \mathrm{H} & 1.5799590 & -2.0064100 & 2.6669760 \\ \mathrm{H} & 3.0768300 & -3.5947910 & 1.7572020 \\ \mathrm{H} & 3.5392810 & -5.4935860 & 0.2239950 \\ \mathrm{H} & 1.9169370 & -6.0469110 & -1.5707470 \\ \mathrm{H} & -0.1416480 & -4.6984960 & -1.8581120 \\ \mathrm{H} & -0.6180060 & -2.8182100 & -0.3475570 \\ \mathrm{H} & -0.8041540 & -1.8698280 & 4.6432970 \\ \mathrm{H} & 1.8837330 & 1.2966910 & 3.5531860 \\ \mathrm{H} & -2.1726280 & -2.0284870 & 2.1802030 \\ \mathrm{H} & -1.6809200 & 2.0646490 & 3.4055180 \\ \mathrm{H} & -1.5988590 & 5.1367350 & 2.2115040 \\ \mathrm{H} & -1.0149300 & -1.2781780 & -3.8456250 \\ \mathrm{H} & -4.1377490 & 2.2850570 & 3.6295500 \\ \mathrm{H} & -5.6118850 & 0.3512870 & 3.1201090 \\ \mathrm{H} & -4.6103000 & -1.8090950 & 2.4127970 \\ \mathrm{H} & 3.1224800 & 0.9800800 & 5.6803400 \\ \mathrm{H} & 2.4106090 & -0.7812010 & 7.2778790 \\ \mathrm{H} & 0.4477890 & -2.2062550 & 6.7553330\end{array}$




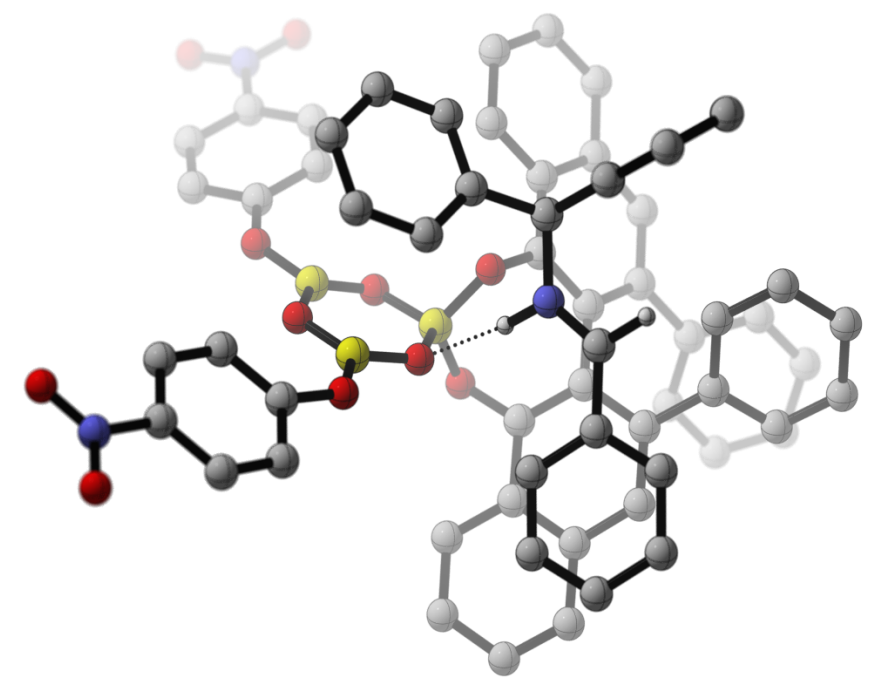

Boroxinate-H-Iminium Complex 4d

RRHO Thermochemistry $(T=298.15)$

Zero-point correction $(\mathrm{RRHO})=$

Thermal correction to Energy $(\mathrm{RRHO})=$

Thermal correction to Enthalpy $(\mathrm{RRHO})=$

1.147523 (Hartree/Particle)

1.208703

1.209647

Thermal correction to Gibbs Free Energy $(\mathrm{RRHO})=$

1.046810

Sum of electronic and zero-point Energies $(\mathrm{RRHO})=$ $-3530.631429$

Sum of electronic and thermal Energies (RRHO)= $-3530.570249$

Sum of electronic and thermal Enthalpies $(\mathrm{RRHO})=$ $-3530.569305$

Sum of electronic and thermal Free Energies $(\mathrm{RRHO})=\quad-3530.732142$

Quasi-RRHO Thermochemistry $(T=298.15$, v0 $=100 \mathrm{~cm}-1)$

Zero-point correction (QRRHO)=

1.147523 (Hartree/Particle)

Thermal correction to Energy $(\mathrm{QRRHO})=$

Thermal correction to Enthalpy (QRRHO)=

1.208703

1.209647

Thermal correction to Gibbs Free Energy $(\mathrm{QRRHO})=\quad 1.063021$

Sum of electronic and zero-point Energies (QRRHO)=

Sum of electronic and thermal Energies (QRRHO)=

$-3530.631429$

$-3530.570249$

Sum of electronic and thermal Enthalpies (QRRHO)= $-3530.569305$

Sum of electronic and thermal Free Energies $(\mathrm{QRRHO})=\quad-3530.715931$

Quasi-harmonic Thermochemistry $(T=298.15$, cutoff $=100 \mathrm{~cm}-1)$

Zero-point correction $(\mathrm{QHO})=$

Thermal correction to Energy $(\mathrm{QHO})=$

1.147523 (Hartree/Particle)

Thermal correction to Enthalpy $(\mathrm{QHO})=$

1.209009

Thermal correction to Gibbs Free Energy $(\mathrm{QHO})=$

1.209647

1.066733


Sum of electronic and zero-point Energies (QHO)= Sum of electronic and thermal Energies $(\mathrm{QHO})=$ Sum of electronic and thermal Enthalpies $(\mathrm{QHO})=$ Sum of electronic and thermal Free Energies $(\mathrm{QHO})=$
$-3530.631429$

$-3530.569943$

$-3530.569305$

$-3530.712219$

$\begin{array}{lrrr}\text { C } & 4.1431110 & -0.8235600 & 1.1098030 \\ \text { C } & 4.4334290 & -1.1298860 & -0.2268560 \\ \text { C } & 5.7337430 & -1.5423510 & -0.5439050 \\ \text { C } & 6.7083330 & -1.6571590 & 0.4408580 \\ \text { C } & 6.4026570 & -1.3647500 & 1.7684090 \\ \text { C } & 5.1159280 & -0.9447390 & 2.0984810 \\ \text { C } & 3.3853950 & -1.0874660 & -1.2807620 \\ \text { C } & 2.3605330 & -0.0776930 & -1.3085260 \\ \text { C } & 1.2845830 & -0.2482890 & -2.1715040 \\ \text { C } & 1.2546530 & -1.3158900 & -3.1221360 \\ \text { C } & 2.3521560 & -2.2093160 & -3.1852720 \\ \text { C } & 3.3760620 & -2.0991640 & -2.2136040 \\ \text { C } & 2.3449710 & -3.2358440 & -4.1652910 \\ \text { C } & 1.2828110 & -3.3774590 & -5.0229510 \\ \text { C } & 0.1731530 & -2.5005840 & -4.9356220 \\ \text { C } & 0.1601970 & -1.4885280 & -4.0066670 \\ \text { H } & -0.6647420 & -2.6269490 & -5.6142610 \\ \text { C } & 2.4482290 & 1.1923010 & -0.5310330 \\ \text { C } & 1.3318250 & 1.6305730 & 0.1625890 \\ \text { C } & 1.3457560 & 2.8236460 & 0.9450170 \\ \text { C } & 2.5397580 & 3.5828620 & 1.0151950 \\ \text { C } & 3.6653340 & 3.1462070 & 0.2745480 \\ \text { C } & 3.6372880 & 2.0036390 & -0.4942490 \\ \text { C } & 0.1914340 & 3.2605680 & 1.6438570 \\ \text { C } & 0.2279080 & 4.4139940 & 2.3895570 \\ \text { C } & 1.4208450 & 5.1741430 & 2.4679910 \\ \text { C } & 2.5474020 & 4.7686700 & 1.7961670 \\ \text { H } & -0.7140970 & 2.6667620 & 1.5616100 \\ \text { O } & 0.1991040 & 0.8948580 & 0.2097470 \\ \text { B } & -0.5891530 & 0.6370060 & -1.0026860 \\ \text { O } & 0.2504400 & 0.6200060 & -2.1881120 \\ \text { C } & 4.8558700 & 1.6809840 & -1.2865690 \\ \text { C } & 6.1160240 & 1.7613780 & -0.6827190 \\ \text { C } & 7.2726560 & 1.4894980 & -1.4058260 \\ \text { C } & 7.1870280 & 1.1297170 & -2.7486280 \\ \text { C } & 5.9376570 & 1.0532880 & -3.3625830 \\ \text { C } & 4.7813890 & 1.3292840 & -2.6395150 \\ \text { O } & -1.1890380 & -0.7019590 & -0.7469590 \\ \text { B } & -2.5225110 & -0.8941760 & -0.7179490 \\ \text { O } & -3.4226780 & 0.1301870 & -0.8685050 \\ \text { B } & -2.9168120 & 1.4124440 & -1.0455380 \\ \text { O } & -3.8930780 & 2.3927600 & -1.0801330 \\ \text { C } & -3.6966970 & 3.7357600 & -1.0907580 \\ \text { C } & -4.7681780 & 4.5065650 & -1.5565940 \\ & -4.6857770 & 5.8885570 & -1.5553300\end{array}$

\begin{tabular}{|c|c|c|c|}
\hline C & -3.5230060 & 6.4861170 & -1.0798970 \\
\hline C & -2.4483070 & 5.7383280 & -0.6137150 \\
\hline C & -2.5320490 & 4.3537370 & -0.6194490 \\
\hline O & -2.9383610 & -2.2089760 & -0.5494180 \\
\hline C & -4.1964440 & -2.7094610 & -0.4845260 \\
\hline C & -5.3357040 & -1.9430890 & -0.2046550 \\
\hline C & -6.5724670 & -2.5684440 & -0.1239220 \\
\hline C & -6.6572420 & -3.9411910 & -0.3188250 \\
\hline C & -5.5363840 & -4.7173490 & -0.593890 \\
\hline C & -4.3037380 & -4.0929720 & -0.674068 \\
\hline $\mathrm{O}$ & -1.6113010 & 1.6579160 & -1.17677 \\
\hline $\mathrm{N}$ & 0.5657500 & -1.4834880 & 1.5127930 \\
\hline C & 1.3760950 & -2.4831010 & 1.4895820 \\
\hline C & 1.5072200 & -3.4180560 & 0.3919590 \\
\hline C & 2.6080530 & -4.2862530 & 0.4155780 \\
\hline C & 2.7608230 & -5.2327370 & -0.588572 \\
\hline C & 1.8069190 & -5.3230690 & -1.6015040 \\
\hline C & 0.7061590 & -4.4645140 & -1.6227930 \\
\hline C & 0.5518610 & -3.5052350 & -0.6346210 \\
\hline C & 0.3179820 & -0.5723500 & 2.6556760 \\
\hline C & 1.0388680 & -1.0198910 & 3.9067600 \\
\hline C & 0.5643410 & -2.0939090 & 4.664830 \\
\hline C & 1.2580360 & -2.5090470 & 5.7964880 \\
\hline C & 2.4236810 & -1.8473120 & 6.1834470 \\
\hline C & 2.8945960 & -0.7707540 & 5.4366860 \\
\hline C & 2.2050630 & -0.3603640 & 4.2966890 \\
\hline C & -1.1833870 & -0.4221970 & 2.8227210 \\
\hline C & -1.6736140 & 0.7537730 & 3.3950120 \\
\hline C & -3.0419940 & 0.9346590 & 3.5635710 \\
\hline C & -3.9337350 & -0.0628740 & 3.1693530 \\
\hline C & -3.4484570 & -1.2441780 & 2.6171700 \\
\hline C & -2.0760020 & -1.4244510 & 2.4433970 \\
\hline $\mathrm{H}$ & -5.2496870 & -0.8752610 & -0.0559050 \\
\hline $\mathrm{H}$ & -7.4702740 & -2.0018160 & 0.0904030 \\
\hline $\mathrm{H}$ & -5.6417010 & -5.7852560 & -0.738980 \\
\hline $\mathrm{H}$ & -3.4015980 & -4.6568990 & -0.8845490 \\
\hline $\mathrm{H}$ & -5.6572320 & 3.9982030 & -1.9123970 \\
\hline $\mathrm{H}$ & -5.5019190 & 6.5046360 & -1.9115860 \\
\hline $\mathrm{H}$ & -1.5583020 & 6.2382760 & -0.2508020 \\
\hline $\mathrm{H}$ & -1.6977340 & 3.7572450 & -0.2762750 \\
\hline $\mathrm{H}$ & 1.4379050 & 6.0857120 & 3.0571810 \\
\hline $\mathrm{H}$ & 3.4613970 & 5.3546630 & 1.8456200 \\
\hline $\mathrm{H}$ & 4.5598620 & 3.7633440 & 0.2757520 \\
\hline $\mathrm{H}$ & 6.1802330 & 2.0004270 & 0.3755120 \\
\hline $\mathrm{H}$ & 8.2399770 & 1.5436490 & -0.915185 \\
\hline
\end{tabular}




$\begin{array}{rrrrrrrr}\mathrm{H} & 8.0873980 & 0.9120380 & -3.3152280 & \mathrm{H} & -0.3544070 & -2.5955840 & 4.3676870 \\ \mathrm{H} & 5.8617640 & 0.7837910 & -4.4117150 & \mathrm{H} & 2.5640150 & 0.4859580 & 3.7133920 \\ \mathrm{H} & 3.8114900 & 1.2727600 & -3.1254470 & \mathrm{H} & -1.7135450 & -2.3487570 & 1.9996690 \\ \mathrm{H} & 5.9900970 & -1.7262440 & -1.5833620 & \mathrm{H} & -0.9767850 & 1.5316200 & 3.6984050 \\ \mathrm{H} & 7.7144280 & -1.9612730 & 0.1678370 & \mathrm{H} & -0.6606700 & 4.7514680 & 2.9150220 \\ \mathrm{H} & 7.1630720 & -1.4534400 & 2.5381320 & \mathrm{H} & -0.6719120 & -0.7966500 & -3.9365260 \\ \mathrm{H} & 4.8661300 & -0.7123430 & 3.1298270 & \mathrm{H} & -3.4136470 & 1.8567350 & 3.9989710 \\ \mathrm{H} & 3.1498810 & -0.4672740 & 1.3714700 & \mathrm{H} & -5.0020920 & 0.0802220 & 3.2959320 \\ \mathrm{H} & 4.1391750 & -2.8720400 & -2.1770700 & \mathrm{H} & -4.1347440 & -2.0314920 & 2.3212570 \\ \mathrm{H} & 3.1921220 & -3.9160320 & -4.2130910 & \mathrm{H} & 3.7938080 & -0.2451040 & 5.7427530 \\ \mathrm{H} & 1.2865820 & -4.1652010 & -5.7704600 & \mathrm{H} & 2.9590430 & -2.1674880 & 7.0715510 \\ \mathrm{H} & 0.7168870 & 0.3974640 & 2.3442830 & \mathrm{H} & 0.8851540 & -3.3424590 & 6.3830600 \\ \mathrm{H} & 0.0246490 & -1.2264620 & 0.6680570 & \mathrm{~N} & -3.4325320 & 7.9442120 & -1.0662220 \\ \mathrm{H} & 1.9841010 & -2.6402280 & 2.3790610 & \mathrm{~N} & -7.9633110 & -4.5915390 & -0.2268610 \\ \mathrm{H} & 3.3428650 & -4.2023060 & 1.2120130 & \mathrm{O} & -8.0107500 & -5.7990010 & -0.3923870 \\ \mathrm{H} & 3.6161110 & -5.8997850 & -0.5802940 & \mathrm{O} & -8.9305840 & -3.8948880 & 0.0295210 \\ \mathrm{H} & 1.9234700 & -6.0634240 & -2.3872090 & \mathrm{O} & -4.3867050 & 8.5794170 & -1.4845020 \\ \mathrm{H} & -0.0169510 & -4.5234780 & -2.4301040 & \mathrm{O} & -2.4094170 & 8.4495580 & -0.6352330 \\ \mathrm{H} & -0.3053990 & -2.8380420 & -0.6634330 & & & & \end{array}$

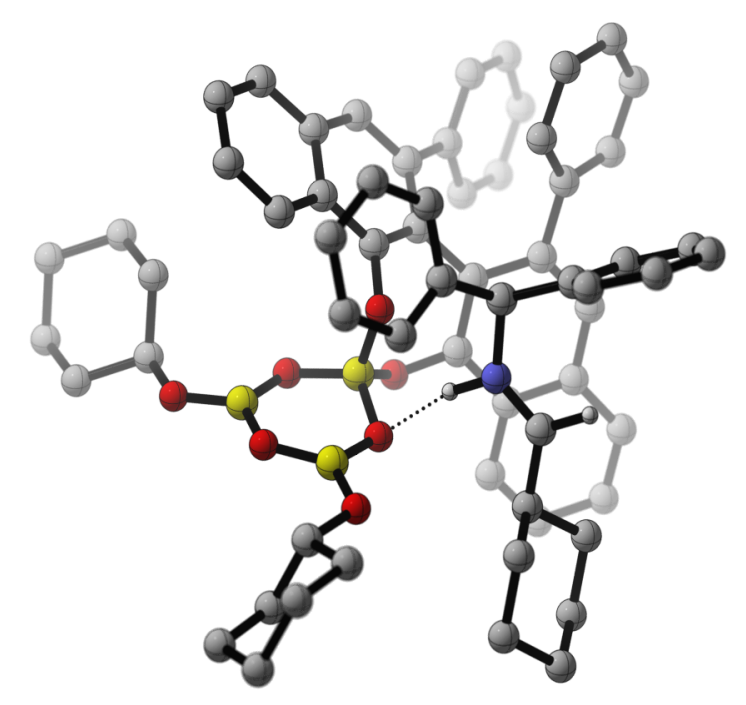

Boroxinate-H-Iminium Complex 4e

File Name: catim4e

RRHO Thermochemistry $(T=298)$

Zero-point correction $(\mathrm{RRHO})=$

Thermal correction to Energy $(\mathrm{RRHO})=$

Thermal correction to Enthalpy $(\mathrm{RRHO})=$

1.197594 (Hartree/Particle)

1.260170

1.261114

Thermal correction to Gibbs Free Energy $(\mathrm{RRHO})=1.096845$

Sum of electronic and zero-point Energies $(\mathrm{RRHO})=\quad-3132.564481$ 
Sum of electronic and thermal Energies $(\mathrm{RRHO})=$

Sum of electronic and thermal Enthalpies $(\mathrm{RRHO})=$

Sum of electronic and thermal Free Energies $(\mathrm{RRHO})=$
$-3132.501905$

$-3132.500961$

$-3132.665230$

Quasi-RRHO Thermochemistry ( $\mathrm{T}=298, \mathrm{v} 0=100 \mathrm{~cm}-1)$

Zero-point correction $(\mathrm{QRRHO})=$

Thermal correction to Energy $(\mathrm{QRRHO})=$

Thermal correction to Enthalpy (QRRHO)=

Thermal correction to Gibbs Free Energy $(\mathrm{QRRHO})=$

Sum of electronic and zero-point Energies (QRRHO)=

Sum of electronic and thermal Energies $(\mathrm{QRRHO})=$

Sum of electronic and thermal Enthalpies $(\mathrm{QRRHO})=$

Sum of electronic and thermal Free Energies (QRRHO)=
1.197594 (Hartree/Particle)

1.260170

1.261114

1.113037

$-3132.564481$

$-3132.501905$

$-3132.500961$

$-3132.649038$

Quasi-harmonic Thermochemistry $(T=298$, cutoff $=100 \mathrm{~cm}-1)$

Zero-point correction $(\mathrm{QHO})=$

Thermal correction to Energy $(\mathrm{QHO})=$

Thermal correction to Enthalpy $(\mathrm{QHO})=$

Thermal correction to Gibbs Free Energy $(\mathrm{QHO})=$

Sum of electronic and zero-point Energies $(\mathrm{QHO})=$

Sum of electronic and thermal Energies $(\mathrm{QHO})=$

Sum of electronic and thermal Enthalpies $(\mathrm{QHO})=$

Sum of electronic and thermal Free Energies $(\mathrm{QHO})=$
1.197594 (Hartree/Particle)

1.260497

1.261114

1.116060

$-3132.564481$

$-3132.501578$

$-3132.500961$

$-3132.646015$

$\begin{array}{lrrr}\text { C } & 4.0065340 & -0.8522870 & 1.4627390 \\ \text { C } & 4.2045610 & -1.2995620 & 0.1496420 \\ \text { C } & 5.4795150 & -1.7524910 & -0.2116340 \\ \text { C } & 6.5236790 & -1.7602080 & 0.7068720 \\ \text { C } & 6.3129770 & -1.3150470 & 2.0094150 \\ \text { C } & 5.0494350 & -0.8618480 & 2.3834360 \\ \text { C } & 3.1040720 & -1.3393270 & -0.8556830 \\ \text { C } & 2.1651440 & -0.2635630 & -1.0238040 \\ \text { C } & 1.1304480 & -0.4123280 & -1.9433880 \\ \text { C } & 1.0294490 & -1.5775520 & -2.7682840 \\ \text { C } & 1.9949760 & -2.6067970 & -2.6282970 \\ \text { C } & 2.9996540 & -2.4701600 & -1.6387080 \\ \text { C } & 1.9268140 & -3.7314170 & -3.4942350 \\ \text { C } & 0.9574780 & -3.8113130 & -4.4636900 \\ \text { C } & -0.0080750 & -2.7827720 & -4.5950500 \\ \text { C } & 0.0178760 & -1.6944730 & -3.7563980 \\ \text { H } & -0.7717150 & -2.8599330 & -5.3632330 \\ \text { C } & 2.2457340 & 1.0108520 & -0.2522040 \\ \text { C } & 1.0953230 & 1.4257270 & 0.3995210 \\ \text { C } & 1.0843240 & 2.5496010 & 1.2753170 \\ \text { C } & 2.2690630 & 3.3165740 & 1.4071050 \\ \text { C } & 3.4203740 & 2.9278280 & 0.6761510\end{array}$

$\begin{array}{lr}\text { C } & 3.4320480 \\ \text { C } & -0.0780500 \\ \text { C } & -0.0628570 \\ \text { C } & 1.1058560 \\ \text { C } & 2.2440720 \\ \text { H } & -0.9644070 \\ \text { O } & -0.0590830 \\ \text { B } & -0.7067790 \\ \text { O } & 0.2327340 \\ \text { C } & 4.6762360 \\ \text { C } & 5.9172540 \\ \text { C } & 7.0932100 \\ \text { C } & 7.0476090 \\ \text { C } & 5.8181500 \\ \text { C } & 4.6419090 \\ \text { O } & -1.8173480 \\ \text { B } & -3.0144400 \\ \text { O } & -3.3161910 \\ \text { B } & -2.4009800 \\ \text { O } & -2.8344640 \\ \text { O } & -3.9223630 \\ \text { O } & -1.1754630\end{array}$

1.8116670

$-0.1312470$

2.9055670

2.0035290

4.0023720

2.8287460

4.7953790

2.9401700

4.4606490

2.2480570

2.2850900

1.9008520

0.7446250

0.2377450

0.9060760

$-1.0932210$

0.5658510

$-2.1554960$

1.4966230

$-0.8823620$

$-0.2400540$

$-0.9236710$

$-2.2643000$

$-2.9172670$

$-2.2338310$

$-1.0261540$

$-0.5324280$

$-0.4072720$

$-0.8839890$

$-0.9188480$

$-0.1478290$

$-1.3095180$ 


\begin{tabular}{|c|c|c|c|c|c|c|c|}
\hline $\mathrm{N}$ & -0.6575860 & -2.0517990 & 0.6050720 & $\mathrm{H}$ & -2.1316810 & -2.4206810 & -1.4145170 \\
\hline C & -1.0657520 & -3.2027500 & 0.2272270 & C & -2.4991270 & -4.6149130 & -3.0232570 \\
\hline $\mathrm{C}$ & 0.2065880 & -1.7437630 & 1.7648370 & $\mathrm{H}$ & -1.3632960 & -5.3954410 & -1.354443 \\
\hline C & 0.7143450 & -3.0072410 & 2.4293570 & $\mathrm{H}$ & -0.5138490 & -4.1461010 & -2.278056 \\
\hline C & 0.0331640 & -3.5900350 & 3.5003770 & C & -4.3801350 & -3.9876270 & -1.453336 \\
\hline C & 0.4993980 & -4.7759480 & 4.0620690 & $\mathrm{H}$ & -3.2505370 & -4.7637890 & 0.223777 \\
\hline C & 1.6478530 & -5.3839440 & 3.5589090 & $\mathrm{H}$ & -3.7216490 & -3.0587130 & 0.392490 \\
\hline C & 2.3286940 & -4.8070180 & 2.4889120 & C & -3.8823740 & -4.9991980 & -2.490395 \\
\hline C & 1.8615040 & -3.6238580 & 1.9237260 & $\mathrm{H}$ & -2.1326480 & -5.3684110 & -3.7289 \\
\hline $\mathrm{C}$ & -0.5228900 & -0.7966170 & 2.7028260 & $\mathrm{H}$ & -2.5687520 & -3.6688570 & -3.57602 \\
\hline C & 0.2335140 & -0.1321700 & 3.6700780 & $\mathrm{H}$ & -5.3454910 & -4.3048250 & -1.043318 \\
\hline C & -0.3781900 & 0.7362970 & 4.5668150 & $\mathrm{H}$ & -4.5368140 & -3.0091240 & -1.927124 \\
\hline C & -1.7541460 & 0.9524750 & 4.5034020 & $\mathrm{H}$ & -4.5978510 & -5.0766460 & -3.315621 \\
\hline C & -2.5109660 & 0.2934430 & 3.5394470 & $\mathrm{H}$ & -3.8258630 & -5.9921150 & -2.023050 \\
\hline C & -1.8998090 & -0.5836860 & 2.6428090 & $\mathrm{C}$ & -1.9759780 & 4.9370310 & -1.43290 \\
\hline $\mathrm{H}$ & 1.1004500 & 5.6707250 & 3.5830000 & C & -2.8498280 & 6.1284930 & -1.80583 \\
\hline $\mathrm{H}$ & 3.1441620 & 5.0635850 & 2.3374140 & $\mathrm{C}$ & -0.9177840 & 5.3280040 & -0.406267 \\
\hline $\mathrm{H}$ & 4.3078880 & 3.5529650 & 0.7321050 & $\mathrm{H}$ & -1.4701020 & 4.5584320 & -2.332737 \\
\hline $\mathrm{H}$ & 5.9500450 & 1.8246870 & 0.8173290 & $\mathrm{C}$ & -2.0014590 & 7.2979100 & -2.311816 \\
\hline $\mathrm{H}$ & 8.0449730 & 1.3373170 & -0.4027700 & $\mathrm{H}$ & -3.4072570 & 6.4327440 & -0.909345 \\
\hline $\mathrm{H}$ & 7.9638130 & 0.6787430 & -2.7985880 & $\mathrm{H}$ & -3.5865470 & 5.8197890 & -2.555627 \\
\hline $\mathrm{H}$ & 5.7737590 & 0.5605740 & -3.9657640 & C & -0.0712340 & 6.5030220 & -0.901575 \\
\hline $\mathrm{H}$ & 3.6872720 & 1.0835780 & -2.7494250 & $\mathrm{H}$ & -1.4310610 & 5.6013130 & 0.526561 \\
\hline $\mathrm{H}$ & 5.6596380 & -2.0564980 & -1.2387450 & $\mathrm{H}$ & -0.2853900 & 4.4618890 & -0.190017 \\
\hline $\mathrm{H}$ & 7.5086360 & -2.0971610 & 0.3975820 & $\mathrm{C}$ & -0.9473250 & 7.7006510 & -1.276730 \\
\hline $\mathrm{H}$ & 7.1277140 & -1.3151520 & 2.7270900 & $\mathrm{H}$ & -2.6448520 & 8.1494460 & -2.558418 \\
\hline $\mathrm{H}$ & 4.8730190 & -0.5123520 & 3.3963390 & $\mathrm{H}$ & -1.4958510 & 7.0032070 & -3.242000 \\
\hline $\mathrm{H}$ & 3.0334700 & -0.4746040 & 1.7642530 & $\mathrm{H}$ & 0.6591690 & 6.7791690 & -0.133113 \\
\hline $\mathrm{H}$ & 3.7039920 & -3.2859940 & -1.4960130 & $\mathrm{H}$ & 0.5032510 & 6.1872480 & -1.783371 \\
\hline $\mathrm{H}$ & 2.6693070 & -4.5181890 & -3.3868380 & $\mathrm{H}$ & -0.3313570 & 8.5217830 & -1.660225 \\
\hline $\mathrm{H}$ & 0.9253870 & -4.6665400 & -5.1322250 & $\mathrm{H}$ & -1.4538790 & 8.0737720 & -0.375569 \\
\hline $\mathrm{H}$ & 1.0508960 & -1.2033250 & 1.3209880 & C & -5.2015030 & -0.1988680 & 0.338975 \\
\hline $\mathrm{H}$ & -0.9860160 & -1.2229540 & 0.0625750 & $\mathrm{C}$ & -6.1287350 & 0.1522510 & -0.820453 \\
\hline $\mathrm{H}$ & -0.7267560 & -4.0708330 & 0.7942630 & C & -5.7826610 & -1.3311320 & 1.175654 \\
\hline $\mathrm{H}$ & -0.8539770 & -3.1067280 & 3.9003540 & $\mathrm{H}$ & -5.0751080 & 0.6911540 & 0.971383 \\
\hline $\mathrm{H}$ & 2.3861180 & -3.1728130 & 1.0844880 & $\mathrm{C}$ & -7.5240030 & 0.5226160 & -0.309600 \\
\hline $\mathrm{H}$ & -2.5096480 & -1.0802480 & 1.8916450 & $\mathrm{H}$ & -6.1910940 & -0.7230590 & -1.482472 \\
\hline $\mathrm{H}$ & 1.3075800 & -0.2983630 & 3.7185750 & $\mathrm{H}$ & -5.6920330 & 0.9753130 & -1.395238 \\
\hline $\mathrm{H}$ & -0.9520260 & 4.2713800 & 3.3913520 & $\mathrm{C}$ & -7.1721470 & -0.9598170 & 1.701358 \\
\hline $\mathrm{H}$ & -0.7104930 & -0.8951170 & -3.8405380 & $\mathrm{H}$ & -5.8556610 & -2.2260870 & 0.541082 \\
\hline $\mathrm{H}$ & 0.2222810 & 1.2573030 & 5.3056890 & $\mathrm{H}$ & -5.0994300 & -1.5698840 & 2.000788 \\
\hline $\mathrm{H}$ & -2.2307550 & 1.6374070 & 5.1976290 & $\mathrm{C}$ & -8.1138010 & -0.5992920 & 0.548862 \\
\hline $\mathrm{H}$ & -3.5826530 & 0.4594260 & 3.4776890 & $\mathrm{H}$ & -8.1841750 & 0.7482420 & -1.153400 \\
\hline $\mathrm{H}$ & 3.2272330 & -5.2727800 & 2.0970870 & $\mathrm{H}$ & -7.4549810 & 1.4393930 & 0.291582 \\
\hline $\mathrm{H}$ & 2.0138060 & -6.3041770 & 4.0033850 & $\mathrm{H}$ & -7.5834360 & -1.7855700 & 2.291238 \\
\hline $\mathrm{H}$ & -0.0313020 & -5.2211140 & 4.8976530 & $\mathrm{H}$ & -7.0845010 & -0.0987270 & 2.378357 \\
\hline C & -2.0086010 & -3.3823840 & -0.9005290 & $\mathrm{H}$ & -9.0949590 & -0.3065670 & 0.937799 \\
\hline C & -1.4914490 & -4.4411180 & -1.8859500 & $\mathrm{H}$ & -8.2699980 & -1.4884040 & -0.077681 \\
\hline C & -3.3720400 & -3.8140980 & -0.3166460 & & & & \\
\hline
\end{tabular}




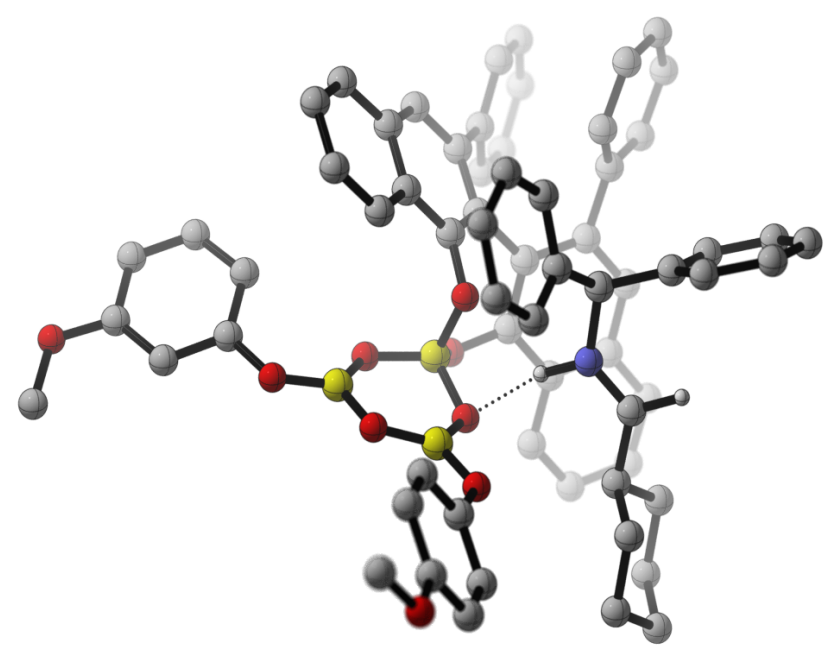

Boroxinate-H-Iminium Complex $4 \mathbf{f}$

File Name: catim4f

RRHO Thermochemistry $(T=298)$

Zero-point correction $(\mathrm{RRHO})=$

Thermal correction to Energy $(\mathrm{RRHO})=$

1.121538 (Hartree/Particle)

Thermal correction to Enthalpy $(\mathrm{RRHO})=$

1.186641

1.187584

Thermal correction to Gibbs Free Energy $(\mathrm{RRHO})=\quad 1.017359$

Sum of electronic and zero-point Energies (RRHO)= $\quad-3354.365852$

Sum of electronic and thermal Energies $(\mathrm{RRHO})=\quad-3354.300749$

Sum of electronic and thermal Enthalpies $(\mathrm{RRHO})=\quad-3354.299806$

Sum of electronic and thermal Free Energies $(\mathrm{RRHO})=\quad-3354.470031$

Quasi-RRHO Thermochemistry ( $T=298$, v0 = $100 \mathrm{~cm}-1)$

Zero-point correction (QRRHO)=

1.121538 (Hartree/Particle)

Thermal correction to Energy $(\mathrm{QRRHO})=$

1.186641

Thermal correction to Enthalpy $(\mathrm{QRRHO})=$

1.187584

Thermal correction to Gibbs Free Energy (QRRHO)=

1.034260

Sum of electronic and zero-point Energies $(\mathrm{QRRHO})=$ $-3354.365852$

Sum of electronic and thermal Energies $(Q R R H O)=$ $-3354.300749$

Sum of electronic and thermal Enthalpies (QRRHO)= $-3354.299806$

Sum of electronic and thermal Free Energies $(Q R R H O)=$

$-3354.453130$

Quasi-harmonic Thermochemistry $(T=298$, cutoff $=100 \mathrm{~cm}-1)$

Zero-point correction $(\mathrm{QHO})=$

1.121538 (Hartree/Particle) 
Thermal correction to Energy $(\mathrm{QHO})=$

Thermal correction to Enthalpy $(\mathrm{QHO})=$

Thermal correction to Gibbs Free Energy (QHO)=

Sum of electronic and zero-point Energies $(\mathrm{QHO})=$ Sum of electronic and thermal Energies $(\mathrm{QHO})=$ Sum of electronic and thermal Enthalpies $(\mathrm{QHO})=$ Sum of electronic and thermal Free Energies (QHO)=

$\begin{array}{lrrr}\text { C } & 4.2178940 & 0.0172990 & 1.7399500 \\ \text { C } & 4.6576220 & -0.1931010 & 0.4269190 \\ \text { C } & 6.0365120 & -0.2288300 & 0.1861800 \\ \text { C } & 6.9478830 & -0.0592830 & 1.2229610 \\ \text { C } & 6.4973660 & 0.1478670 & 2.5243340 \\ \text { C } & 5.1278630 & 0.1844810 & 2.7786870 \\ \text { C } & 3.7108480 & -0.4126860 & -0.7030450 \\ \text { C } & 2.5231790 & 0.3766480 & -0.8869240 \\ \text { C } & 1.6651240 & 0.0626700 & -1.9368720 \\ \text { C } & 1.9653140 & -0.9900550 & -2.8571520 \\ \text { C } & 3.1632510 & -1.7301460 & -2.6893330 \\ \text { C } & 4.0007380 & -1.4317520 & -1.5862780 \\ \text { C } & 3.4805650 & -2.7421030 & -3.6349910 \\ \text { C } & 2.6519160 & -2.9877050 & -4.7020440 \\ \text { C } & 1.4546280 & -2.2471810 & -4.8624140 \\ \text { C } & 1.1108210 & -1.2762700 & -3.9530650 \\ \text { H } & 0.8078320 & -2.4522460 & -5.7102010 \\ \text { C } & 2.1554390 & 1.5183880 & 0.0009240 \\ \text { C } & 0.8737490 & 1.5176390 & 0.5276880 \\ \text { C } & 0.4321250 & 2.5029720 & 1.4564690 \\ \text { C } & 1.3204150 & 3.5525640 & 1.8008980 \\ \text { C } & 2.6126070 & 3.5743160 & 1.2171490 \\ \text { C } & 3.0366360 & 2.6008230 & 0.3394990 \\ \text { C } & -0.8691620 & 2.4599500 & 2.0181230 \\ \text { C } & -1.2773030 & 3.4442780 & 2.8829570 \\ \text { C } & -0.4044250 & 4.5100920 & 3.2143540 \\ \text { C } & 0.8636670 & 4.5612730 & 2.6907300 \\ \text { H } & -1.5178800 & 1.6260000 & 1.7616820 \\ \text { O } & -0.0073220 & 0.5571560 & 0.1661560 \\ \text { B } & -0.5379930 & 0.6985590 & -1.2167540 \\ \text { O } & 0.5511060 & 0.7828290 & -2.1724040 \\ \text { C } & 4.3901840 & 2.7336340 & -0.2617010 \\ \text { C } & 5.4820680 & 3.0718750 & 0.5456320 \\ \text { C } & 6.7551370 & 3.2045820 & 0.0016640 \\ \text { C } & 6.9568410 & 3.0018550 & -1.3615800 \\ \text { C } & 5.8742330 & 2.6758060 & -2.1768890 \\ \text { C } & 4.6001770 & 2.5449400 & -1.6329630 \\ \text { O } & -1.3163960 & -0.5547610 & -1.3853540 \\ \text { B } & -2.5949470 & -0.5511210 & -0.9294420 \\ \text { O } & -3.2883270 & 0.6083160 & -0.6949430 \\ \text { B } & -2.6579030 & 1.8226870 & -0.9675130 \\ \text { O } & -3.4742530 & 2.9195650 & -0.8329010\end{array}$

1.186971

1.187584

1.037408

$-3354.365852$

$-3354.300419$

$-3354.299806$

$-3354.449982$

C $\quad-3.0885490$

C $\quad-4.0682220$

C $\quad-3.7831930$

C $\quad-2.5286820$

C $\quad-1.5756270$

C $\quad-1.8363970$

O -3.1627740

C $\quad-4.4011030$

C $\quad-4.8910720$

C -6.1244230

C $\quad-6.8685880$

C $\quad-6.3707500$

C $\quad-5.1477570$

O $\quad-1.3708350$

$\mathrm{N} \quad 0.1405840$

C $\quad 0.0425750$

C $\quad 0.8262870$

C $\quad 1.6229260$

C $\quad 1.0797010$

C $\quad 1.8237600$

C $\quad 3.1133330$

C $\quad 3.6587700$

C $\quad 2.9149260$

C -0.1661880

C $\quad 0.3460780$

C $\quad-0.4986450$

C -1.8676120

C $\quad-2.3825750$

C $\quad-1.5369300$

$\mathrm{H} \quad-4.3200380$

H -6.4889160

H -6.9646820

$\begin{array}{ll}H & -6.9646820 \\ H & -4.7590720\end{array}$

$\mathrm{H} \quad-5.0274300$

$\mathrm{H}-2.3319820$

$\mathrm{H}-0.6015020$

H $\quad-1.0845310$

H $\quad-0.7442640$

H 1.5360620

H 3.2694520

H $\quad 5.3328350$

H $\quad 7.5934730$

H $\quad 7.9508790$

H $\quad 6.0199250$
4.2285550

5.0934660

6.4515870

6.9430150

6.0642850

4.6995450

$-1.7915490$

$-2.0018820$

$-1.2331240$

$-1.5379330$

$-2.6195840$

$-3.3894880$

$-3.0820650$

1.8753090

$-2.3170320$

$-3.5045090$

$-1.8963460$

$-3.0342800$

$-3.8613940$

$-4.9236660$

$-5.1620890$

$-4.3389020$

$-3.2800330$

$-1.2596970$

$-0.4266180$

0.1470530

$-0.1133660$

$-0.9431200$

$-1.5128630$

$-0.3840620$

$-0.9178040$

$-4.2237250$

$-3.6698410$

4.6725950

8.0046220

6.4420190

4.0189730

5.2899900

5.3761670

4.4115420

3.1866560

3.4497030

3.0994150

2.5290590
$-0.9687940$

$-1.4647080$

$-1.5853540$

$-1.2078040$

$-0.7163000$

$-0.5882460$

$-0.7017550$

$-0.1399400$

0.9091760

1.4876660

1.0165290

$-0.0399860$

$-0.6140500$

$-1.3439990$

0.2917840

$-0.1718970$

1.5342580

2.1413280

3.1268020

3.6345730

3.1642100

2.1809780

1.6688510

2.4900890

3.4870160

4.4293160

4.3888630

3.3980790

2.4441310

1.2662040

2.2982370

$-0.3982210$

$-1.4400300$

$-1.7394780$

$-1.3101760$

$-0.4206220$

$-0.2104760$

3.8890130

2.9463770

1.4390660

1.6158220

0.6472350

$-1.7876690$

$-3.2429430$ 


$\begin{array}{rrrr}\mathrm{H} & 3.7576890 & 2.3006340 & -2.2733870 \\ \mathrm{H} & 6.3914620 & -0.3451050 & -0.8338320 \\ \mathrm{H} & 8.0125620 & -0.0707170 & 1.0093580 \\ \mathrm{H} & 7.2071020 & 0.2853360 & 3.3343730 \\ \mathrm{H} & 4.7644220 & 0.3441480 & 3.7895130 \\ \mathrm{H} & 3.1534210 & 0.0719210 & 1.9482510 \\ \mathrm{H} & 4.8920210 & -2.0349220 & -1.4312750 \\ \mathrm{H} & 4.3999840 & -3.3074300 & -3.5056110 \\ \mathrm{H} & 2.9112470 & -3.7527950 & -5.4278200 \\ \mathrm{H} & 1.5171780 & -1.1186070 & 1.1885280 \\ \mathrm{H} & -0.3051080 & -1.5475260 & -0.2485120 \\ \mathrm{H} & 0.5067840 & -4.3074770 & 0.4026890 \\ \mathrm{H} & 0.0800640 & -3.6647180 & 3.5044320 \\ \mathrm{H} & 3.3351100 & -2.6369230 & 0.8984550 \\ \mathrm{H} & -1.9598040 & -2.1502090 & 1.6700680 \\ \mathrm{H} & 1.4152280 & -0.2300530 & 3.5248660 \\ \mathrm{H} & -2.2739470 & 3.4121170 & 3.3123740 \\ \mathrm{H} & 0.1993120 & -0.6978220 & -4.0569820 \\ \mathrm{H} & -0.0899380 & 0.8063910 & 5.1883300 \\ \mathrm{H} & -2.5291240 & 0.3325950 & 5.1248170 \\ \mathrm{H} & -3.4460560 & -1.1589230 & 3.3621420 \\ \mathrm{H} & 4.6658150 & -4.5151530 & 1.8168290 \\ \mathrm{H} & 3.6945240 & -5.9855340 & 3.5672700 \\ \mathrm{H} & 1.3977910 & -5.5601680 & 4.4035600 \\ \mathrm{C} & -0.6556100 & -3.8273030 & -1.4378300 \\ \mathrm{C} & 0.3367150 & -4.5183960 & -2.3936110\end{array}$

$\begin{array}{lrrr}\mathrm{C} & -1.8516140 & -4.7532120 & -1.1380590 \\ \mathrm{H} & -1.0195280 & -2.8985850 & -1.8935020 \\ \mathrm{C} & -0.3729440 & -4.9039000 & -3.6919680 \\ \mathrm{H} & 0.7343030 & -5.4214550 & -1.9079460 \\ \mathrm{H} & 1.1814110 & -3.8535720 & -2.5998210 \\ \mathrm{C} & -2.5506210 & -5.1338390 & -2.4444530 \\ \mathrm{H} & -1.4888490 & -5.6616360 & -0.6365120 \\ \mathrm{H} & -2.5485930 & -4.2513030 & -0.4592440 \\ \mathrm{C} & -1.5808820 & -5.8034420 & -3.4216360 \\ \mathrm{H} & 0.3383250 & -5.3959210 & -4.3643280 \\ \mathrm{H} & -0.7045440 & -3.9871080 & -4.1963210 \\ \mathrm{H} & -3.3980910 & -5.7936560 & -2.2286560 \\ \mathrm{H} & -2.9580520 & -4.2225480 & -2.9037490 \\ \mathrm{H} & -2.0939410 & -6.0416970 & -4.3589720 \\ \mathrm{H} & -1.2375510 & -6.7553040 & -2.9935330 \\ \mathrm{O} & -4.6668290 & 7.3731660 & -2.0584020 \\ \mathrm{O} & -8.0776120 & -3.0007930 & 1.5126960 \\ \mathrm{C} & -5.9382660 & 6.9079390 & -2.4579180 \\ \mathrm{H} & -6.4776230 & 6.4520100 & -1.6191490 \\ \mathrm{H} & -6.4857240 & 7.7829090 & -2.8080950 \\ \mathrm{H} & -5.8574010 & 6.1780900 & -3.2720520 \\ \mathrm{C} & -8.6184190 & -2.2275380 & 2.5631300 \\ \mathrm{H} & -9.5786740 & -2.6796110 & 2.8099360 \\ \mathrm{H} & -7.9693360 & -2.2465410 & 3.4468620 \\ \mathrm{H} & -8.7738200 & -1.1876650 & 2.2522990\end{array}$




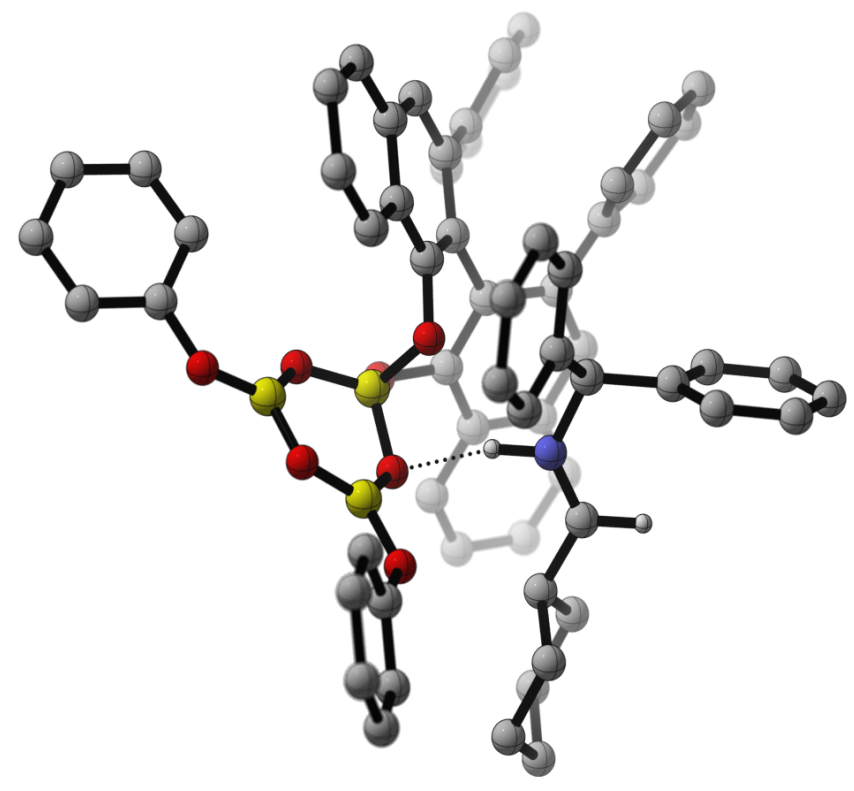

Boroxinate-H-Iminium Complex $\mathbf{4 g}$

File Name: catim4g

RRHO Thermochemistry $(T=298)$

Zero-point correction $(\mathrm{RRHO})=$

Thermal correction to Energy $(\mathrm{RRHO})=$

Thermal correction to Enthalpy $(\mathrm{RRHO})=$

1.055343 (Hartree/Particle)

1.115177

1.116121

Thermal correction to Gibbs Free Energy $(\mathrm{RRHO})=\quad 0.958917$

Sum of electronic and zero-point Energies $(\mathrm{RRHO})=\quad-3125.476145$

Sum of electronic and thermal Energies $(\mathrm{RRHO})=\quad-3125.416311$

Sum of electronic and thermal Enthalpies (RRHO) $=\quad-3125.415367$

Sum of electronic and thermal Free Energies $(\mathrm{RRHO})=\quad-3125.572571$

Quasi-RRHO Thermochemistry $(T=298, v 0=100 \mathrm{~cm}-1)$

Zero-point correction (QRRHO)=

Thermal correction to Energy $(\mathrm{QRRHO})=$

1.055343 (Hartree/Particle)

Thermal correction to Enthalpy $(Q R R H O)=$

1.115177

1.116121

Thermal correction to Gibbs Free Energy (QRRHO)=

Sum of electronic and zero-point Energies (QRRHO)=

0.973475

Sum of electronic and thermal Energies $(Q R R H O)=$

Sum of electronic and thermal Enthalpies $(Q R R H O)=$ $-3125.476145$

$-3125.416311$

Sum of electronic and thermal Free Energies $(\mathrm{QRRHO})=$ 
Quasi-harmonic Thermochemistry $(T=298$, cutoff $=100 \mathrm{~cm}-1)$

Zero-point correction $(\mathrm{QHO})=$

Thermal correction to Energy $(\mathrm{QHO})=$

Thermal correction to Enthalpy $(\mathrm{QHO})=$

Thermal correction to Gibbs Free Energy $(\mathrm{QHO})=$

Sum of electronic and zero-point Energies $(\mathrm{QHO})=$

Sum of electronic and thermal Energies $(\mathrm{QHO})=$

Sum of electronic and thermal Enthalpies $(\mathrm{QHO})=$

Sum of electronic and thermal Free Energies $(\mathrm{QHO})=$
055343 (Hartree/Particle)

1.115476

1.116121

0.975490

$-3125.476145$

$-3125.416012$

$-3125.415367$

$-3125.555998$

$\begin{array}{lrrr}\text { C } & 3.6868120 & -0.9045980 & 1.6846450 \\ \text { C } & 3.9695290 & -1.4368290 & 0.4203850 \\ \text { C } & 5.2451500 & -1.9677410 & 0.1933800 \\ \text { C } & 6.2081850 & -1.9678750 & 1.1967250 \\ \text { C } & 5.9134710 & -1.4375900 & 2.4502940 \\ \text { C } & 4.6478940 & -0.9062400 & 2.6902870 \\ \text { C } & 2.9519180 & -1.4854190 & -0.6674710 \\ \text { C } & 2.0895600 & -0.3769130 & -0.9746740 \\ \text { C } & 1.1281500 & -0.5303360 & -1.9697360 \\ \text { C } & 1.0157100 & -1.7441370 & -2.7178370 \\ \text { C } & 1.9022200 & -2.8136170 & -2.4323510 \\ \text { C } & 2.8427360 & -2.6589380 & -1.3841560 \\ \text { C } & 1.8147850 & -4.0006440 & -3.2086080 \\ \text { C } & 0.9018570 & -4.1041720 & -4.2291010 \\ \text { C } & 0.0182820 & -3.0325600 & -4.5089380 \\ \text { C } & 0.0646330 & -1.8804480 & -3.7619260 \\ \text { H } & -0.6988580 & -3.1275170 & -5.3187540 \\ \text { C } & 2.1670560 & 0.9376210 & -0.2720160 \\ \text { C } & 0.9845580 & 1.4440250 & 0.2424780 \\ \text { C } & 0.9362170 & 2.6491780 & 0.9990540 \\ \text { C } & 2.1350400 & 3.3854080 & 1.1717690 \\ \text { C } & 3.3335780 & 2.8834410 & 0.6035320 \\ \text { C } & 3.3718450 & 1.7005790 & -0.1020490 \\ \text { C } & -0.2796310 & 3.1301570 & 1.5478040 \\ \text { C } & -0.3022160 & 4.3211750 & 2.2287590 \\ \text { C } & 0.8863670 & 5.0769160 & 2.3843260 \\ \text { C } & 2.0757940 & 4.6189380 & 1.8739790 \\ \text { H } & -1.1773700 & 2.5281050 & 1.4303840 \\ \text { O } & -0.1816760 & 0.7940140 & 0.0240850 \\ \text { B } & -0.6944840 & 0.9207470 & -1.3677870 \\ \text { O } & 0.3092230 & 0.4810170 & -2.3188790 \\ \text { C } & 4.6653060 & 1.2785800 & -0.7024090 \\ \text { C } & 5.8406140 & 1.3518610 & 0.0538440 \\ \text { C } & 7.0615110 & 0.9660370 & -0.4891340 \\ \text { C } & 7.1268970 & 0.5000120 & -1.8000880 \\ \text { C } & 5.9642050 & 0.4340160 & -2.5661570 \\ \text { C } & 4.7430430 & 0.8224700 & -2.0237760 \\ \text { O } & -1.8700730 & 0.0146480 & -1.3528300 \\ \text { B } & -3.0331770 & 0.5349320 & -0.8867200\end{array}$

$\begin{array}{lrrr}\text { O } & -3.2516060 & 1.8847670 & -0.7936300 \\ \text { B } & -2.2512770 & 2.7520680 & -1.2366310 \\ \text { O } & -2.6056360 & 4.0775000 & -1.2011930 \\ \text { C } & -1.7987150 & 5.1480920 & -1.4901600 \\ \text { C } & -2.4447000 & 6.2978820 & -1.9438170 \\ \text { C } & -1.7097540 & 7.4483750 & -2.2090400 \\ \text { C } & -0.3293070 & 7.4593440 & -2.0193420 \\ \mathrm{C} & 0.3058430 & 6.3067170 & -1.5634380 \\ \mathrm{C} & -0.4166350 & 5.1463560 & -1.2972850 \\ \mathrm{O} & -3.9945750 & -0.3827680 & -0.4975100 \\ \mathrm{C} & -5.1902790 & -0.0802900 & 0.1045180 \\ \mathrm{C} & -5.3169780 & 0.9424890 & 1.0449140 \\ \mathrm{C} & -6.5445560 & 1.1438510 & 1.6706940 \\ \mathrm{C} & -7.6391840 & 0.3361320 & 1.3704410 \\ \mathrm{C} & -7.5013470 & -0.6805580 & 0.4282830 \\ \mathrm{C} & -6.2806830 & -0.8912810 & -0.2058710 \\ \mathrm{O} & -1.0660790 & 2.2879350 & -1.6663170 \\ \mathrm{~N} & -1.0026680 & -1.8853880 & 0.5935910 \\ \mathrm{C} & -1.5213450 & -3.0193740 & 0.3113420 \\ \mathrm{C} & -0.1546230 & -1.5550590 & 1.7610980 \\ \mathrm{C} & 0.2359670 & -2.7983820 & 2.5352600 \\ \mathrm{C} & -0.5058010 & -3.2373630 & 3.6340300 \\ \mathrm{C} & -0.1414450 & -4.4046910 & 4.3008490 \\ \mathrm{C} & 0.9659890 & -5.1357570 & 3.8765100 \\ \mathrm{C} & 1.7085820 & -4.7008990 & 2.7804500 \\ \mathrm{C} & 1.3432660 & -3.5373700 & 2.1099710 \\ \mathrm{C} & -0.8224740 & -0.4862440 & 2.6067990 \\ \mathrm{C} & -0.0070910 & 0.2612050 & 3.4592530 \\ \mathrm{C} & -0.5582250 & 1.2190510 & 4.3014980 \\ \mathrm{C} & -1.9356310 & 1.4340620 & 4.3060180 \\ \mathrm{C} & -2.7522560 & 0.6914010 & 3.4593280 \\ \mathrm{C} & -2.1993900 & -0.2642480 & 2.6044670 \\ \mathrm{H} & -4.4669800 & 1.5773510 & 1.2655160 \\ \mathrm{H} & -6.6423210 & 1.9444740 & 2.3978670 \\ \mathrm{H} & -8.5918620 & 0.5004130 & 1.8630390 \\ \mathrm{H} & -8.3476710 & -1.3140280 & 0.1811150 \\ \mathrm{H} & -6.1575620 & -1.6744960 & -0.9476210 \\ \mathrm{H} & -3.5212230 & 6.2689160 & -2.0780310 \\ \mathrm{H} & -2.2202400 & 8.3388480 & -2.5635580\end{array}$




$\begin{array}{rrrr}\mathrm{H} & 0.2448580 & 8.3573290 & -2.2242330 \\ \mathrm{H} & 1.3805800 & 6.3006090 & -1.4069070 \\ \mathrm{H} & 0.0832430 & 4.2511500 & -0.9498950 \\ \mathrm{H} & 0.8522100 & 6.0249660 & 2.9124050 \\ \mathrm{H} & 2.9893250 & 5.1953360 & 1.9958460 \\ \mathrm{H} & 4.2396490 & 3.4773730 & 0.6927300 \\ \mathrm{H} & 5.7831210 & 1.6742450 & 1.0899430 \\ \mathrm{H} & 7.9601450 & 1.0132970 & 0.1190280 \\ \mathrm{H} & 8.0780190 & 0.1924860 & -2.2243190 \\ \mathrm{H} & 6.0079500 & 0.0848810 & -3.5934890 \\ \mathrm{H} & 3.8415300 & 0.7788910 & -2.6278130 \\ \mathrm{H} & 5.4930190 & -2.3409150 & -0.7961980 \\ \mathrm{H} & 7.1971220 & -2.3671270 & 0.9919900 \\ \mathrm{H} & 6.6653140 & -1.4325210 & 3.2335310 \\ \mathrm{H} & 4.4062890 & -0.4905270 & 3.6640200 \\ \mathrm{H} & 2.7122800 & -0.4656930 & 1.8771470 \\ \mathrm{H} & 3.4871720 & -3.4984740 & -1.1348820 \\ \mathrm{H} & 2.4951790 & -4.8193240 & -2.9883550 \\ \mathrm{H} & 0.8532630 & -5.0099740 & -4.8261770 \\ \mathrm{H} & 0.7419610 & -1.1206000 & 1.3042700 \\ \mathrm{H} & -1.1826220 & -1.0933870 & -0.0550970 \\ \mathrm{H} & -1.3300090 & -3.8432750 & 1.0004810 \\ \mathrm{H} & -1.3593020 & -2.6575540 & 3.9744300 \\ \mathrm{H} & 1.9185430 & -3.1954780 & 1.2521050 \\ \mathrm{H} & -2.8531230 & -0.8277940 & 1.9414540 \\ \mathrm{H} & 1.0666770 & 0.0876000 & 3.4632810\end{array}$

$\begin{array}{rrrr}\mathrm{H} & -1.2336080 & 4.6913360 & 2.6460160 \\ \mathrm{H} & -0.6025500 & -1.0486590 & -3.9598360 \\ \mathrm{H} & 0.0885100 & 1.8054590 & 4.9462750 \\ \mathrm{H} & -2.3686310 & 2.1806260 & 4.9641850 \\ \mathrm{H} & -3.8269110 & 0.8457340 & 3.4601460 \\ \mathrm{H} & 2.5769720 & -5.2621730 & 2.4508430 \\ \mathrm{H} & 1.2532030 & -6.0406460 & 4.4028130 \\ \mathrm{H} & -0.7190350 & -4.7380770 & 5.1570960 \\ \mathrm{C} & -2.3488260 & -3.2564560 & -0.8940500 \\ \mathrm{C} & -1.7032550 & -4.3794030 & -1.7292870 \\ \mathrm{C} & -3.7754880 & -3.6536480 & -0.4633820 \\ \mathrm{H} & -2.3910760 & -2.3344000 & -1.4863690 \\ \mathrm{C} & -2.5637390 & -4.6756080 & -2.9580000 \\ \mathrm{H} & -1.6190820 & -5.2846710 & -1.1106350 \\ \mathrm{H} & -0.6917760 & -4.0875260 & -2.0289360 \\ \mathrm{C} & -4.6244840 & -3.9500400 & -1.7010100 \\ \mathrm{H} & -3.7261120 & -4.5492820 & 0.1721710 \\ \mathrm{H} & -4.2232750 & -2.8479090 & 0.1272640 \\ \mathrm{C} & -3.9936350 & -5.0485920 & -2.5605410 \\ \mathrm{H} & -2.0995180 & -5.4762130 & -3.5441220 \\ \mathrm{H} & -2.5821530 & -3.7835430 & -3.5973240 \\ \mathrm{H} & -5.6362450 & -4.2347530 & -1.3922330 \\ \mathrm{H} & -4.7135930 & -3.0297360 & -2.2946540 \\ \mathrm{H} & -4.6018080 & -5.2249390 & -3.4536290 \\ \mathrm{H} & -3.9801500 & -5.9884490 & -1.9915930 \\ & & & \end{array}$




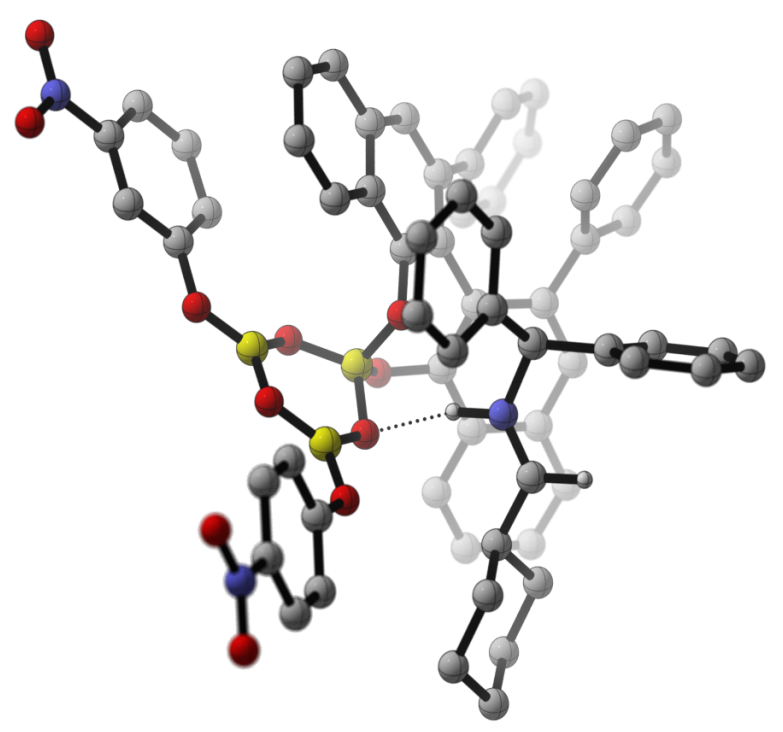

Boroxoinate-H-Iminium Complex 4h

File Name: catim4h

RRHO Thermochemistry $(T=298)$

Zero-point correction $(\mathrm{RRHO})=$

1.060936 (Hartree/Particle)

Thermal correction to Energy $(\mathrm{RRHO})=$

1.126019

Thermal correction to Enthalpy $(\mathrm{RRHO})=$

1.126962

Thermal correction to Gibbs Free Energy $(\mathrm{RRHO})=$

Sum of electronic and zero-point Energies $(\mathrm{RRHO})=$

0.955587

Sum of electronic and thermal Energies $(\mathrm{RRHO})=$

$-3534.333384$

Sum of electronic and thermal Enthalpies $(\mathrm{RRHO})=$

$-3534.268301$

Sum of electronic and thermal Free Energies $(\mathrm{RRHO})=$

$-3534.267358$

$-3534.438733$

Quasi-RRHO Thermochemistry $(T=298, v 0=100 \mathrm{~cm}-1)$

Zero-point correction (QRRHO)=

1.060936 (Hartree/Particle)

Thermal correction to Energy $(\mathrm{QRRHO})=$

1.126019

Thermal correction to Enthalpy (QRRHO)=

1.126962

Thermal correction to Gibbs Free Energy $(\mathrm{QRRHO})=$

0.973137

Sum of electronic and zero-point Energies $(Q R R H O)=$

$-3534.333384$

Sum of electronic and thermal Energies $(Q R R H O)=$ $-3534.268301$

Sum of electronic and thermal Enthalpies (QRRHO)= $-3534.267358$

Sum of electronic and thermal Free Energies (QRRHO)=

$-3534.421183$

Quasi-harmonic Thermochemistry $(T=298$, cutoff $=100 \mathrm{~cm}-1)$

Zero-point correction $(\mathrm{QHO})=$

Thermal correction to Energy $(\mathrm{QHO})=$

Thermal correction to Enthalpy $(\mathrm{QHO})=$
1.060936 (Hartree/Particle)

1.126362

1.126962 
Thermal correction to Gibbs Free Energy (QHO)=

Sum of electronic and zero-point Energies $(\mathrm{QHO})=$ Sum of electronic and thermal Energies $(\mathrm{QHO})=$ Sum of electronic and thermal Enthalpies $(\mathrm{QHO})=$ Sum of electronic and thermal Free Energies (QHO)=

$\begin{array}{lrrr}\text { C } & 4.1170950 & -0.1052960 & 1.9629110 \\ \text { C } & 4.6445070 & -0.6451520 & 0.7831670 \\ \text { C } & 6.0276810 & -0.8489320 & 0.7053710 \\ \text { C } & 6.8588030 & -0.5240730 & 1.7719500 \\ \text { C } & 6.3216010 & 0.0105530 & 2.9403680 \\ \text { C } & 4.9467920 & 0.2179140 & 3.0318080 \\ \text { C } & 3.7830350 & -1.0345340 & -0.3688960 \\ \text { C } & 2.7140650 & -0.2050510 & -0.8543340 \\ \text { C } & 1.9263580 & -0.6712290 & -1.9024310 \\ \text { C } & 2.1922280 & -1.9240720 & -2.5380030 \\ \text { C } & 3.2782620 & -2.7108580 & -2.0762740 \\ \text { C } & 4.0361370 & -2.2485560 & -0.9722030 \\ \text { C } & 3.5712440 & -3.9311340 & -2.7426740 \\ \text { C } & 2.8306500 & -4.3320600 & -3.8271780 \\ \text { C } & 1.7459160 & -3.5425950 & -4.2829680 \\ \text { C } & 1.4233670 & -2.3694620 & -3.6445310 \\ \text { H } & 1.1687370 & -3.8710380 & -5.1420460 \\ \text { C } & 2.4010520 & 1.1382230 & -0.2836820 \\ \text { C } & 1.0888900 & 1.3690480 & 0.0917080 \\ \text { C } & 0.6728450 & 2.5802460 & 0.7141000 \\ \text { C } & 1.6341230 & 3.6059680 & 0.8986870 \\ \text { C } & 2.9671920 & 3.3792340 & 0.4694790 \\ \text { C } & 3.3617130 & 2.1915860 & -0.1065320 \\ \text { C } & -0.6669120 & 2.7808880 & 1.1303210 \\ \text { C } & -1.0430270 & 3.9791070 & 1.6857380 \\ \text { C } & -0.0961840 & 5.0200320 & 1.8458780 \\ \text { C } & 1.2119010 & 4.8351770 & 1.4701190 \\ \text { H } & -1.3758220 & 1.9631410 & 1.0209160 \\ \text { O } & 0.1434740 & 0.4279640 & -0.1530070 \\ \text { B } & -0.2347880 & 0.3353240 & -1.5848640 \\ \text { O } & 0.9224490 & 0.0724890 & -2.4128740 \\ \text { C } & 4.7701310 & 2.0605490 & -0.5651820 \\ \text { C } & 5.8143800 & 2.4888610 & 0.2622060 \\ \text { C } & 7.1384850 & 2.3787400 & -0.1489000 \\ \text { C } & 7.4400700 & 1.8381800 & -1.3966890 \\ \text { C } & 6.4071200 & 1.4188200 & -2.2334090 \\ \text { C } & 5.0822710 & 1.5309900 & -1.8231110 \\ \text { O } & -1.1912520 & -0.8016960 & -1.5955060 \\ \text { B } & -2.4800300 & -0.5192220 & -1.3036360 \\ \text { O } & -2.9861960 & 0.7550150 & -1.3023240 \\ \text { C } & -2.1339980 & 1.8019180 & -1.6544250 \\ \text { C } & -2.7159940 & 3.0480330 & -1.5830990 \\ & -2.0828740 & 4.2553010 & -1.5356200 \\ & -2.3023990 & 6.5829580 & -1.0513410\end{array}$

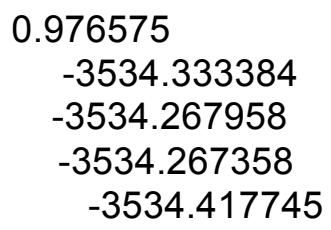

$\begin{array}{lrrr}\mathrm{C} & -0.9546370 & 6.8147430 & -1.2874870 \\ \mathrm{C} & -0.1752700 & 5.7265480 & -1.6633180 \\ \mathrm{C} & -0.7220130 & 4.4530230 & -1.7970170 \\ \mathrm{O} & -3.2849260 & -1.6093270 & -0.9901320 \\ \mathrm{C} & -4.5613250 & -1.5687200 & -0.5241100 \\ \mathrm{C} & -5.0404960 & -0.5369650 & 0.2911800 \\ \mathrm{C} & -6.3343030 & -0.6045990 & 0.7880930 \\ \mathrm{C} & -7.1266260 & -1.6999690 & 0.4665010 \\ \mathrm{C} & -6.6676040 & -2.7318610 & -0.3438800 \\ \mathrm{C} & -5.3756150 & -2.6611240 & -0.8394370 \\ \mathrm{O} & -0.8658810 & 1.5684240 & -2.0227110 \\ \mathrm{~N} & -0.0481190 & -2.3249200 & 0.5670220 \\ \mathrm{C} & -0.2267190 & -3.5670090 & 0.3221420 \\ \mathrm{C} & 0.5419910 & -1.7229370 & 1.7839310 \\ \mathrm{C} & 1.1238610 & -2.7794080 & 2.7007330 \\ \mathrm{C} & 0.3744200 & -3.3353450 & 3.7396430 \\ \mathrm{C} & 0.9248740 & -4.3328120 & 4.5406520 \\ \mathrm{C} & 2.2254270 & -4.7761490 & 4.3101890 \\ \mathrm{C} & 2.9763300 & -4.2234400 & 3.2745530 \\ \mathrm{C} & 2.4265760 & -3.2296350 & 2.4700220 \\ \mathrm{C} & -0.4737590 & -0.8117760 & 2.4492170 \\ \mathrm{C} & 0.0121590 & 0.1854460 & 3.2967850 \\ \mathrm{C} & -0.8651100 & 1.0222870 & 3.9760910 \\ \mathrm{C} & -2.2414180 & 0.8664240 & 3.8187930 \\ \mathrm{C} & -2.7309900 & -0.1267210 & 2.9761000 \\ \mathrm{C} & -1.8512070 & -0.9633530 & 2.2867640 \\ \mathrm{H} & -4.4072400 & 0.3107480 & 0.5196010 \\ \mathrm{H} & -6.7335580 & 0.1797580 & 1.4195430 \\ \mathrm{H} & -7.3181590 & -3.5661840 & -0.5746580 \\ \mathrm{H} & -4.9785080 & -3.4423210 & -1.4788050 \\ \mathrm{H} & -3.9398220 & 5.1834640 & -0.9630140 \\ \mathrm{H} & -0.5449670 & 7.8102510 & -1.1773990 \\ \mathrm{H} & 0.8845400 & 5.8649310 & -1.8501820 \\ \mathrm{H} & -0.0996500 & 3.6142960 & -2.0793070 \\ \mathrm{H} & -0.4100110 & 5.9683990 & 2.2715710 \\ \mathrm{H} & 1.9419850 & 5.6303680 & 1.5981190 \\ \mathrm{H} & 3.6882340 & 4.1871850 & 0.5649100 \\ \mathrm{H} & 5.5821470 & 2.8704150 & 1.2527360 \\ \mathrm{H} & 7.9363340 & 2.7003560 & 0.5140400 \\ \mathrm{H} & 6.6322310 & 1.0082330 & -3.2131680 \\ \mathrm{H} & 6.4550600 & -1.2220690 & -0.2209750 \\ \mathrm{H} & -1.9691770 & 0.2692620 & 3.7724740\end{array}$




$\begin{array}{rrrrrrrr}\mathrm{H} & 4.5167440 & 0.6332480 & 3.9382920 & \mathrm{C} & -2.2111560 & -4.7065740 & -0.5853440 \\ \mathrm{H} & 3.0500500 & 0.0829960 & 2.0390070 & \mathrm{H} & -1.0123540 & -3.2154300 & -1.6060070 \\ \mathrm{H} & 4.8364500 & -2.8775470 & -0.5901060 & \mathrm{C} & -0.5713530 & -5.6442940 & -2.8587740 \\ \mathrm{H} & 4.4045790 & -4.5321300 & -2.3878670 & \mathrm{H} & 0.2721890 & -5.9123550 & -0.8855940 \\ \mathrm{H} & 3.0736160 & -5.2578580 & -4.3400520 & \mathrm{H} & 1.0452050 & -4.6266020 & -1.8265060 \\ \mathrm{H} & 1.3545710 & -1.1016680 & 1.3901380 & \mathrm{C} & -2.8535740 & -5.2623660 & -1.8578310 \\ \mathrm{H} & -0.3528300 & -1.6393590 & -0.1481930 & \mathrm{H} & -2.0558800 & -5.5209140 & 0.1359810 \\ \mathrm{H} & 0.0924700 & -4.2793560 & 1.0842090 & \mathrm{H} & -2.8652200 & -3.9644880 & -0.1152930 \\ \mathrm{H} & -0.6335320 & -2.9769180 & 3.9295550 & \mathrm{C} & -1.9344940 & -6.2692850 & -2.5544340 \\ \mathrm{H} & 3.0082300 & -2.7964030 & 1.6587560 & \mathrm{H} & 0.0972770 & -6.3751880 & -3.3260830 \\ \mathrm{H} & -2.2521890 & -1.7337110 & 1.6303800 & \mathrm{H} & -0.6967130 & -4.8235390 & -3.5767640 \\ \mathrm{H} & 1.0856930 & 0.3044000 & 3.4247020 & \mathrm{H} & -3.8142460 & -5.7270730 & -1.6100610 \\ \mathrm{H} & -2.0708740 & 4.1336220 & 2.0005750 & \mathrm{H} & -3.0623620 & -4.4266180 & -2.5401920 \\ \mathrm{H} & 0.5975670 & -1.7505660 & -3.9785580 & \mathrm{H} & -2.4014830 & -6.6294970 & -3.4766110 \\ \mathrm{H} & -0.4740650 & 1.8037560 & 4.6195810 & \mathrm{H} & -1.7974980 & -7.1427910 & -1.9024460 \\ \mathrm{H} & -2.9282010 & 1.5180210 & 4.3494080 & \mathrm{~N} & -3.1527650 & 7.7108950 & -0.6482180 \\ \mathrm{H} & -3.8015350 & -0.2627950 & 2.8567210 & \mathrm{~N} & -8.4869860 & -1.7715390 & 1.0001000 \\ \mathrm{H} & 3.9927360 & -4.5594740 & 3.0965420 & \mathrm{O} & -4.3330080 & 7.4907200 & -0.4391080 \\ \mathrm{H} & 2.6554200 & -5.5478570 & 4.9408360 & \mathrm{O} & -2.6301720 & 8.8070670 & -0.5422540 \\ \mathrm{H} & 0.3397530 & -4.7573210 & 5.3501240 & \mathrm{O} & -8.8671670 & -0.8593430 & 1.7136180 \\ \mathrm{C} & -0.8502810 & -4.0622120 & -0.9270420 & \mathrm{O} & -9.1634560 & -2.7418010 & 0.7039770 \\ \mathrm{C} & 0.0823890 & -5.0912980 & -1.5921490 & & & & \end{array}$

\section{Electrostatic Potential Slice Maps:}

Optimizations of catalyst-borox-iminium complexes were carried out at the $M 06-2 X / 6-31 G^{*}$ level of theory as implemented by the Gaussian 16 software package with bulk solvent effects accounted for with the self-consistent reaction field polarizable continuum model (PCM) as implemented for toluene. Electrostatic slice maps were generated from NPA charges calculated from these optimized structures using the cubegen function provided by Gaussian 16. These were plotted in Jmol to create the colored-in field representing charge such that the outer edges of the map would have a neutral (green) charge using the commands below. The coordinates in brackets following "isosurface plane" represent the plane containing the (im) $\mathrm{N}-\mathrm{H}-\mathrm{O}(\mathrm{cat})$ interaction between the catalyst and the substrate. The coloring gradient was kept consistent between derivatives for accurate qualitative comparison.

No2 derivative:

isosurface plane $\{0.53651-1.531771 .48689\}\left\{\begin{array}{lll}-0.01663-1.26501 & 0.65245\}\{-1.20492-0.69855\end{array}\right.$ $-0.75211\}$ color absolute $-0.160 .08 \mathrm{map} " \mathrm{C}: /<$ full path to cube file>/M06catnitimphen_ESP_potential.cube" translucent; show isosurface 
Ph derivative:

isosurface plane $\{-0.71944-2.040820 .79600\}\left\{\begin{array}{llll}-1.62233 & 0.10086 & -0.64239\end{array}\{-1.02183-1.29391\right.$

$0.11814\}$ color absolute -0.160 .08 map $\mathrm{C}: /<$ full path to cube file>/M06cathexSP-

NPA_redo_Pot.cube" translucent; show isosurface

\section{Quantitative Electrostatic Potential Data:}

Quantitative ESP data was collected by feeding G16 a grid (using the prop=grid keyword) in a fort.52 file that consisted of the coordinates of the substrate, while the catalyst structure was kept in the main input file. The following line was included after the input coordinates to trap the calculated ESP data for the complex in a fort.99 file: "39 252 99", where 39 is the atom count in the fort.52 file, 52 is the designation of the input file (i.e. telling G16 that fort.52 is the input for the grid), and 99 designates the output file for ESP data. An example input file and job file are included below, as well as the quantitative ESP data spreadsheet. Charges on the substrate were calculated with the pop=NPA command in a separate single-point calculation.

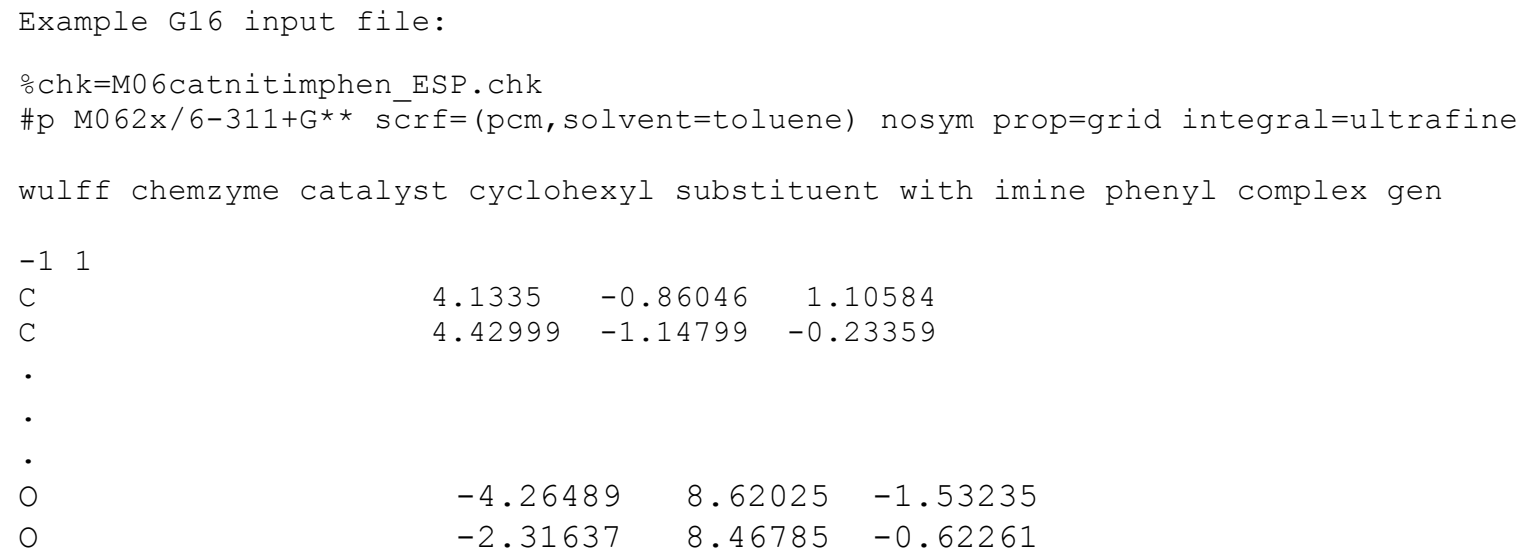


module add Gaussian

export OMP NUM THREADS $=16$

export GAUSSS_S $\bar{C} R D I R=/$ data/home/\$USER/scratch/\$SLURM_JOBID

mkdir -p \$GAUSS SCRDIR

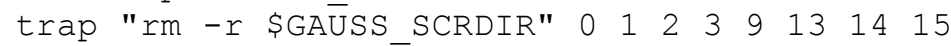

df $-1 h$

- /etc/profile.d/modules.sh

find \$SLURM_SUBMIT_DIR -maxdepth 1 -name "fort.*" -exec cp \{\} . ;

cP \$SLURM_SÜBMIT_DIR/M06catnitimphen_ESP.chk .

/cm/shared/apps/Gaussian/g09/g09 -m="28GB" -p=16

\$SLURM_SUBMIT_DIR/M06catnitimphen_ESP.com \$SLURM_SUBMIT_DIR/M06catnitimphen_ESP.log rm -f Gau* - \#Clean up any temporary files from crashed job

/cm/shared/apps/Gaussian/g09/formchk M06catnitimphen_ESP.chk

cP M06catnitimphen_ESP.chk \$SLURM_SUBMIT_DIR/.

cp M06catnitimphen_ESP.fchk \$SLURM_SUBMIT_DIR/.

find. -maxdepth 1 -name "fort.*" -exec cp \{\} \$SLURM_SUBMIT_DIR/.

exit

Example fort.52 input file (just coordinates-no atom names):
$0.53651-1.53177$
$1.3468-2.53002$
1.48689
1.43379

$3.01265-2.24314$

7.02462

$0.94163-3.42951$

6.34637

\section{Quantitative ESP data:}

$\begin{array}{lcccc}\text { fort.99 } & \begin{array}{c}\text { NPA charge } \\ \text { substrate }\end{array} & \text { Stab. Energy } \mathrm{R}^{3}=4-\mathrm{NO}_{2} \mathrm{Ph}(\mathrm{kcal} / \mathrm{mol}) & & \text { Stab. Energy } \mathrm{R}^{3}=\mathrm{Cy} \\ -0.12427 & -0.46035 & 35.9 & <-\mathrm{N}--> \\ -0.10437 & 0.28686 & -18.8 & -23.1 \\ -0.1039 & -0.17778 & 11.6 & 3.2 \\ -0.08787 & -0.13045 & 7.2 & 5.5 \\ -0.07694 & -0.20313 & 9.8 & 9.5 \\ -0.08054 & -0.13634 & 6.9 & 7.8 \\ -0.09661 & -0.19766 & 12.0 & 7.4 \\ -0.11344 & -0.15214 & 10.8 & 8.8 \\ -0.1071 & -0.04099 & 2.8 & 10.9 \\ -0.08079 & -0.09644 & 4.9 & \\ -0.0706 & -0.20613 & 9.1 & \\ -0.06036 & -0.19401 & 7.3 & \\ -0.05708 & -0.18829 & 6.7 & \\ -0.06063 & -0.1961 & 7.5 & \\ -0.07271 & -0.19687 & 9.0 & \\ -0.09621 & -0.06594 & 4.0 & \\ -0.08216 & -0.19566 & 10.1 & \mathrm{H}(\mathrm{Cy})--> & 9.4 \\ & & & \\ \end{array}$




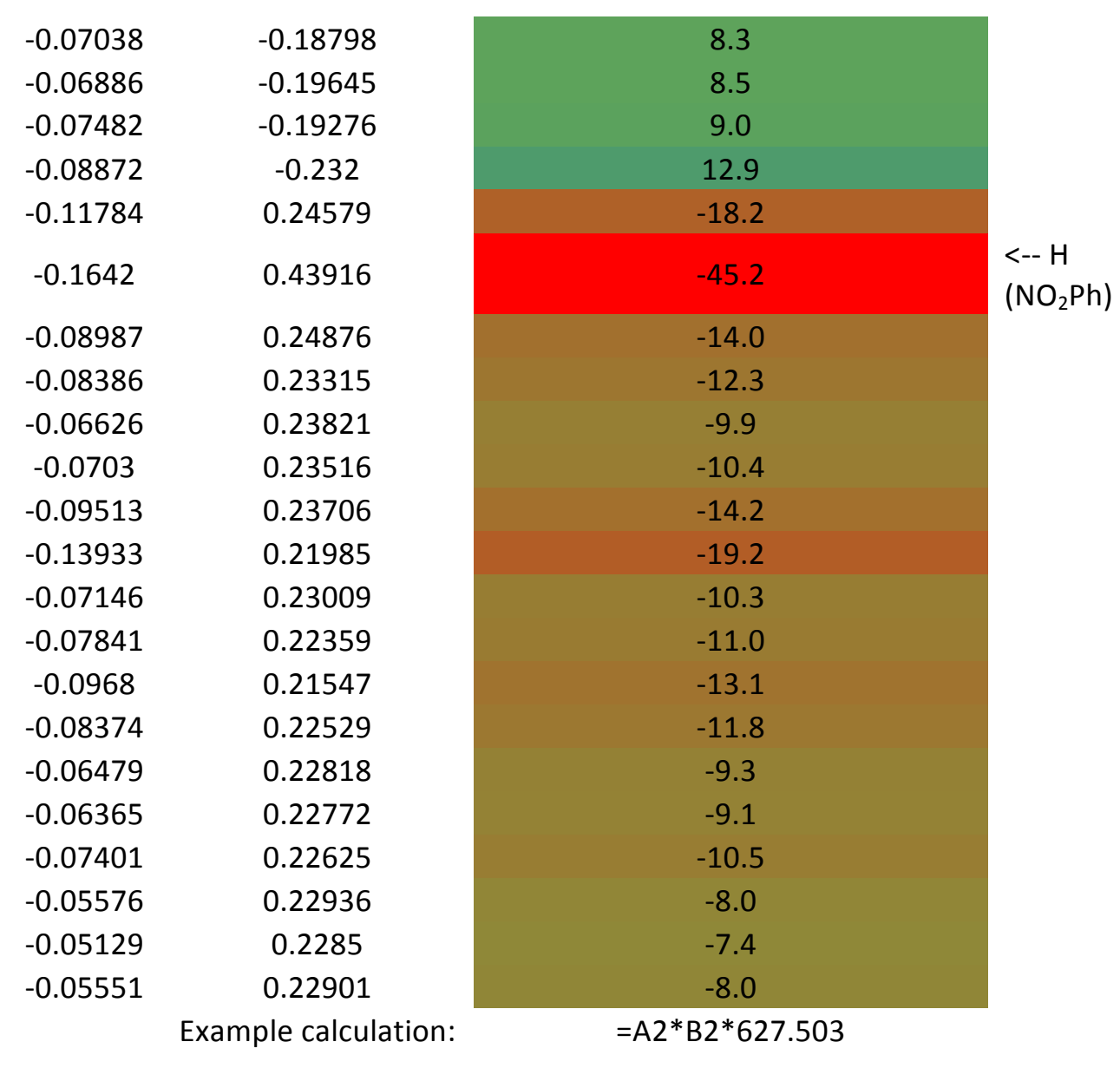

\begin{tabular}{|c|}
\hline-14.0 \\
\hline-10.8 \\
\hline-13.8 \\
\hline-12.8 \\
\hline-11.8 \\
\hline-10.8 \\
\hline-9.5 \\
\hline-10.1 \\
\hline-9.8 \\
\hline-8.0 \\
\hline-8.4 \\
\hline 20.9 \\
\hline 22.8 \\
\hline 21.1 \\
\hline-19.0 \\
\hline 19.9 \\
\hline-10.8 \\
\hline-14.7 \\
\hline 17.9 \\
\hline-10.2 \\
\hline-12.8 \\
\hline 17.0 \\
\hline-10.0 \\
\hline-11.0 \\
\hline-8.5 \\
\hline-9.7 \\
\hline-8.4 \\
\hline-7.9 \\
\hline
\end{tabular}

$\begin{array}{cc}\text { Scaling factors for Jmol } & \text { Scaling factors }(\mathrm{kcal} / \mathrm{mol}) \\ -0.16 & -100.40048 \\ 0.08 & 50.20024 \\ \text { Example calculation: } & =\mathrm{L} 12 * 627.503\end{array}$

Difference in $\mathrm{H}$-bond Stab. Energy =

$$
\begin{gathered}
(-46.0 \mathrm{kcal} / \mathrm{mol})-(-45.2 \mathrm{kcal} / \mathrm{mol}) \\
-\quad \text { - } 8 \mathrm{kral} / \mathrm{mnl})
\end{gathered}
$$

Catalyst 5,5' substitution analysis: 


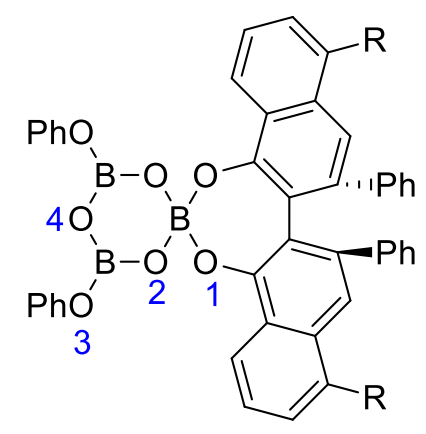

Calculated ADCH charges on numbered oxygens

\begin{tabular}{cccccc}
\hline Entry & $\mathbf{0 1}$ & $\mathbf{O 2}$ & $\mathbf{0 3}$ & $\mathbf{0 4}$ & Substitution \\
\hline 1 & -0.22315 & -0.27277 & -0.18776 & -0.24852 & $\mathrm{H}$ \\
2 & -0.33572 & -0.34204 & -0.30230 & -0.24970 & $\mathrm{Me}$ \\
3 & -0.33412 & -0.34185 & -0.30119 & -0.24637 & $\mathrm{OMe}$ \\
4 & -0.32791 & -0.34049 & -0.30028 & -0.34049 & $\mathrm{Br}$ \\
5 & -0.32980 & -0.33779 & -0.29442 & -0.25586 & $\mathrm{Cl}$ \\
6 & -0.32538 & -0.34079 & -0.30106 & -0.26235 & $\mathrm{CF} 3$ \\
\hline
\end{tabular}

Difference in $\mathrm{ADCH}$ charge with respect to $\mathrm{R}=\mathrm{H}$

\begin{tabular}{cccccc}
\hline Entry & $\mathbf{O 1}$ & $\mathbf{0 2}$ & $\mathbf{0 3}$ & $\mathbf{0 4}$ & Substitution \\
\hline 1 & 0.00000 & 0.00000 & 0.00000 & 0.00000 & $\mathrm{H}$ \\
2 & -0.11257 & -0.06927 & -0.11455 & -0.00118 & $\mathrm{OMe}$ \\
3 & -0.11097 & -0.06908 & -0.11343 & 0.00214 & $\mathrm{Me}$ \\
4 & -0.10476 & -0.06772 & -0.11253 & -0.09197 & $\mathrm{Br}$ \\
5 & -0.10666 & -0.06502 & -0.10667 & -0.00734 & $\mathrm{Cl}$ \\
6 & -0.10224 & -0.06802 & -0.11330 & -0.01383 & $\mathrm{CF} 3$ \\
\hline
\end{tabular}

$\mathrm{ADCH}$ charges were calculated on the numbered oxygens in the BOROX ring on the catalyst with various functional group substitutions on the $5,5^{\prime}$ positions of the hydrocarbon backbone of the catalyst including methoxy $(\mathrm{OMe})$, methyl $(\mathrm{Me})$, bromo $(\mathrm{Br})$, chloro $(\mathrm{Cl})$, and trifluoromethyl $\left(\mathrm{CF}_{3}\right)$ substitutions. These were first optimized at the M06-2X/6-31G* level of theory with bulk solvent effects accounted for by the PCM model for toluene included in the Gaussian 16 package. ADCH charges were then calculated using multiwfn from the resulting checkpoint file. A slight decrease in negative charge is observed as the electron withdrawing nature of the substituting group increases for $\mathrm{O} 1$, but this trend is not observed for $\mathrm{O} 2, \mathrm{O} 3$, and $\mathrm{O} 4$. Since $\mathrm{O} 2$ is the oxygen that interacts with the substrate, this suggests that 5,5 ' substitution should not have a significant effect on the activity of the catalyst. 
IV. ${ }^{1} \mathrm{Hand}{ }^{13} \mathrm{C}$ spectra for compounds $22 \mathrm{a}, 22 \mathrm{~b}, 23 \mathrm{a}, 23 \mathrm{~b}$ and 41.<smiles>C(=N/C(c1ccccc1)c1ccccc1)\c1ccccc1</smiles>

22a

(E)-N-benzhydryl-1-phenylmethanimine 22a- ${ }^{1} \mathrm{H}$ NMR (500 $\mathrm{MHz}, \mathrm{CDCl}_{3}$ )

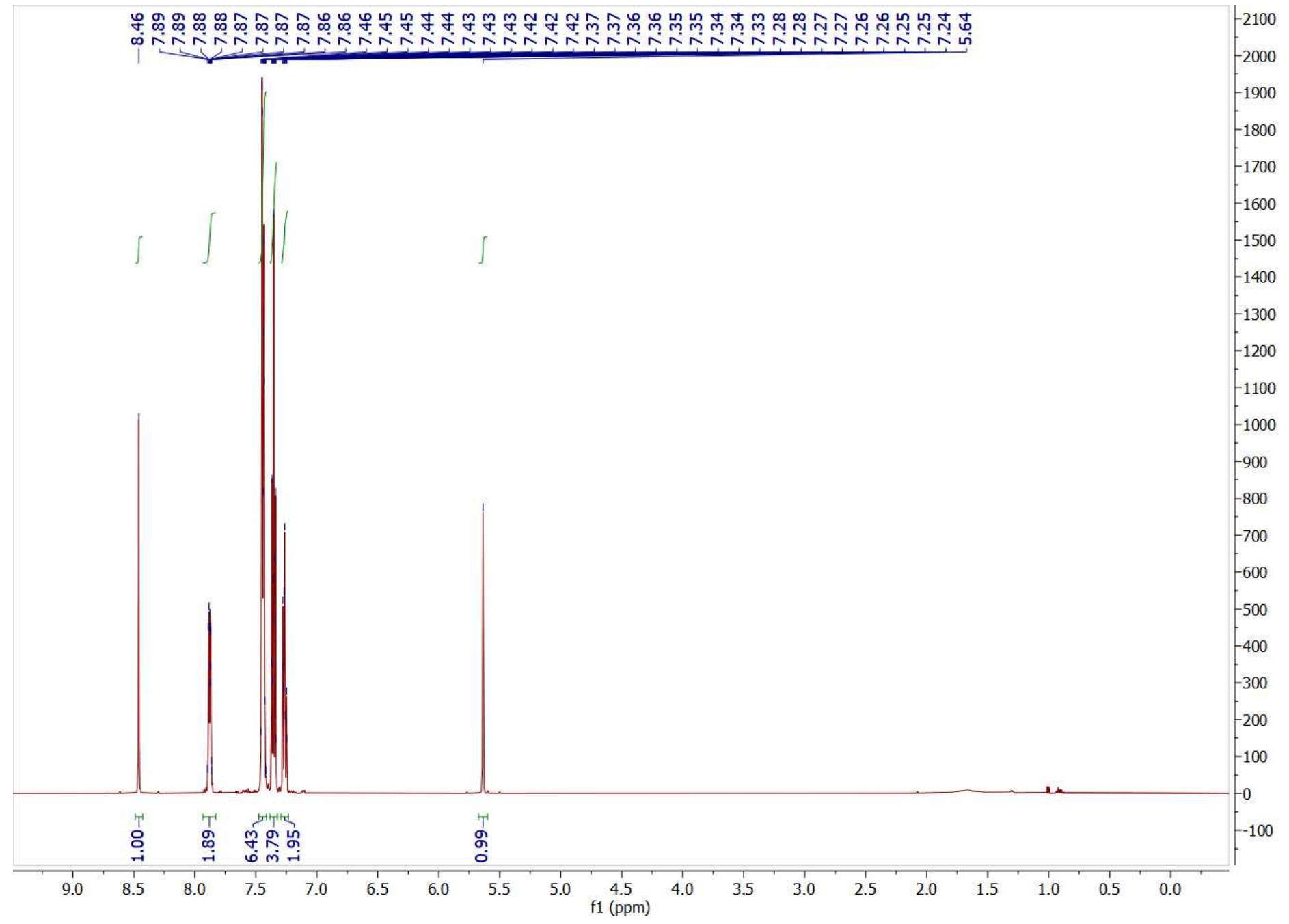




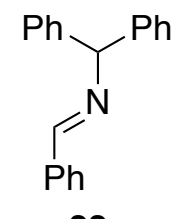

$22 a$

(E)-N-benzhydryl-1-phenylmethanimine $22 \mathbf{a}-{ }^{13} \mathrm{C}\left\{{ }^{1} \mathrm{H}\right\}\left(126 \mathrm{MHz}, \mathrm{CDCl}_{3}\right)$

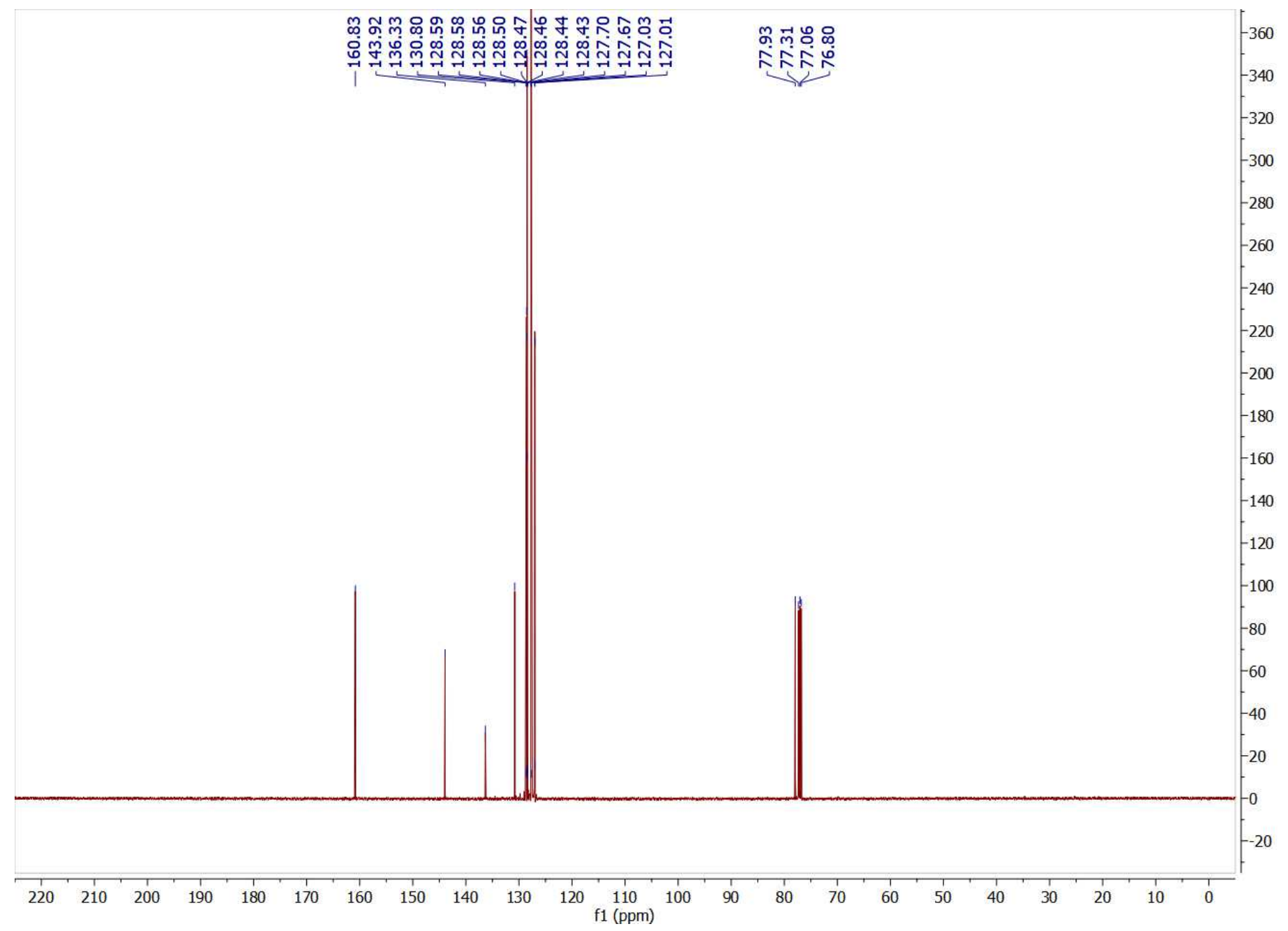




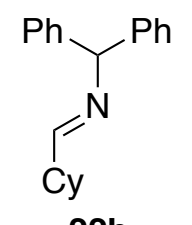

22b

(E)-N-benzhydryl-1-cyclohexylmethanimine 22b- ${ }^{1} \mathrm{H}$ NMR $\left(500 \mathrm{MHz}, \mathrm{CDCl}_{3}\right.$ )

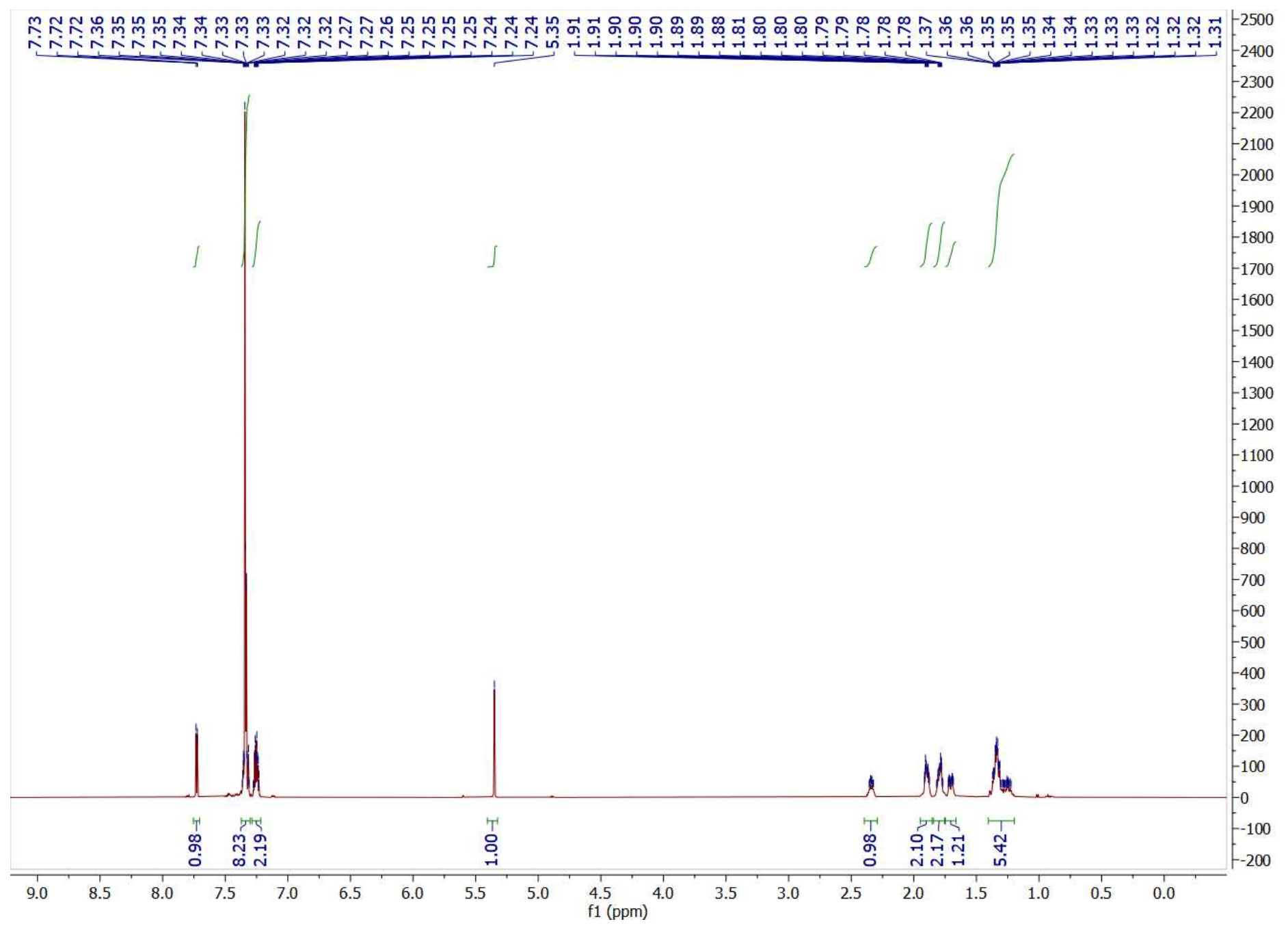


<smiles>[R60][CH]/C=N/C(c1ccccc1)c1ccccc1</smiles>

(E)-N-benzhydryl-1-cyclohexylmethanimine $22 \mathbf{b}-{ }^{13} \mathrm{C}\left\{{ }^{1} \mathrm{H}\right\}\left(126 \mathrm{MHz}, \mathrm{CDCl}_{3}\right)$

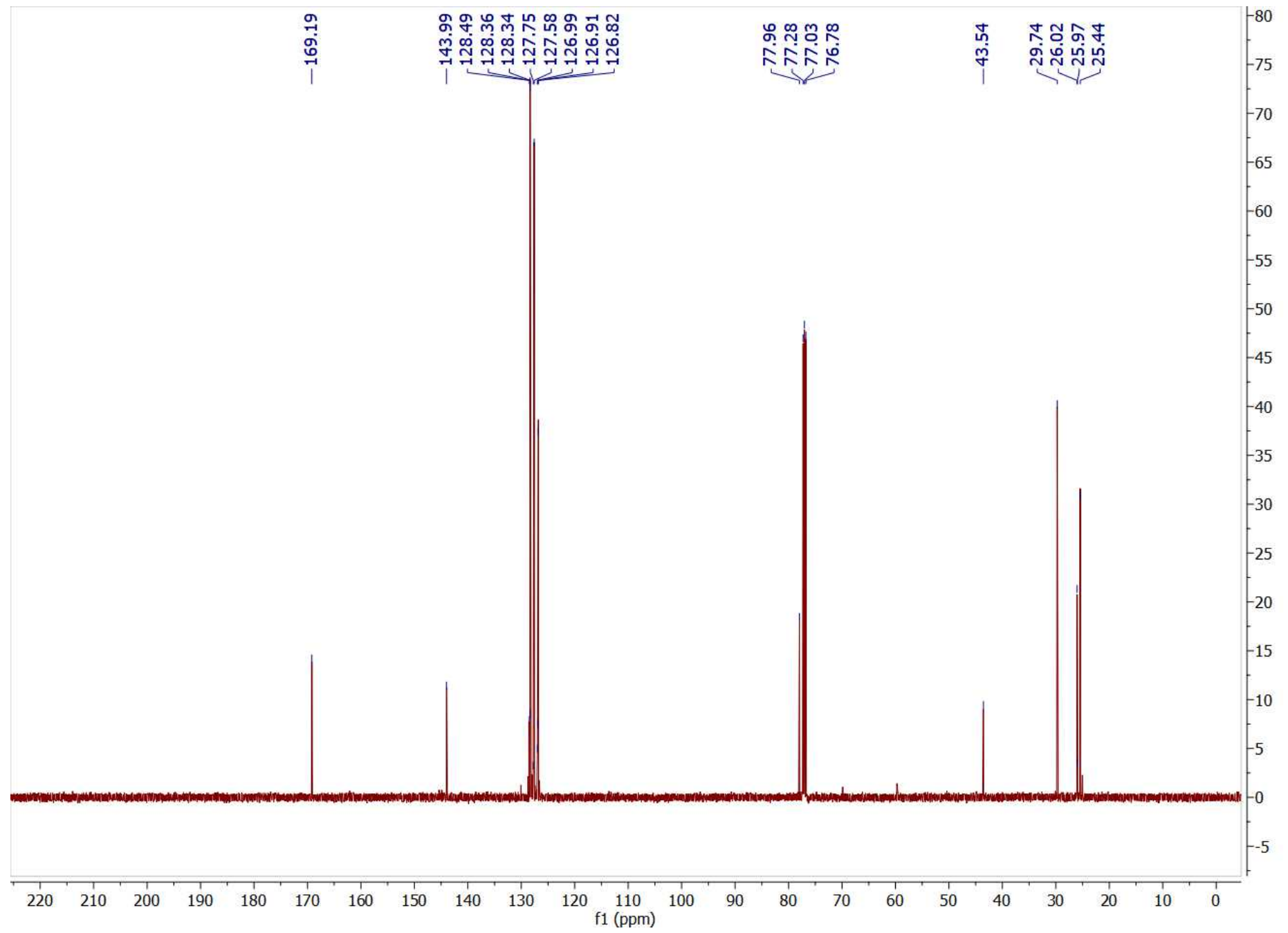




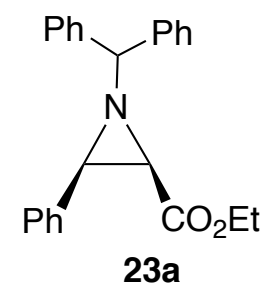

(2R,3R)-ethyl-1-benzhydryl-3-phenylaziridine-2-carboxylate $23 \mathbf{a}-{ }^{1} \mathrm{H}$ NMR $\left(500 \mathrm{MHz}, \mathrm{CDCl}_{3}\right)$

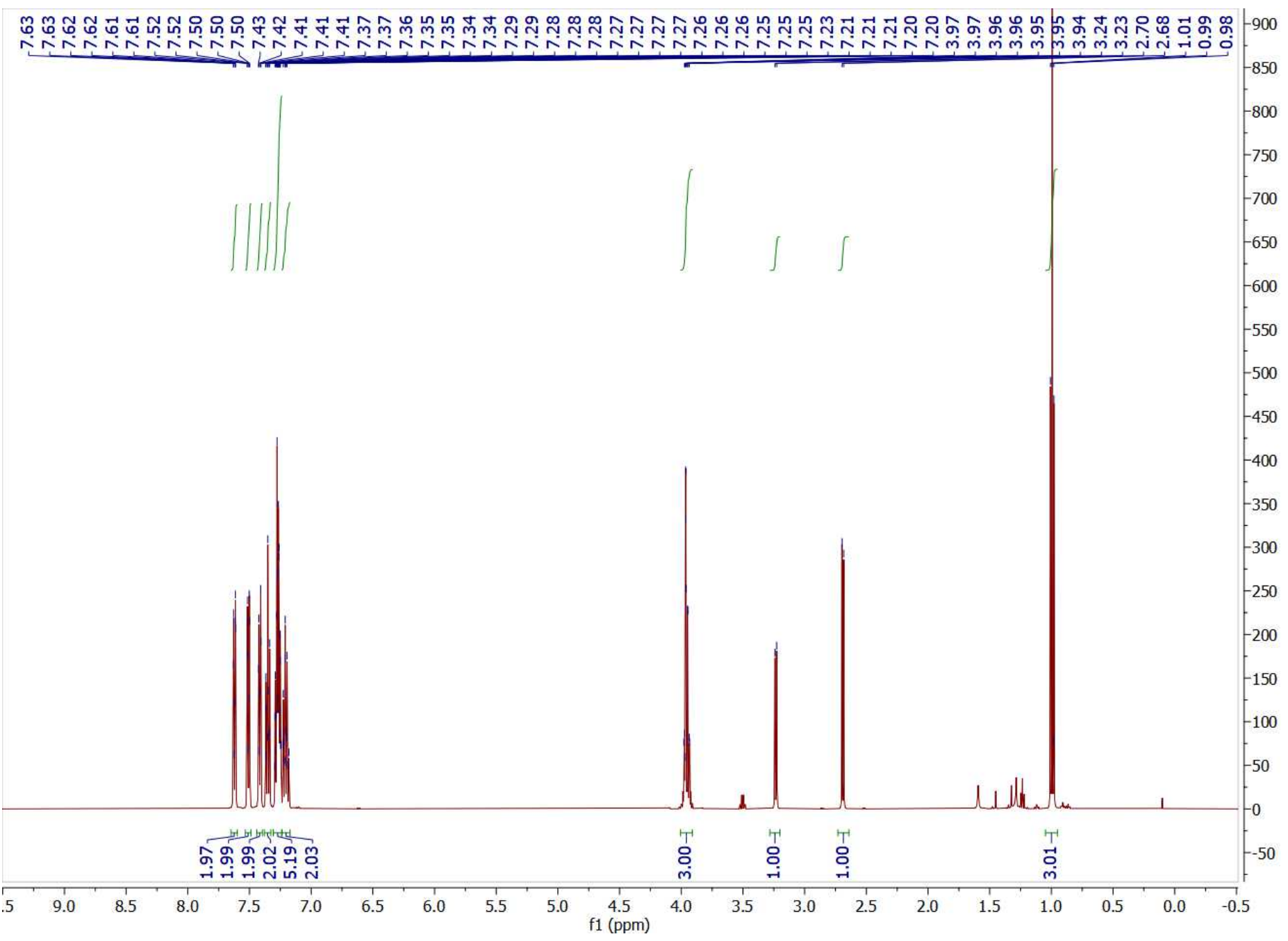




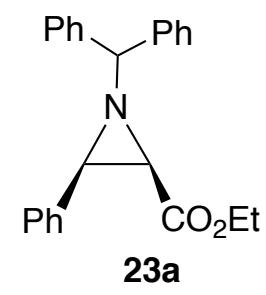

(2R,3R)-ethyl-1-benzhydryl-3-phenylaziridine-2-carboxylate $\mathbf{2 3 a}-{ }^{13} \mathrm{C}\left\{{ }^{1} \mathrm{H}\right\}\left(126 \mathrm{MHz}, \mathrm{CDCl}_{3}\right)$

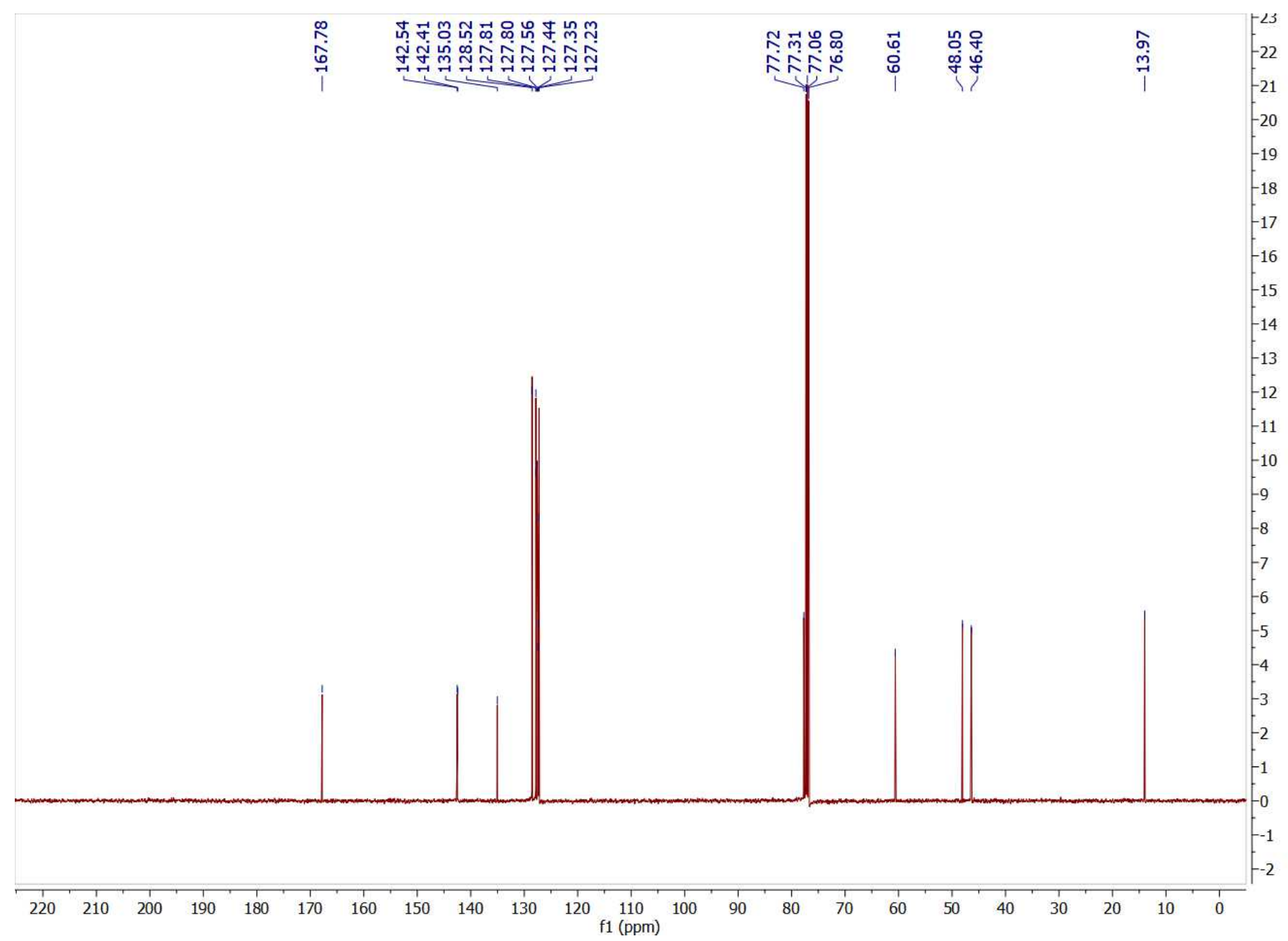




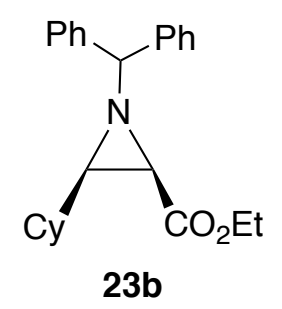

(2R,3R)-ethyl-1-benzhydryl-3-cyclohexylaziridine-2-carboxylate $23 \mathbf{b}-{ }^{1} \mathrm{H}$ NMR $\left(500 \mathrm{MHz}, \mathrm{CDCl}_{3}\right)$

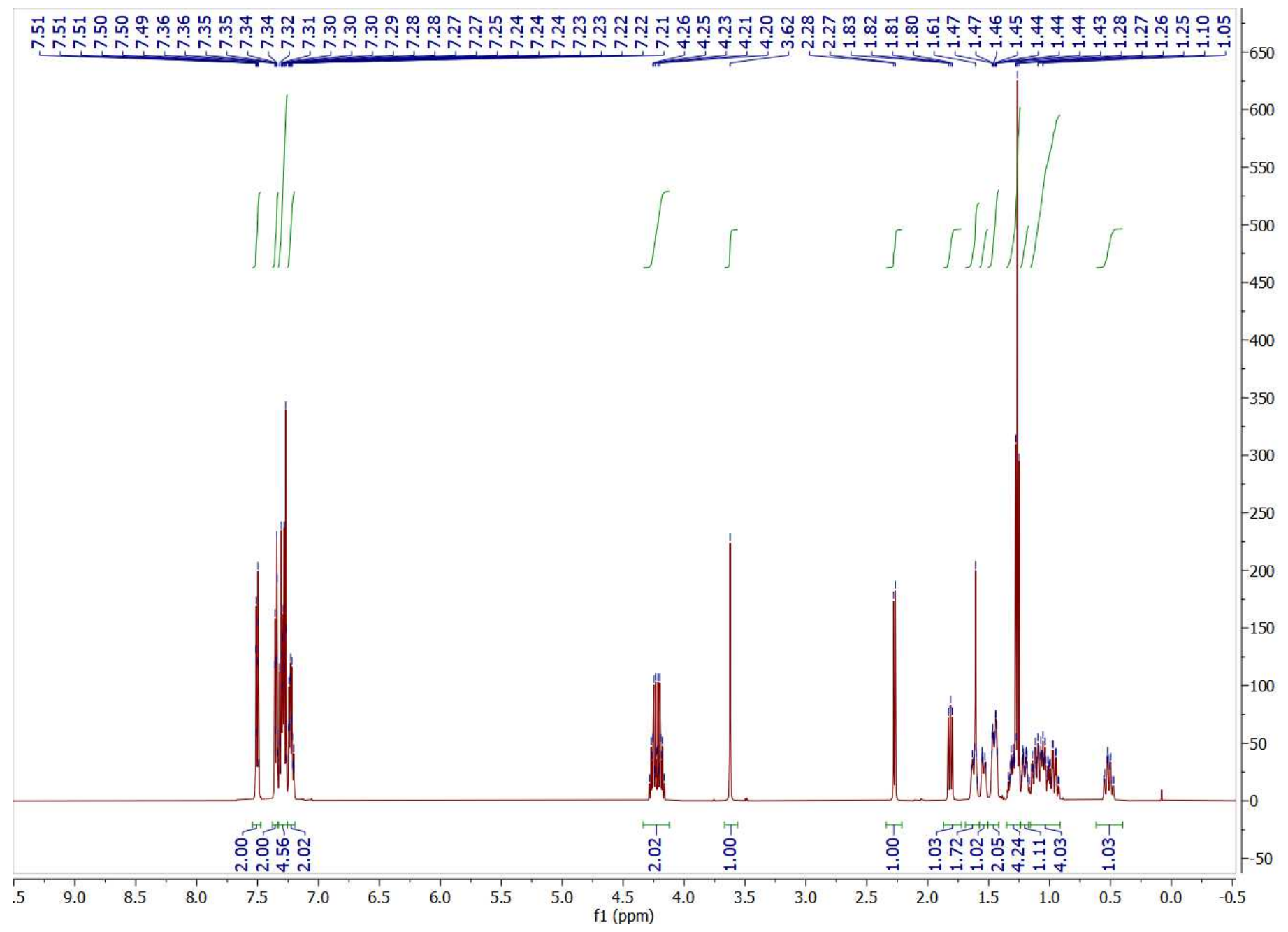




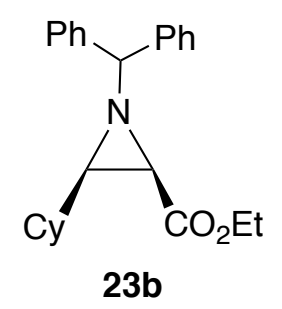

(2R,3R)-ethyl-1-benzhydryl-3-cyclohexylaziridine-2-carboxylate $\mathbf{2 3 b}-{ }^{13} \mathrm{C}\left\{{ }^{1} \mathrm{H}\right\}\left(126 \mathrm{MHz}, \mathrm{CDCl}_{3}\right)$

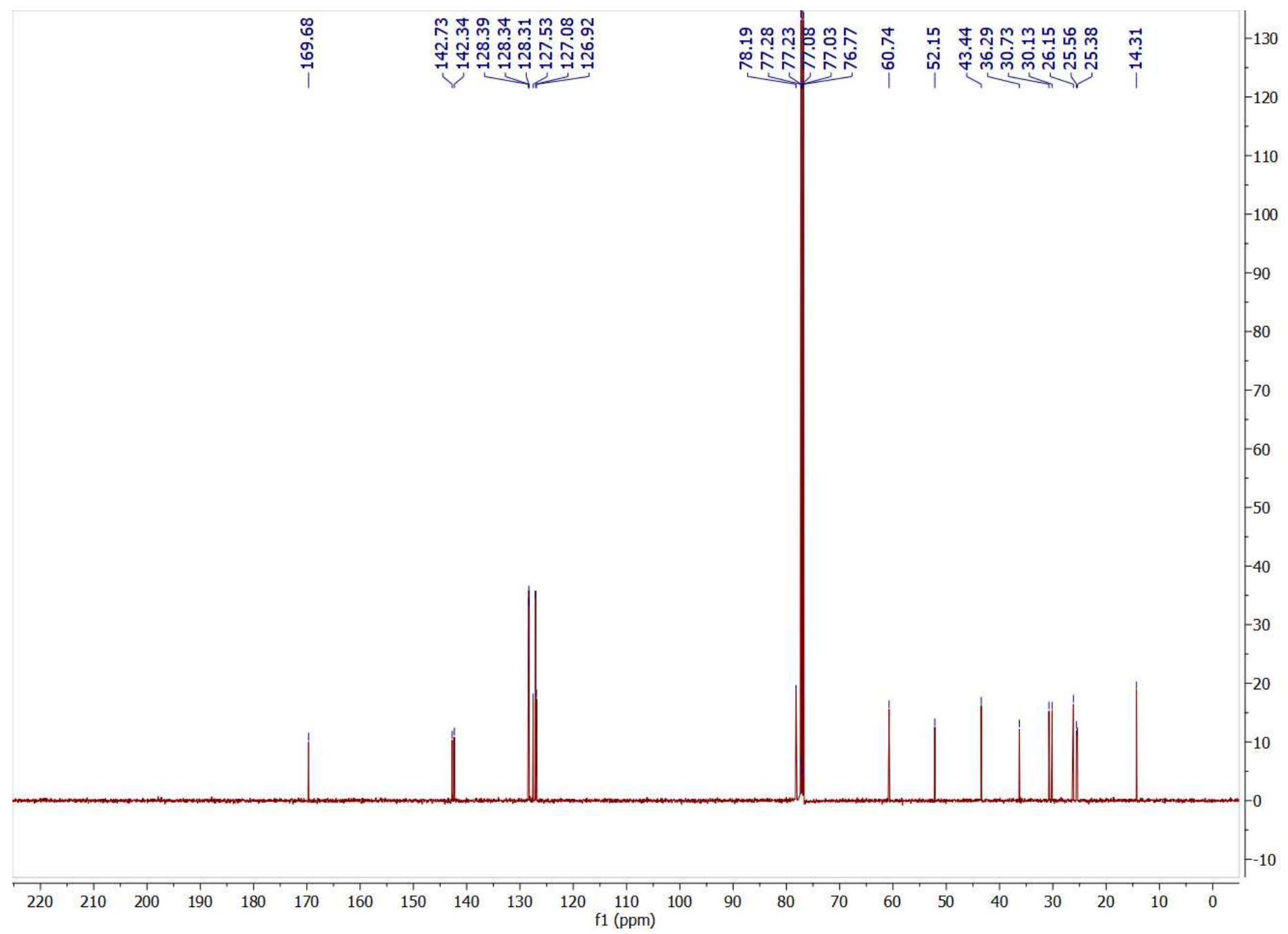



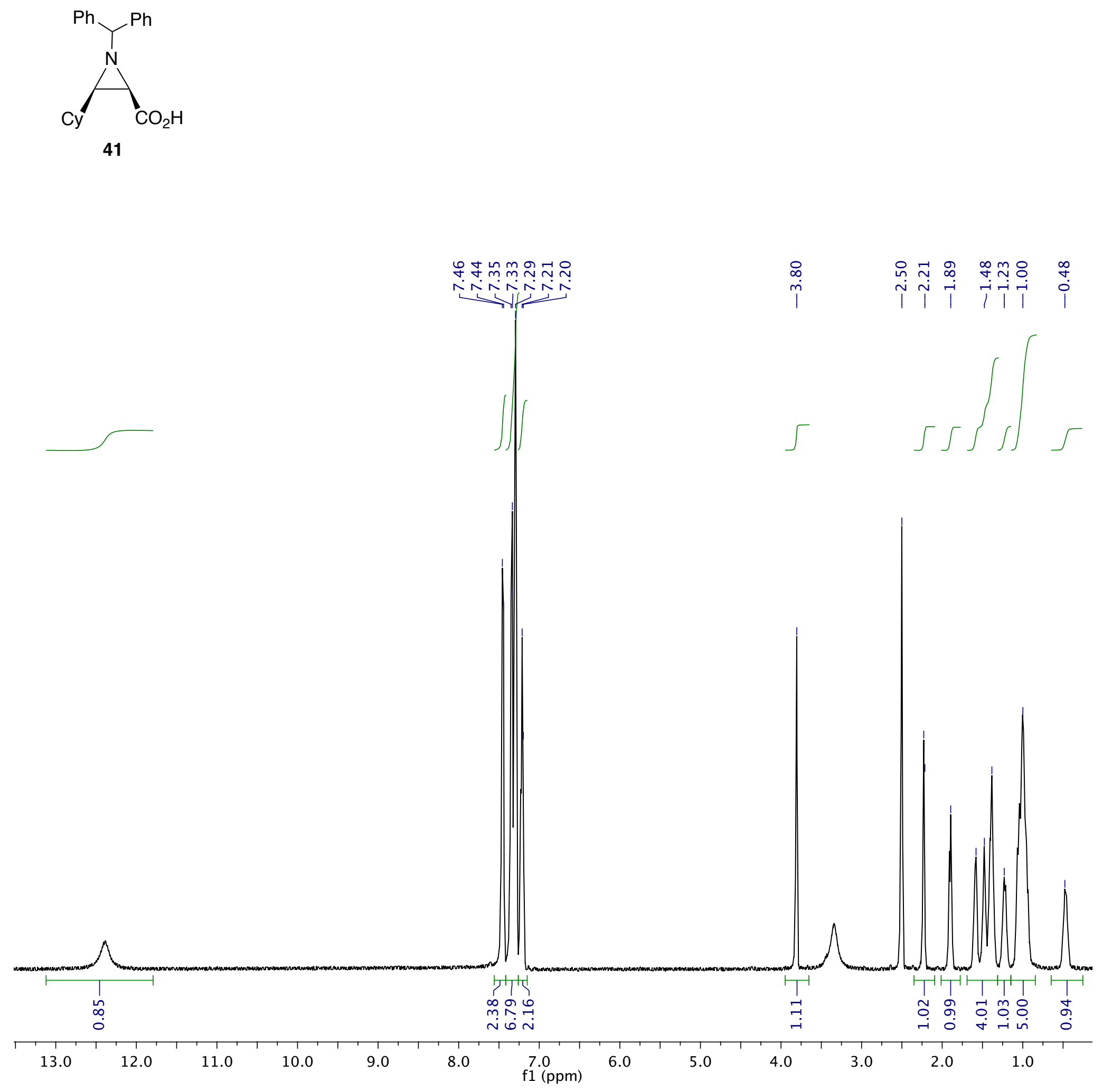

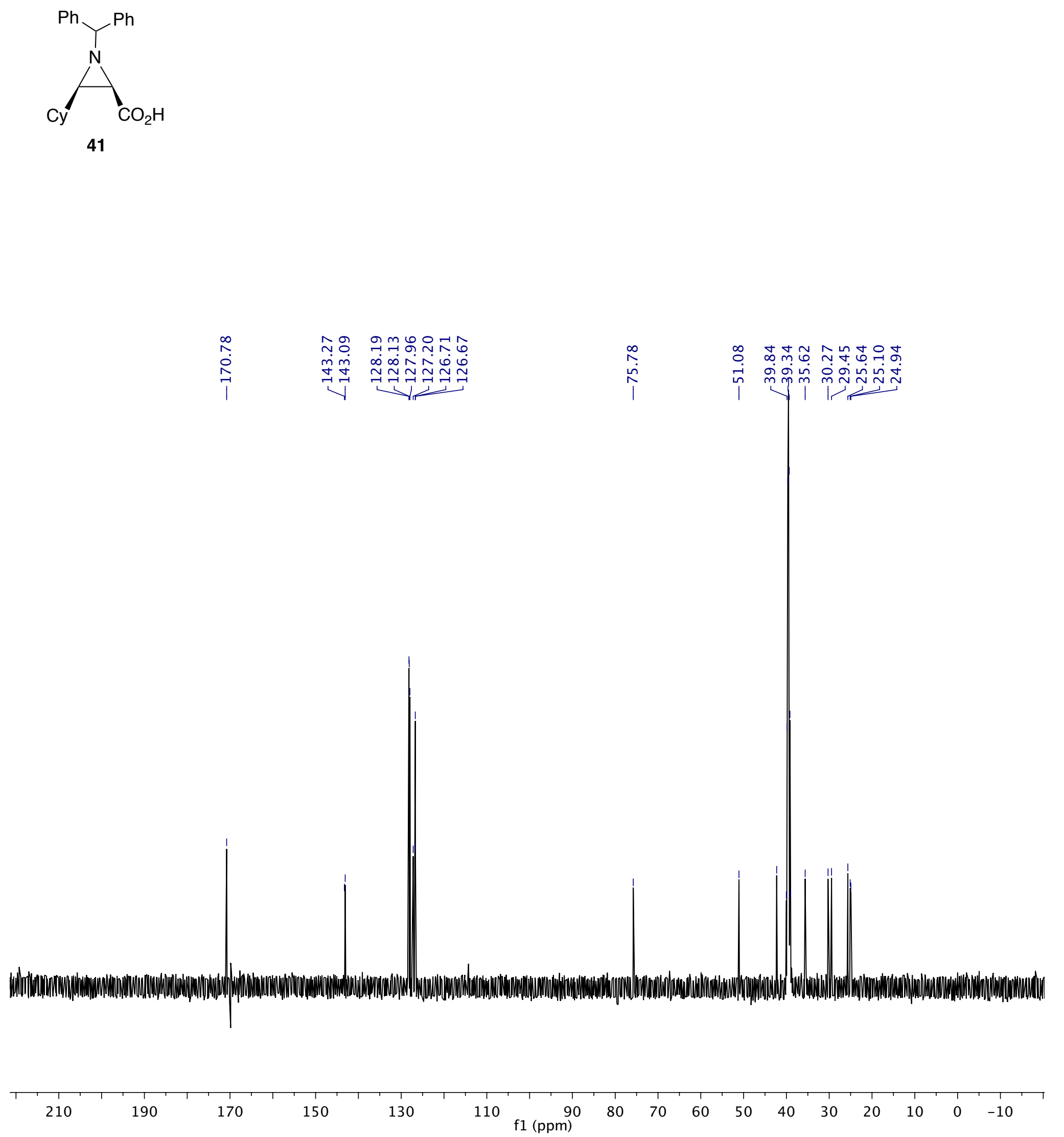